\title{
Subpercent Photometry: Faint DA White Dwarf Spectrophotometric Standards for Astrophysical Observatories
}

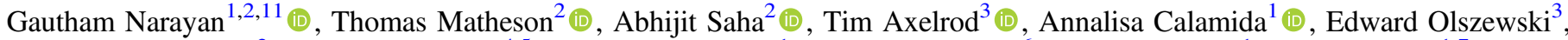 \\ Jenna Claver ${ }^{2}$, Kaisey S. Mandel ${ }^{4,5}$ (1), Ralph C. Bohlin ${ }^{1}$ (10), Jay B. Holberg ${ }^{6}$, Susana Deustua ${ }^{1}$ (1), Armin Rest ${ }^{1,7}$, \\ Christopher W. Stubbs ${ }^{8,9}$ (i), Clare E. Shanahan ${ }^{1}$, Amali L. Vaz ${ }^{3}$, Alfredo Zenteno ${ }^{10}$, Giovanni Strampelli ${ }^{1}$, Ivan Hubeny ${ }^{3}$, \\ Sean Points ${ }^{10}$, Elena Sabbi ${ }^{1}$ (D), and John Mackenty ${ }^{1}$ \\ ${ }^{1}$ Space Telescope Science Institute, 3700 San Martin Drive, Baltimore, MD 21218, USA; gnarayan@ @stsci.edu \\ ${ }^{2}$ National Optical Astronomy Observatory, 950 North Cherry Avenue, Tucson, AZ 85719, USA \\ ${ }^{3}$ The University of Arizona, Steward Observatory, 933 North Cherry Avenue, Tucson, AZ 85719, USA \\ ${ }^{4}$ Institute of Astronomy and Kavli Institute for Cosmology, Madingley Road, Cambridge, CB3 OHA, UK \\ ${ }^{5}$ Statistical Laboratory, DPMMS, University of Cambridge, Wilberforce Road, Cambridge, CB3 OWB, UK \\ ${ }^{6}$ The University of Arizona, Lunar and Planetary Laboratory, 1629 East University Boulevard, Tucson, AZ 85721, USA \\ ${ }^{7}$ Department of Physics and Astronomy, Johns Hopkins University, Baltimore, MD 21218, USA \\ ${ }^{8}$ Harvard University, Department of Physics, 17 Oxford Street, Cambridge, MA 02138, USA \\ ${ }^{9}$ Harvard-Smithsonian Center for Astrophysics, 60 Garden Street, Cambridge, MA 02138, USA \\ ${ }^{10}$ Cerro Tololo Inter-American Observatory, Casilla 603, La Serena, Chile \\ Received 2018 November 29; revised 2019 February 5; accepted 2019 February 6; published 2019 March 20
}

\begin{abstract}
We have established a network of 19 faint (16.5 mag $<V<19$ mag) northern and equatorial DA white dwarfs (WDs) as spectrophotometric standards for present and future wide-field observatories. Our analysis infers spectral energy distribution (SED) models for the stars that are tied to the three CALSPEC primary standards. Our SED models are consistent with panchromatic Hubble Space Telescope photometry to better than 1\%. The excellent agreement between observations and models validates the use of non-LTE DA WD atmospheres extinguished by interstellar dust as accurate spectrophotometric references. Our standards are accessible from both hemispheres and suitable for ground- and space-based observatories covering the ultraviolet to the near-infrared. The high precision of these faint sources makes our network of standards ideally suited for any experiment that has very stringent requirements on flux calibration, such as studies of dark energy using the Large Synoptic Survey Telescope and the Wide-field Infrared Survey Telescope.
\end{abstract}

Key words: cosmology: observations - methods: data analysis - standards - surveys - white dwarfs

\section{Introduction}

The volume of astronomical data has grown by an order of magnitude from the first generation of wide-field surveys, such as the Sloan Digital Sky Survey ${ }^{12}$ (SDSS) and the Two Micron All-Sky Survey ${ }^{13}$ (2MASS), to the second generation of surveys, including Pan-STARRS, ${ }^{14}$ the Dark Energy Survey ${ }^{15}$ (DES), and Gaia. ${ }^{16}$ Together, these surveys have produced terabytes of images and catalogs, measuring the sky from the UV to the near-IR (NIR). While each individual project reports internal relative photometric accuracies of $1 \%-2 \%$, all these surveys must be placed on a single consistent flux scale in order to intercompare across wavelength and redshift.

The most precise existing network of spectrophotometric standard stars for flux calibration, the 93 extrasolar CALSPEC $^{17}$ standards (Bohlin et al. 2014; Bohlin 2014, hereafter B14), has a mean $V=11.1 \mathrm{mag}$, which is close to or brighter than the saturation limit of many of these surveys. While the faint limit of the CALSPEC stars is $V \sim 16 \mathrm{mag}$, most of the objects near this limit are extremely red stars with complex spectral energy distributions (SEDs) that are difficult

\footnotetext{
${ }^{11}$ Lasker Fellow.

12 http://www.sdss.org

13 https://www.ipac.caltech.edu/2mass/

$14 \mathrm{http}: / /$ pan-starrs.ifa.hawaii.edu/public/

15 http://www.darkenergysurvey.org/

16 http://sci.esa.int/gaia/

${ }^{17}$ http://www.stsci.edu/hst/observatory/crds/calspec.html
}

to model. Consequently, surveys have had to resort to establishing networks of secondary or tertiary standards together with synthetic color transformations to tie their natural systems to a common flux scale, such as that defined by the broadband measurements of Landolt (1992). Calibration errors are compounded as the degree of separation from the primary standards increases, and these systematic errors in the photometric calibration propagate into the analyses of several high-precision experiments. The measurement of the equation of state $w$ of dark energy from multisurvey compilations of Type Ia supernovae (SNe Ia) is particularly afflicted by such systematic errors in the photometric calibration (Scolnic et al. 2014). Each of the surveys observes SNe Ia with different telescopes, instruments, and passbands at different sites and targets different mean redshifts. While there are thousands of well-measured $\mathrm{SNe}$ Ia, the constraints on $w$ are dominated by systematic uncertainties.

With the advent of the third generation of wide-field surveys, including the Zwicky Transient Facility ${ }^{18}$ (ZTF) and the Large Synoptic Survey Telescope ${ }^{19}$ (LSST), the volume of observational data will increase by an additional order of magnitude, exacerbating the impact of calibration errors and the mismatch between the standards suitable for calibration and what is practically observable by ground-based facilities. To reduce these systematic errors and obtain less biased measurements of

\footnotetext{
${ }^{18}$ http://ztf.caltech.edu

19 http://www.lsst.org
} 
source fluxes, we need more precise and more accurate photometric calibration. Stubbs \& Brown (2015) review efforts to establish precise astronomical flux calibration and divide these efforts into two categories: (i) approaches where the metrology standard is the SED of an emissive source that can be determined from fundamental physics (e.g., a blackbody spectrum), and (ii) approaches where the metrology standard is based on a detector with known quantum efficiency (QE; e.g., NIST-calibrated Si photodiodes). We employ the first approach in this work.

We have been observing a new all-sky network of faint (16.5 mag $<V<19 \mathrm{mag}$ ) pure-hydrogen (DA) white dwarf (WD) stars with three Hubble Space Telescope (HST) programs (GO-12967 and GO-13711 for northern and equatorial standards, and GO-15113 for southern standards; P.I. Abhijit Saha), and we have obtained multiband above-the-atmosphere photometry to complement ground-based spectroscopy and temporal monitoring. The goal of this work is to use these observations to infer calibrated synthetic SED models, which can then be used as transfer standards by any facility.

Our team presented a proof-of-concept end-to-end analysis in Narayan et al. (2016, hereafter N16) examining the feasibility of modeling the HST observations for 4 of these 23 stars in our Cycle 20 program. N16 assembled a pipeline that largely used existing software packages to process and analyze the data and demonstrated that synthetic DA WD SEDs extinguished by interstellar dust could recover the HST/WFC3 measurements to a few millimagnitudes. Our proof-of-concept analysis also revealed a previously unknown systematic in the photometric zero-points reported by the Mikulski Archive for Space Telescopes (MAST). Even with the limited number of objects available during our pilot study, we were able to detect a systematic difference between the response of the UVIS1 and UVIS2 chips of HST/WFC3. This difference had been masked by the flat-fielding procedure used in the calwf3 pipeline, which produces the processed images available through MAST. This systematic trend has been accounted for in current MAST data products (Deustua et al. 2016, 2017), which improved the flat-fielding procedure and corrected for a systematic bias in the previous calibration, which led to a $4 \%$ underestimate of fluxes on UVIS2.

The exercise revealed the magnitude of the challenge posed by establishing highly accurate spectrophotometric standards: any program aiming to reduce systematic errors arising from photometric calibration must itself be robust against systematic errors arising from photometric calibration. To achieve $1 \%$ level calibration of ground-based surveys, our network of DA WD stars must be calibrated to subpercent accuracy.

This work refines all aspects of the analysis procedure used in N16. In particular, we develop a new methodology to fit our $H S T$ photometry and ground-based spectroscopy simultaneously to infer the underlying DA WD SED. This work produces a calibrated network of northern and equatorial DA WDs with accurately measured SEDs tied directly to the three CALSPEC primary standards, GD 71, GD 153, and G191$\mathrm{B} 2 \mathrm{~B}$. This network of faint spectrophotometric standards is suitable for use by wide-field surveys from the UV to the NIR. Detailed descriptions of the observations and the procedures to obtain and process them are presented in Calamida et al. (2019, hereafter C19), a companion paper to this work.

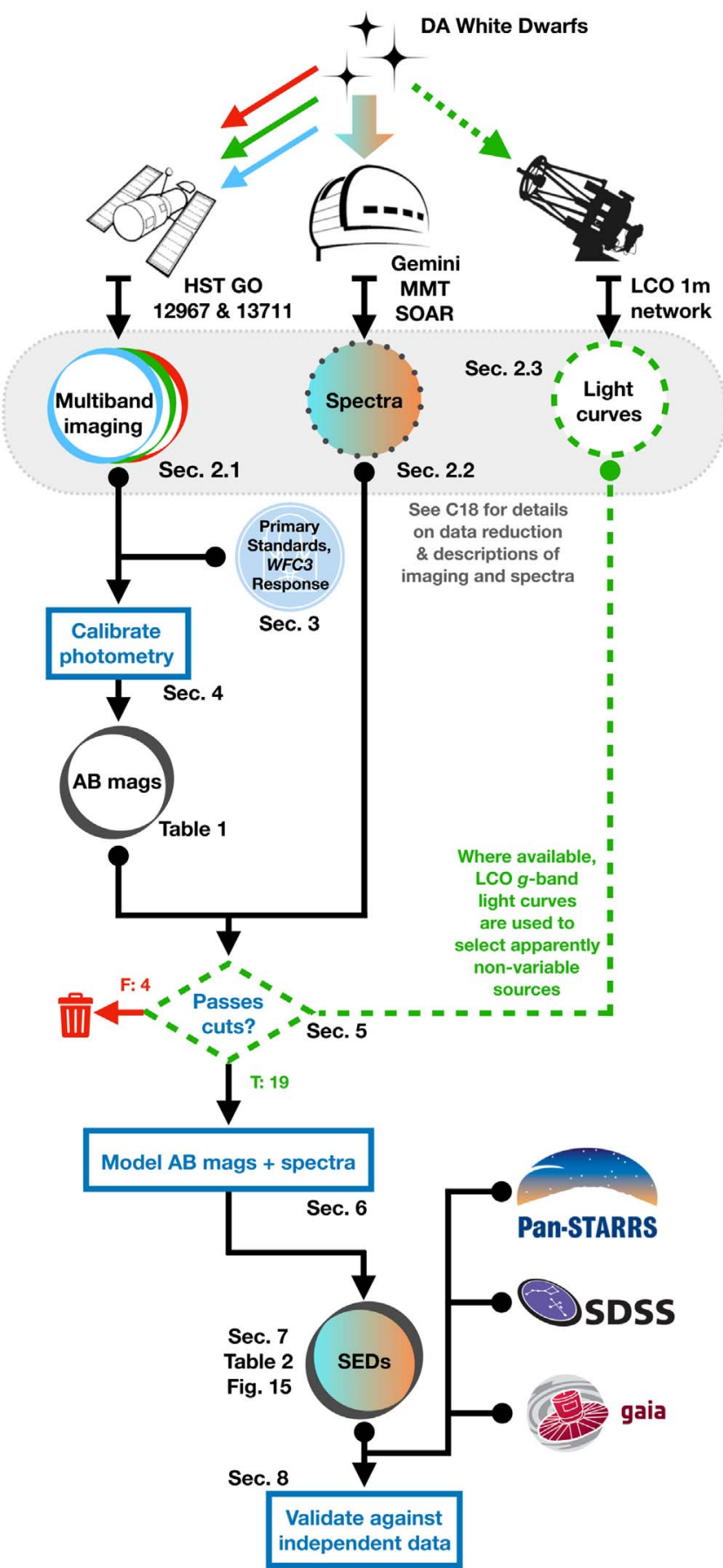

Figure 1. Flowchart of the analysis presented in this work. Data products are indicated by circles, while discrete stages of our analysis are indicated with boxes. Arrows that originate in small dots indicate that the preceding data product is an input to the subsequent analysis. The text labels indicate the sections of this work that correspond to the data product or analysis. We include a brief description of the primary data products produced by our observing program (rounded gray rectangle) that are presented in $\mathrm{C} 19$, a companion paper to this work. Derived data products (outputs from blue rectangles) are available from our archive (see footnote 20 ).

\subsection{Structure of This Paper}

We provide a schematic overview of this work in Figure 1. In Section 2, we provide a brief overview of our observational program, while in Section 3 we highlight the improvements 
made to our procedure from Cycle 20 to Cycle 22. We use a Bayesian hierarchical model to combine our multicycle $H S T$ observations and to generate a combined photometric catalog that serves as the primary input for our analyses; we describe this model in Section 4. In Section 5 we impose selection cuts to eliminate objects that do not meet the stringent requirements of our program. We develop a Bayesian model for a joint analysis of the spectroscopic and photometric measurements to infer SEDs for our DA WDs in Section 6. The results of our analysis of the data are presented in Section 7, together with a detailed examination of potential sources of systematic error. We test that the SED models are consistent, both internally and with constraints from external data sets, in Section 8. Finally, in Section 9, we summarize our analysis and outline our plan to complete this program. Tables of photometric and spectroscopic observations, as well tables of inferred model parameters, photometric residuals, and the final calibrated SEDs, are available through our archive. ${ }^{20}$

\section{Design of the Observing Program}

Our observational program consists of three components: (i) multiband HST imaging using WFC3, (ii) ground-based spectroscopy using a variety of low-dispersion long-slit spectrographs on large-aperture telescopes, and (iii) temporal monitoring using the Las Cumbres Observatory (LCO) network of robotic $1 \mathrm{~m}$ telescopes. All three components are critical to our program. Here we provide an overview of the different observations, focusing on why each component is necessary for our analysis. A detailed description of what observations were obtained and how the data are processed is provided in C19. This work focuses on the analysis of these observations.

\subsection{HST Photometry}

The key observational component that distinguishes our program from other efforts to establish spectrophotometric standards, such as Allende Prieto et al. (2009), is our panchromatic $H S T /$ WFC3 photometry: we can set the flux scale for each object from the UV to the NIR without systematic effects from atmospheric extinction. Ground-based programs to establish spectrophotometric standards face inherent limitations:

1. The transmissivity of the atmosphere in the UV and NIR makes sufficiently high signal-to-noise ratio $(\mathrm{S} / \mathrm{N})$ ground-based observations of faint standards prohibitive.

2. There are significant gray and chromatic variations (arising from aerosol scattering, Rayleigh scattering, and oxygen, ozone, and precipitable water vapor absorption) on short angular and temporal scales in the optical.

3. Many stars with a long legacy of spectrophotometric measurements that are employed as standards, such as Vega and $\mathrm{BD}+17^{\circ} 4708$, are not ideal reference calibrators and have complex, hard-to-model SEDs, or exhibit measurable variability. Most of the remaining well-measured spectrophotometric standards that could be used as a reference for our new standards also saturate the instruments on modern large-aperture facilities-one of the motivations for establishing our network of faint standards in the first place.

\footnotetext{
${ }^{20}$ https://github.com/gnarayan/WDdata
}

These effects have limited the absolute flux calibration accuracy of ground-based surveys to the few percent level (Stubbs \& Tonry 2006). The resulting calibration errors are one of the leading systematic effects afflicting dark energy studies with $\mathrm{SNe}$ Ia that rely on a comparison of the brightness of distant $\mathrm{SNe}$ with nearby $\mathrm{SNe}$, often observed by a completely different survey (Scolnic et al. 2014).

To overcome these limitations, we obtained photometry using $H S T$, avoiding the time-variable atmosphere of the Earth. This choice has various ancillary benefits. The HST/WFC3 throughput was precisely determined pre-flight, and staff at the Space Telescope Science Institute (STScI) have continually monitored the system to tie WFC3 measurements to other HST instruments, particularly the Space Telescope Imaging Spectrograph (STIS) and the Advanced Camera for Surveys (ACS).

Our use of HST makes the three CALSPEC primary standards, GD 71, GD 153, and G191-B2B, the natural choice for our network's spectrophotometric reference. These standards have the advantage of being the same class of astrophysical source as our targets. Moreover, all have an extensive set of observations with a wide variety of instruments; B14 used these observations to constrain their line-of-sight interstellar extinction to be consistent with $<2$ mmag in the optical.

While planning our HST/WFC3 observations, we avoided sources with a detectable close companion in archival SDSS and Pan-STARRS images and selected objects with low lineof-sight Galactic reddening by limiting the color excess to $E$ $(B-V)<0.2 \mathrm{mag}$. We obtained observations in $\mathrm{F} 275 \mathrm{~W}$, F336W, F475W, F625W, F775W, and F160W to establish the flux scale and constrain any interstellar extinction. Given the median exposure times of our observations, stacking both individual exposures taken for cosmic-ray rejection yields $5 \sigma$ limiting magnitudes in $\{\mathrm{F} 275 \mathrm{~W}, \mathrm{~F} 336 \mathrm{~W}, \mathrm{~F} 475 \mathrm{~W}, \mathrm{~F} 625 \mathrm{~W}$, F775W, F160W $\}$ of $\{22.8,23.1,24.1,24.3,24.6,25.4\} \mathrm{AB}$ mag. These deep observations are sufficient to rule out $\mathrm{O}-\mathrm{K}$ star companions. The presence of such companions was strongly disfavored from inspection of SDSS and PanSTARRS images, but our HST observations extend further into the UV and the IR and are deeper than these ground-based surveys. We discuss our selection cuts in detail in Section 5 and eliminate one object, SDSS J041053, from our sample. This object is now known to be a DA+M:E binary system (Kleinman et al. 2013), and we find that it exhibits significantly different colors from the other targets in our sample. This is consistent with Eisenstein et al. (2006), which demonstrates that the temperature inferred from the spectra of these DAsubdwarf $M$ binary systems have a significantly different correlation with SDSS colors than isolated DA WDs, and they form a different locus in SDSS color-color diagrams.

Our NIR observations are critical to establishing our DA WDs as useful spectrophotometric standards for major future space-based observatories, including the James Webb Space Telescope and Wide-Field Infrared Survey Telescope (WFIRST). The utility of these standards is not limited to space-based observatories. By establishing our network of faint standards directly on the CALSPEC WD scale, we extend the $H S T$ heritage of precision calibration to all current and future observatories.

\subsection{Ground-based Spectroscopy}

While the HST photometry is sufficient to establish our targets as standards in our program passbands, we wish to 
establish spectrophotometric standards that can be used by any ground- and spaced-based facilities. This requires that we construct an SED model for our targets, which in turn requires that we infer any intrinsic and extrinsic properties of standards that correlate with their flux. Fully radiative pure-hydrogen DA WD models have been used to establish SEDs for over three decades (see Section 4 of Bohlin et al. 2014, and references therein). Only two parameters are necessary to model their SED: temperature $T_{\text {eff }}$ and surface gravity $\log g$. The second component of our observational program is to obtain a high-S/N $(>20)$ spectrum of our targets using large-aperture ground-based facilities to constrain these two parameters.

The depths of the hydrogen Balmer lines of DA WD stars are strongly correlated with $T_{\text {eff }}$, while the line widths are very sensitive to $\log g$, although all line features depend on both parameters, and the line shapes are impacted by nonideal effects arising from proton and electron perturbations (Tremblay \& Bergeron 2009). The shape of the DA WD continuum is largely determined by $T_{\text {eff }}$ and interstellar reddening. Accounting for reddening is critical for unbiased inference of the intrinsic SED parameters. Our requirement for faint targets implies that our DA WDs will be significantly more distant than the CALSPEC primary standards and therefore subject to extinction. Within our Galaxy, the interstellar reddening can be modeled by extinction curves such as those of Fitzpatrick (1999, hereafter F99) and O'Donnell (1994). Given these curves, the extinction at any wavelength can be described by two parameters: the extinction in the $V$ band $A_{V}$, and the ratio of $A_{V}$ and the color excess $E(B-V)$, denoted by $R_{V}$.

The intrinsic $T_{\text {eff }}$ model parameter and extrinsic $A_{V}$ model parameter are strongly inversely correlated, as lowering the temperature and increasing the line-of-sight extinction both lead to a redder continuum. While it is in principle possible to constrain all these model parameters solely from multiband photometry, the photometry can only weakly constrain the surface gravity, and the number of independent multiband observations spanning a range in wavelength long enough to break the degeneracy between temperature and reddening makes a purely photometric analysis impractical. Spectroscopy is critical to disentangling these distinct physical processes, as they affect the Balmer line shapes differently, and care must be taken in obtaining and reducing these observations.

The wide hydrogen Balmer features and faintness of our targets make low-dispersion $(R \sim 300-1000 \mathrm{lp} / \mathrm{mm})$, long-slit instruments the optimal solution for our program's spectroscopic requirements. As our flux scale is independently set by the HST photometry of the CALSPEC primary standards, we use relatively narrow slits compared to most spectrophotometric efforts in order to reduce the sky background and to ensure that the instrumental resolution is smaller than the Balmer line widths. We employ the optimal extraction algorithm of Horne (1986) to extract the 1D trace from the 2D spectrum images and recover the flux at both the blue, where the drop in the detector QE dominates the increase in the DA WD flux, and the red, where both the QE and WD flux drop precipitously, while the amplitude of fringing increases. The narrow slit width also makes us relatively insensitive to small centering errors that would propagate into velocity shifts. We remove cosmic rays and use telluric corrections determined from observations of two standards with a smooth blue and red continuum, respectively, to remove the atmospheric absorption lines from ozone and water vapor. We illustrate one spectra $\mathcal{S}$ for each of our targets in Figure 2.

Throughout this work, variables representing a spectral flux density are denoted with a subscript $\lambda$ when reported per unit vacuum wavelength and with a subscript $\nu$ when reported per unit vacuum frequency. The equations in Section 4.3 can be used to transform between the two conventions. The data products provided with this work are reported as $F_{\lambda}$.

The spectroscopy, without any additional photometric constraints, can be used to determine $T_{\text {eff }}$ and $\log g$ to a few percent. This inference is sufficient to impose weak selection cuts. We select objects with $T_{\text {eff }}>20,000 \mathrm{~K}$ to ensure that their atmospheres are fully radiative. Theory suggests that the WD luminosity in the $T_{\text {eff }}>20,000 \mathrm{~K}$ regime is too high for circumstellar dust grains to survive (Koester et al. 2014).

Even though we have only a few distinct epochs of spectroscopy, these observations do place limits on the variability of our sources. Our spectra do not exhibit any absorption bands to indicate the presence of $\mathrm{M}$ dwarf or subdwarf companions such as those of Silvestri et al. (2006). At least $10 \%$ of WDs exhibit magnetic fields of $10^{6}-10^{9} \mathrm{G}$ level (Liebert et al. 2003). Our spectroscopic observations allow us to rule out targets exhibiting strong magnetic fields, which would be apparent in Zeeman splitting of the Balmer lines, or in the presence of the Minkowski bands-shallow and broad absorption bands near 3650, 4135, and $4466 \AA$. WDs with weaker magnetic fields cannot be excluded on the basis of the spectroscopy alone, as the high surface gravity broadens absorption lines, making Zeeman splitting undetectable without high-resolution observations.

Our analysis in Section 6 describes the WD with a non-LTE (NLTE) pure-hydrogen model atmosphere, and we look for deviations from this assumption. Our observation methodology and our extraction procedures optimize the $\mathrm{S} / \mathrm{N}$ of any narrow nonhydrogen features such as the $\mathrm{Ca}$ II doublet and $\mathrm{MgN}$ species, occasionally seen in DA WD spectra (see, e.g., Farihi et al. 2012). While these features have largely been observed in objects cooler than our temperature cutoff, the fraction of hot DA WDs exhibiting such features is only weakly constrained by existing observations. Metal lines have been seen in highresolution $\left(R=2.3 \times 10^{5}\right) \mathrm{UV}$ spectra of G191-B2B (Bohlin et al. 2014), but the number fraction of elements relative to hydrogen is $\lesssim 10^{-6}$ (inferred abundances for identified species are given in Table 8 of Rauch et al. 2013). The presence of these trace metal lines makes a $<2$ mmag difference in F275W relative to a pure-hydrogen model with the same extinction as the Bohlin et al. (2014) model. Metal lines have not been identified in high-resolution spectra of either GD 71 or GD 153.

Following this initial analysis, we combine our spectroscopy and photometry to infer the SED for each DA WD. While we use the shape of the continuum for inference, the absolute normalization of the ground-based spectroscopy is typically only accurate to a few percent and is often tied to spectrophotometric standards that are less precisely established than our target DA WDs. The noise model we employ in Section 6.7 can account for broad correlated errors in the continuum shape and prevents biases in the inferred intrinsic parameters.

\subsection{Temporal Monitoring}

The final component of our observing program is designed to detect any photometric variability exhibited by our DA WDs. This includes extrinsic variability (binary companions or debris 


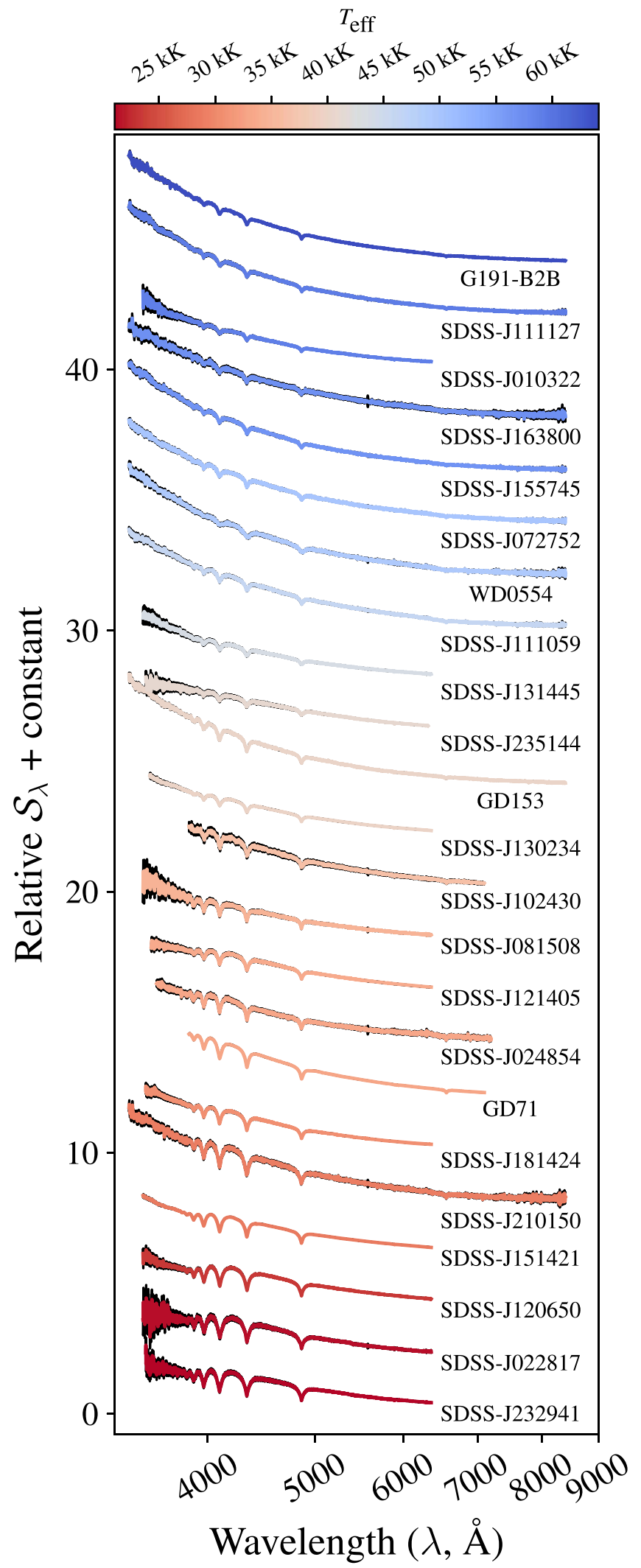

Figure 2. Sequence of observed spectra $\mathcal{S}_{\lambda}$ of our DA WDs, ordered by inferred $T_{\text {eff }}$ (indicated by color, with uncertainties on the flux shown in the black shaded region about each trace). The depths of the hydrogen Balmer lines are sensitive to the temperature of the photosphere, while the line widths are sensitive to $\log g$. The shape of the continuum is sensitive to temperature and interstellar reddening and can exhibit correlated errors with wavelength arising from errors in flux calibration of the spectrum. disks) and weak intrinsic variability over short and long timescales (stochastic outbursts or pulsation). We are obtaining repeated observations of our targets using the Las Cumbres Network of robotic $1 \mathrm{~m}$ telescopes. These time-series data allow us to look for variability over timescales of a few hours to several months. We also look for evidence of variability in archival data from other ground-based surveys, such as the Pan-STARRS1 $3 \pi$ Survey. This program is still in progress, and not all of DA WDs have sufficient observations ( $\gtrsim 35$ epochs with approximately logarithmic spacing) to exclude variability comprehensively at present. C19 present results from our temporal monitoring to date. Based on the current observations, we exclude WD 0554 and SDSS J203722 as variable, as described in Section 5. More detailed results from our temporal monitoring will be presented in future analysis, and we will continue to obtain observations of our targets to rule out variability on timescales longer than a year.

Despite this component of our observing program not being complete, there are several reasons to expect the fraction of objects in our network that exhibit variability to be small $(<2 \%)$. Our HST photometry and spectroscopy are sufficient to exclude main-sequence stars and subdwarf companions. Our selection criteria also exclude cool DA WDs in the ZZ Ceti instability strip (Gianninas et al. 2014), which have convective atmospheres and may exhibit strong variability on timescales as short as a few minutes (Winget \& Kepler 2008). Our spectroscopy excludes strongly magnetic white dwarfs (MWDs), which have largeamplitude photometric variations on timescales of a few hours to a few days (see, e.g., Brinkworth et al. 2013).

Studies of nearby WDs can be used to place limits on the expected fraction of objects that exhibit intrinsic variability or have binary companions. Hermes et al. (2017, hereafter H17) have carried out a detailed analysis of variability for a sample of 398 high-probability WDs (252 spectroscopically confirmed, 146 photometrically selected on the basis of their SDSS colors) having Kepler $K_{\mathrm{P}}<19 \mathrm{mag}$. The majority of these objects are DA, but the sample also includes helium-dominated DB and DO stars, carbon-dominated DQ stars, and continuum-dominated DC stars. Using the Kepler/K2 30-minute cadence light curves of these sources, H17 identified only nine that exhibit variability exceeding a peak-to-peak amplitude of $1 \%$ on $1 \mathrm{hr}$ to 10-day timescales, i.e., $>97 \%$ of their sample of apparently isolated and nonpulsating DA WDs do indeed make good flux standards. The controls adopted for this work likely reduce the percentage of variables even further. Of the nine sources that $\mathrm{H} 17$ found to exhibit variability, four variable sources were photometrically selected and have colors consistent with $T_{\text {eff }}<9000 \mathrm{~K}$, while an additional two spectroscopic targets have $T_{\text {eff }} \sim 100,000 \mathrm{~K}$ and significantly outshine their putative companions. These six objects would have been excluded from our analysis by the selection cuts imposed in Section 2.2. An additional 15 of the 398 sources exhibit strong magnetic fields and would have been excluded on the basis of their spectroscopy. The H17 analysis suggests that the fraction of objects that exhibit intrinsic variability is likely less than $2 \%$ of the sample.

Toonen et al. (2017, hereafter T17) have modeled the evolution of a nearly complete WD sample within $20 \mathrm{pc}$ with a binary population synthesis approach. Beginning with an initial binary fraction of $50 \%$, their population synthesis model suggests that the most common outcome for the system is to 
evolve through Roche lobe overflow, followed by a common envelope phase and a merger, with $\sim 65 \%-80 \%$ of events ending as isolated sources. This conclusion is in good agreement with the observed $78 \%$ fraction of isolated WDs in the local population. The majority of the remaining $20 \%-35 \%$ of WDs are in wide binaries. Even after factoring in the greater distance to our network of DA WDs, which is between 10 and 50 times farther than the local population, these resolved companions would be easily detected in our 0 ". 04 pixel $^{-1}$ resolution of our UVIS images. We must also consider the possibility of an unresolved companion that contaminates the flux. T17 expect only $0.5 \%-1 \%$ of the local population to consist of unresolved WD-MS companions, which agrees well with the observed fraction of $0.4 \%$ in the local population. Of this small fraction, only late-type companions are of concern, as emission from other stars should be readily evident in our optical spectra and our multicolor photometry.

\section{Improvements Made in the Design of the Observing Program}

The proof-of-concept N16 analysis provided us with several valuable insights into how to reduce various sources of systematic bias that could impact our HST photometry and ground-based spectroscopy programs. We used these insights to improve our methodology for our Cycle 22 and Cycle 25 programs. We provide a brief summary of the changes to our methodology below, focusing on how these improvements affect our analysis in Section 6 . The changes discussed here have been ordered by our assessment of their impact on the output SEDs that are the result of our forward-modeling of the observations. While the differences caused by some of these changes taken individually are small, we emphasize that their effects are cumulative.

\subsection{Addition of F275W Observations to Constrain Extinction Due to Interstellar Dust}

In Cycle 20, we obtained photometry in F336W, F475W, F625W, F775W, and F160W. In N16, we modeled the photometry and spectroscopy independently, using the spectroscopy to constrain $T_{\text {eff }}$ and $\log g$ and define the unreddened DA WD model atmosphere. We ascribed the difference between the observed photometry and the unreddened synthetic photometry of the model photometry to extinction due to diffuse interstellar dust with $R_{V}=3.1$. In Cycle 22 we added observations in F275W to improve our ability to determine $A_{V}$. While $\mathrm{F} 275 \mathrm{~W}$ does not extend sufficiently far into the UV to cover the $2100 \AA$ bump in the reddening law and strongly constrain $R_{V}$, it increases the lever arm in wavelength significantly, reducing the correlation between the inferred surface temperature of the WD and the reddening. The response curves of the passbands ${ }^{21}$ used in our program (Deustua 2016), the CALSPEC SED of GD 153, and the F99 transmission for $E(B-V)=0.2 \mathrm{mag}$ are illustrated in Figure 3.

\subsection{Additional Observations to Exclude Cosmic Rays}

Our exposure times were often hundreds of seconds, even in the bluest bands, where our faint DA WDs are brightest

\footnotetext{
${ }^{21}$ The specific pysynphot reference files used in this work are listed in https://github.com/gnarayan/WDdata/blob/master/photometry/synphot_ obscomponents.txt and included with the data. They match the master reference for HST/WFC3 maintained at http://www.stsci.edu/hst/observatory/crds/ SIfileInfo/pysynphottables/current_wfc3_throughput_html.
}

intrinsically, in order to achieve the requisite $\mathrm{S} / \mathrm{N}$ for this program. Given these long exposure times and the rate of cosmic-ray events, we split our Cycle 20 exposures up into at least two, but typically three, repeat exposures per passband to avoid contamination. While fully mitigating cosmic rays requires an even higher number of repeats, we were constrained by the need to avoid overflowing the WFC3 data buffer before data downlink and to fit a large number of observations for a large number of targets into a modest number of orbits.

While the low number of repeat exposures proved sufficient for most sources, N16 found that the flux in the bias-corrected, flattened "flt" images corrected by the pixel area map (PAM) is inconsistent in some of the cases with only two repeats. In these cases, it was not possible to determine what the true flux of the source was. In Cycle 22, we ensured that we obtained at least one additional exposure for any observations in Cycle 20 with only two repeats, dithering exposures slightly to aid in cosmic-ray and hot-pixel rejection and mitigate the effect of bad pixels. In principle, the process also allows better sampling of the point-spread function (PSF), but given the limited number of exposures for image combination, this gain in resolution is not realized.

\subsection{Intracycle Monitoring of CALSPEC Primary Standards}

In the N16 analysis we used the HST/WFC3 zero-points reported by MAST but discovered that there were unmodeled systematic differences between the UVIS1 and UVIS2 chips of WFC3 that we had to correct for. We decided to mitigate this systematic by observing the three CALSPEC primary standards over the duration of Cycle 22 in all our program passbands, roughly contemporaneously with observations of our science targets. This allows us to estimate the zero-point in each passband and tie our faint DA WDs directly to the CALSPEC primary standards. As exposure times of the bright primary standards are short (less than $1 \mathrm{~s}$ in some passbands), we follow a recommendation from Sahu et al. (2014) and use shutter blade "A" on the WFC3 UVIS channel, rather than the default of alternating between blades " $\mathrm{A}$ " and " $\mathrm{B}$ " for consecutive exposures. This reduces shutter-induced vibrations and results in a $\sim 10 \%$ narrower PSF compared to our Cycle 20 observations (Hartig 2008).

\subsection{Use of Primary Imaging Data Products to Avoid calwf3 Pipeline Systematics}

In N16, we used the combined "drz" images produced by the multidrizzle package and available through MAST as inputs to the SourceExtractor photometry routine (Bertin \& Arnouts 1996). Measuring the flux solely from the drz images missed instances where the flux measurements from the individual PAM-corrected flt images were in disagreement. Moreover, while the multidrizzle algorithm is designed to conserve flux, the growth curves of some point sources differed significantly from the median growth curve for all point sources on the drz image, but this behavior was not exhibited by the corresponding sources on the individual PAM-corrected flt images.

For the Cycle 22 data presented in C19, we reprocessed individual exposures through the calwf 3 pipeline, disabling the default auto-scaling of UVIS2 flux levels to match UVIS1. We applied a correction for charge transfer efficiency (CTE) losses to the flt images, producing "flc" images, which we 


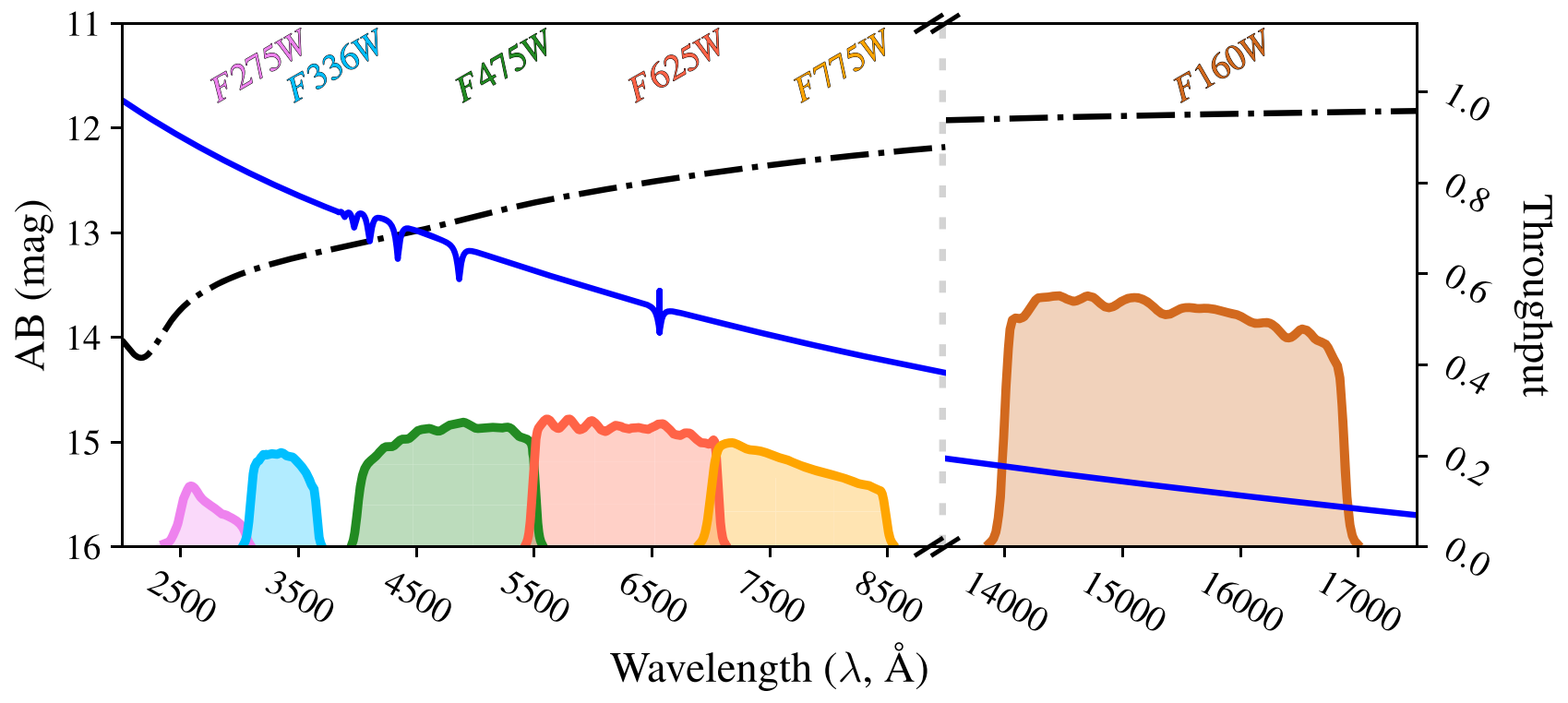

Figure 3. Throughput of the $H S T$ /WFC3 passbands ( $y$-axis on right) used in our program, shown together with the SED of CALSPEC primary standard GD 153 in $\mathrm{AB}$ mag (blue, $y$-axis on left). The F99 transmission (dot-dashed black, $y$-axis on right) for $E(B-V)=0.2$ with the canonical Milky Way $R_{V}=3.1$ is also shown, as we expect our network of DA WDs to be affected by dust along the line of sight.

photometered directly, bypassing multidrizzle entirely. Another advantage to this approach is that it avoids combining data taken at very different times during the same cycle directly, allowing us to account for any small changes in the system throughput over the duration of the cycle, by contemporaneously observing the three primary CALSPEC standards, GD 71, GD 153, and G191-B2B, at the same detector location as our standards. A simple measurement of the relative count rate is necessary to determine apparent magnitudes, thereby avoiding many potential sources of systematic error.

Using the primary data products does incur a cost: while the drz images created by multidrizzle undergo cosmic-ray and hot-pixel rejection, our flc images do not. We attempted to apply the cosmic-ray rejection algorithm directly to the flc images but found that this process could change the shape of the PSF, particularly in F625W and F775W, where point sources are mistakenly classified as cosmic rays, due to the narrow FWHM. As the fraction of measurements of our program stars likely to be affected by cosmic rays is small, we elected not to apply any cosmic-ray rejection to the images and instead accounted for outliers in our photometric catalogs in Section 4.2. The drz image combines the multiple dithers to subsample the PSF and improve the resolution across the field of view, but as noted previously, this gain is not realized given their limited number. It may be possible to adjust the numerous settings of the multidrizzle algorithm to eliminate instances where magnitudes measured from the drz images and the individual cosmic-ray- and hot-pixel-rejected " $\mathrm{Cr} j$ " images disagree. For this work, we prefer to work directly with less processed data products presented in $\mathrm{C} 19$ in order to avoid introducing systematic errors in the image processing that cannot easily be tracked back to a single exposure.

\subsection{Optimization of Imaging to Reduce Detector Systematics and Improve Program Efficiency}

In our Cycle 20 observations the primary target was placed at the center of the UVIS1 chip, and we applied a post-flash of
12 electrons to increase the background. CTE trails were visible on the drz images, and N16 accounted for these trails by selecting a large enough aperture for photometry to include the majority of the flux. The target was placed at the center of the IR chip for observations in F160W.

For the data obtained during Cycle 22, we elected to place our target in the UVIS2 readout corner, on the "C" amplifier, to minimize the total number of pixels over which charge has to be transferred before reaching the readout registers and the A/D converter. While minimizing CTE losses benefits observations in all UVIS passbands, this choice particularly benefits observations in F275W, as the response of UVIS2 is $30 \%$ higher than that of UVIS1 for wavelengths blueward of $3000 \AA$. Furthermore, ghosting on the " $\mathrm{C}$ " amplifier is lower than on the "A" and "D" amplifiers of the WFC3 UVIS channel. We maintained the post-flash of 12 electrons, as exposure times for the three primary standard images are only a few seconds, and the resulting sky background is low. It is in this regime that the post-flash is effective at mitigating CTE losses. Moving the target to the readout corner and applying corrections that model the CTE losses completely mitigate CTE-related systematic effects. Left uncorrected, these could cause an underestimate of the flux of our targets.

Moving the primary target to the readout corner in Cycle 22 had the additional advantage of allowing us to select just the center of the UVIS2-C512C-SUB subarray, rather than the UVIS1-FIX array used in Cycle 20. ${ }^{22}$ This significantly reduced the instrument readout and data transfer overhead at the cost of fewer sources on the frame with which to check relative photometry. We judged this trade-off to be acceptable, given the small number of secondary sources on the frame in passbands bluer than F625W. Additionally, in Cycle 22 we moved the target from the IR-UVIS-FIX aperture to the IR-FIX aperture, as the former is affected by a row of bad pixels. While our reduction pipeline accounted for this row in the analysis of the Cycle 20 data, we prefer to avoid systematic issues prior to

\footnotetext{
22 The pixel coordinates and sizes of the WFC3 apertures are defined in http://www.stsci.edu/hst/observatory/apertures/wfc3.html.
} 
image acquisition, rather than relying on data reduction procedures to mitigate their impact.

\section{6. $A B$ as the Spectrophotometric Reference}

In N16, we reported magnitudes for our DA WD stars relative to Vega. Bohlin (2014, hereafter B14) sets the normalization of the three primary standards of SED relative to the Vega flux at $5556 \AA$ (in air, or $5557.5 \AA$ in vacuum), $F^{\text {Vega }}(5556 \AA)=3.44 \times 10^{-9} \mathrm{erg} \mathrm{cm}^{-2} \AA^{-1} \ldots{ }^{-1}$ measured from HST/STIS spectrophotometry. This monochromatic flux is a reconciliation of the Megessier (1995) flux in the visible with the Midcourse Space Experiment mid-IR fluxes. It is the single tie point from the CALSPEC primary standards to Vega, and the only location where consistency between the flux of Vega and the flux of the CALSPEC stars is guaranteed. The statistical uncertainty on this monochromatic measurement, and thus the absolute calibration of the primary standards, is $0.5 \%$. Any errors in this absolute calibration propagate to all wavelengths and so do not affect the shapes of the SEDs.

There are, however, considerable systematic uncertainties in the HST/STIS-based SED model of Vega. ${ }^{23}$ Some of these uncertainties arise from the flux calibration of the HST/STIS spectra, which exhibit considerable saturation. B14 estimates an additional $0.2 \%-0.5 \%$ systematic uncertainty with wavelength in the visible. There are likely additional systematic errors in the calibration of Vega in the infrared, due to the presence of a dust disk. B14 reports a $\sim 1 \%$ discrepancy in the IR flux of Vega from IRAC measurements, despite including a three-component model for the dust disk (with its own uncertainties) that has not been resolved to date. ${ }^{24}$ Establishing high-precision spectrophotometric standards, only to report their measurements with respect to a spectrophotometric reference that itself suffers from known systematic errors, is contrary to the goals of our work.

Ideally, we would report an absolute flux in units such as janskys; however, our experiment only establishes SEDs relative to the three CALSPEC primary standards-our work cannot improve on the B14 $0.5 \%$ statistical uncertainty on the absolute calibration of the primary standards. Moreover, absolute fluxes in janskys are not as widely used as magnitudes for optical measurements, and they are not accepted as inputs or reported as outputs by many image processing pipelines (e.g., photpipe; Rest et al. 2005) and are therefore inconvenient for many purposes. We do not report Vega-based magnitudes in this work despite their widespread use in the WD literature, and instead report $\mathrm{AB}$ magnitudes with zeropoints determined from the three CALSPEC standards. This choice implicitly assumes that the primary standards are on the same AB flux scale (Oke \& Gunn 1983). Fukugita et al. (1996) define the relationship between $\mathrm{AB}$ magnitudes and physical fluxes such that a source with constant spectral flux density per unit frequency $F_{\nu}=3631$ Jy has magnitude 0 in all passbands. The $\mathrm{AB}$ source and Vega are not merely interchangeable spectrophotometric references that define different photometric systems. The AB source is purely a construct, whereas Vega is a time-variable source on the sky with a complex SED that

\footnotetext{
23 Specifically the B14 model for the SED of Vega included in CALSPEC: alpha_lyr_stis_008.fits.

${ }^{24}$ The best current estimate of the systematic uncertainty of the CALSPEC flux system is determined from the three DA WD primary standards and is reported in the covariance matrix included with the CALSPEC data products: WDcovar_001.fits.
}

suffers from wavelength-dependent systematic errors that are likely irreducible. Fundamentally, the DA WDs are better spectrophotometric references than Vega itself.

It is not possible to validate our assumption that the CALSPEC flux scale is an absolute scale within the framework developed in Section 6. Several ground-based photometric catalogs also assume that the CALSPEC flux scale is an accurate absolute flux scale, and we can only measure a relative offset in each passband. Quantifying any error in the absolute calibration of the CALSPEC SEDs requires independent data, and we discuss a framework for establishing such absolute flux standards in Section 9 using a combination of the DA WD atmospheres themselves and laboratory standards. With this caveat, $\mathrm{AB}$ magnitudes have the great benefit of being directly related to physical units and remaining compatible with widely used photometry routines used to determine the zero-points of optical and infrared images, while avoiding any of the systematic issues that arise with Vega as a spectrophotometric reference.

\subsection{Additional Spectroscopy for Testing the Consistency of Our Analysis}

As with our Cycle 20 targets, we obtained spectra for our Cycle 22 targets using the Gemini Multi Object Spectrograph (GMOS; Hook et al. 2004) on both the Gemini-North and Gemini-South telescopes. While we reduced our slit width from 1".5 to $1^{\prime \prime}$ for Gemini/GMOS spectra obtained for Cycle 22 targets, as the excellent seeing on Maunakea warranted the narrower slit, we made no other major changes to our GMOS observing program. In addition to the GMOS spectra, we obtained a second spectrum for a subset of our targets using the Blue Channel spectrograph on the MMT in Cycle 20. We found these additional spectra to be invaluable, as they provided a cross-check on the inference of the intrinsic parameters of the DA WDs presented in N16. While the MMT/Blue Channel spectra typically have lower resolution than the Gemini/GMOS spectra (2-3 $\AA$ pixel ${ }^{-1}$ vs. $0.92 \AA$ pixel $^{-1}$ ) and lower $\mathrm{S} / \mathrm{N}$ on average, as we had less allocated observing time, they cover a longer range in wavelength. This allows us to model the $\mathrm{H} \alpha$ feature that is truncated in our Gemini/GMOS spectra.

The MMT/Blue Channel spectra can be flux-calibrated more accurately than the Gemini/GMOS spectra, due to a combination of factors: (i) On MMT/Blue Channel, the trace is dispersed across a single CCD readout by a single amplifier, whereas the trace is dispersed across three CCDs, each read out by four amplifiers on Gemini/GMOS, requiring careful gain matching and ad hoc flux adjustment corrections. (ii) The Gemini/GMOS observations were executed in queue mode, and a standard spectrum was not guaranteed to be observed on the same nights as our targets. With MMT/Blue Channel, we always obtained multiple observations of at least one, and typically two to three, blue spectrophotometric standards, spaced across the night for calibration of our DA WDs. (iii) We typically obtained a quartz flat and $\mathrm{HeNeAr}$ lamp spectrum at each pointing with MMT/Blue Channel. Finally, we obtained several spectra of the primary standard GD 71 with the Goodman spectrograph on the Southern Astrophysical Research telescope (SOAR) to flux-calibrate spectra of our Cycle 25 targets. These high-S/N spectra are useful, as they not only test the internal consistency of the analysis but also test the consistency of the SED of GD 71, inferred 
independently from each spectrum, with the CALSPEC SED of GD 71.

\section{Deriving Cross-cycle Photometric Catalogs}

In this section, we develop a model to derive the apparent $\mathrm{AB}$ magnitudes of our program stars from their instrumental measurements. Combining HST photometric measurements from any multicycle program requires care, as the detector sensitivity evolves nonmonotonically with time. In N16, we asserted that as our measurements were from a single Cycle 20 program and the evolution of the sensitivity over the cycle was likely to be small, we could rely on the zero-points measured by the ongoing HST Servicing Mission Observatory Verification (SMOV) calibration program and reported on MAST. N16 unearthed systematic differences between the sensitivity of UVIS1 and UVIS2 that were unmodeled at the time; even if the zero-points were precisely measured, they were not accurate for the UVIS2 chip. These sensitivity differences have now been accounted for by chip-dependent calibrations in the calwf 3 pipeline and MAST data products (Deustua et al. 2016, 2017).

Nevertheless, the fiducial zero-points provided by Space Telescope staff are time averaged and are not suitable for experiments that require high-precision photometric calibration of multicycle imaging such as ours. This shortcoming motivated us to add intra-Cycle 22 monitoring of the three primary standards to our program, allowing us to tie our targets directly to the CALSPEC system without any intermediate transformations. In order to combine our data from Cycles 20 and 22, we must account for the stochastic and systematic effects in $H S T$ /WFC3 while modeling the primary standard observations together with our DA WD observations. We begin by examining these effects from independent archival $H S T$ data sets and then develop a model that accounts for these effects in order to derive the calibrated photometry of our DA WDs.

\subsection{Accounting for HST/WFC3 Sensitivity Evolution}

Tracking the change in sensitivity of an instrument over time requires the continuous monitoring of an external reference with the same configuration. GRW $+70^{\circ} 5824$ (hereafter GRW70) was originally established as a standard by Oke (1990) (itself concerned with establishing a faint network of spectrophotometric stars- $5.5 \mathrm{mag}$ brighter in $V$ on the mean than the objects in this work). Space Telescope staff have been monitoring the WD standard GRW70 in several HST/WFC3 UVIS passbands (Shanahan et al. 2017) since Cycle 17. Using archival calwf3 processed images, we measured the flux of GRW70 using a 10-pixel radius from exposures where the star was located on the same subarray as our targets. The dithers between different observations are small, so we do not expect errors in flat-fielding with position. Figure 4 shows the measured magnitudes of GRW70 over time, assuming constant sensitivity.

The sensitivity function inferred from the GRW70 observations in all the passbands is complex, exhibiting a steep increase right after launch, when WFC3 was added to the HST instrument payload, likely due to outgassing. Over time, the sensitivity decreases, but there are sharp changes within each cycle that do not correlate strongly with any physical parameters of the spacecraft. We model the long-term sensitivity decrease with a simple linear trend. The standard deviation of the residuals about the linear trend is $5-8 \mathrm{mmag}$ in the observed passbands, $2-3$ times

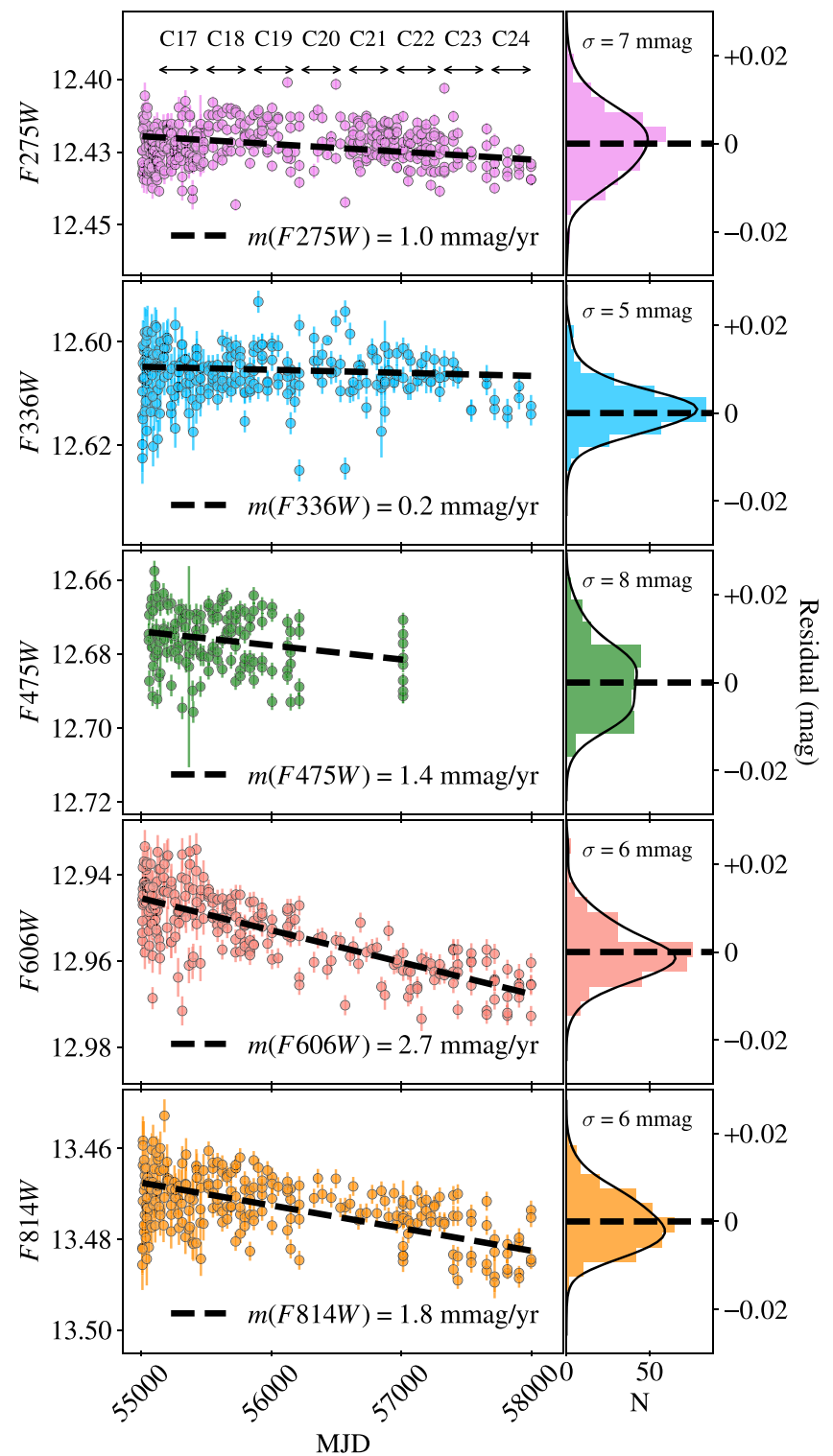

Figure 4. Sensitivity evolution of $H S T$ WFC3 UVIS2 determined by STScI staff from repeated measurements of the standard GRW70, assuming a constant zero-point. The star has been monitored starting with Cycle 17, when WFC3 was added to the HST payload as part of the SMOV program. HST cycle numbers corresponding to MJD ranges are indicated in the top panel. The mean trend in each passband can be characterized as a linear decline of typically

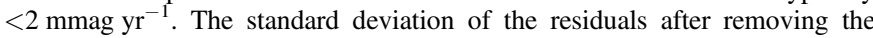
linear trend is $5-8$ mmag. This dispersion is larger than the photometric uncertainties and is readily apparent among even closely spaced observations. We note that the GRW70 photometry is determined from images reduced by the calwf3 pipeline, and it is likely that some of the dispersion is a result of the suboptimal image processing. In addition, there are sources of dispersion intrinsic to $H S T / \mathrm{WFC} 3$, such as spacecraft breathing (optics), charge persistence (detector), instabilities in the amplifiers (electronics), and flatfielding errors as a function of time (image processing). This figure illustrates the extent and timescale of secular response variations. While we do not use the GRW70 data directly, we account for this dispersion in the model for our observations in Section 4.2. We note that the standard error of the mean can be much smaller than the dispersion of the individual measurements.

larger than the photometric uncertainties. A single measurement may have a low error but may still differ significantly from a subsequent measurement, as the repeatability of the HST/WFC3 detector is not captured by the photon noise. This excess scatter 
motivates the introduction of an intrinsic dispersion in Section 4.2 to explain our $H S T / \mathrm{WFC} 3$ observations.

We model the additional 2-4 mmag of dispersion as intrinsic to $H S T$ /WFC3. This is reasonable, as this additional dispersion is a result of a combination of spacecraft breathing, persistence, instability in the amplifier electronics, and flat-fielding errors as a function of time. We cannot disentangle these effects with currently available data. While the linear decrease in the

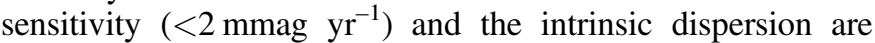
small, both effects must be modeled in order to combine our Cycle 20 and Cycle 22 measurements. Unfortunately, measurements of GRW70 are not available in all of our program's passbands. Given the limitations of this data set, we do not use the GRW70 observations to set zero-points when deriving apparent magnitudes from our multicycle observations, but we do include an intrinsic dispersion to account for measurement repeatability.

\subsection{Modeling Cycle 20 and 22 Photometry and Tying Measurements to CALSPEC}

We construct a hierarchical model to describe our instrumental measurements from Cycles 20 and 22 in each passband. The model parameters are (i) the zero-point in each passband; (ii) an intrinsic dispersion in each passband to model the scatter in the measurements that is in excess of the estimate from the photon noise; (iii) a single offset between the Cycle 20 and Cycle 22 observations in each passband; (iv) the number of degrees of freedom of a Student's $t$-distribution, used to model the small fraction of instrumental measurements, that are afflicted by cosmic rays and are outliers; and (v) the desired apparent magnitudes of each source. We discuss each of these parameters below:

1. Determining the zero-point in each passband $Z$ requires stars with measured instrumental magnitudes and known apparent magnitudes. We determine the zero-points using our observations of three CALSPEC primary standards and their synthetic magnitudes derived from the CALSPEC SEDs. ${ }^{25}$ As the CALSPEC primary standards are also DA WDs with very similar colors to our program stars, and as they were observed contemporaneously with the same instrumental configuration, we can apply the zero-point derived from the three primary standards directly to our program stars.

2. As discussed in Section 4.1, the observed scatter of the multicycle GRW70 measurements is underestimated by the photometric errors. This additional scatter likely arises from multiple effects, and it is not possible to disentangle each of them. The residuals between the GRW70 observations and a linear trend to account for zero-point evolution over time are normally distributed. This motivates the introduction of a single parameter to model the excess dispersion in each passband $\sigma_{\text {int. }}$. While this intrinsic dispersion magnitude can be constrained from instrumental measurements of all the stars, we must also account for any systematic difference in the instrumental response between HST cycles.

3. In optimizing our observing program between Cycle 20 and Cycle 22 (see Section 3.5), we changed the on-

25 The specific CALSPEC SED models used are gd71_mod_010.fits for GD 71, gd153_mod_010.fits for GD 153, and gd191b2b_mod_010.fits for G191-B2B. detector location of our targets from UVIS1-FIX to UVIS2-C512-C-SUB, and from IR-UVIS-FIX to IR-FIX. Consequently, we must account for the difference in zeropoint caused by the change in the system response arising from the change in location, and from any evolution in the overall throughput between Cycles 20 and 22. We introduce a single parameter in each passband $\Delta Z_{\mathrm{C} 20}$ to model this offset. This offset can only be constrained using stars that were observed in both Cycles 20 and 22 . In particular, it is not necessary to model the instrumental observations in $\mathrm{F} 275 \mathrm{~W}$, as all these measurements were obtained in Cycle 22.

4. We measure the brightness of our sources from the individual PAM- and CTE-loss-corrected flc images, but we do not apply any cosmic-ray rejection, as we found that this affects the shape of the PSF (see Section 3.4). Without cosmic-ray rejection, some fraction of the measurements will be impacted by cosmic rays within the aperture, and assuming that the measurements are normally distributed would bias the estimate of the zeropoints. Our model must therefore account for nonnormally distributed outliers. C19 uses sigma-clipping of each star's measurements and a bi-weight estimator to determine mean instrumental magnitudes for the three primary standards. Hogg et al. (2010) caution against the sigma-clipping procedure, as it does not optimize an objective function, and the results are dependent on the initial guess.

We express the probability density of the data given the model parameters as a Student's $t$-distribution, as an alternative to the normal distribution (see Chap. 17.2 of Gelman et al. 2004). In the limit of the number of degrees of freedom $\nu$ growing to infinity, the Student's $t$ distribution is equivalent to the normal distribution, but for smaller values, the $t$-distribution has heavier tails, allowing robust inference in the presence of outliers. As the fraction of outliers is small, and there are nearly 200 observations across all stars in each passband, we leave the number of degrees of freedom as a free hyperparameter of the model.

5. Finally, in addition to these four hyperparameters of our hierarchical model in each passband, we introduce a parameter for the apparent magnitude of each of our program DA WD stars. These are the latent variables of the model that are needed to calibrate the SED of each program star in Section 6.

The probability density of our observations of the $p$ th CALSPEC primary standard given the model parameters is the likelihood function

$$
\begin{aligned}
P\left(\widehat{\boldsymbol{m}}_{\boldsymbol{p}} \mid m_{p}, Z, \sigma_{\mathrm{int}}^{2}, \nu\right)= & \prod_{i=1}^{N_{p}} T\left(\widehat{m}_{p, i} \mid m_{p}-Z,\left(\sigma_{\mathrm{int}}^{2}\right.\right. \\
& \left.\left.+\widehat{\sigma}_{p, i}^{2}\right)^{-1}, \nu\right),
\end{aligned}
$$

where $\widehat{m}_{p, i}$ is the $i$ th observed instrumental magnitude with photometric measurement error described by an estimated variance $\widehat{\sigma}_{p, i}^{2}$ for CALSPEC standard $p$ from the set of $N_{p}=$ 3 primary standards. We discuss the derivation of the synthetic magnitudes in each passband $m_{p}$ from the SEDs of the CALSPEC primary standards in the following section (Section 4.3). $T(y \mid \mu, \lambda, \nu)$ generically denotes a Student's 
$t$-distribution in $y$ centered on $\mu$ with inverse scale parameter $\lambda$ and $\nu$ degrees of freedom. As we began monitoring the primary standards in Cycle 22, their observations only set the zero-point $Z$ and do not constrain the zero-point offset between Cycles 20 and $22 \Delta Z_{\mathrm{C} 20}$. The synthetic magnitudes of the CALSPEC primary standards are determined for an infinite aperture, and the zero-points implicitly incorporate the aperture correction from a radius of 7.5 pixels in the UVIS bands and a radius of 5 pixels in the F160W.

In the limit of $\nu$ growing to infinity, the Student's $t$-distribution reduces to a normal distribution. Conditional on knowing the value of $\sigma_{\mathrm{int}}$, the zero-point $Z$ can be estimated from the conventional weighted mean difference between the apparent magnitudes and the mean instrumental magnitudes of the three CALSPEC primary standards:

$$
\hat{Z}=\left(\sum_{p=1}^{3} \sum_{i=1}^{N_{p}} w_{p, i}\right)^{-1} \sum_{p=1}^{3} \sum_{i=1}^{N_{p}} w_{p, i} \cdot\left(m_{p}-\widehat{m}_{p, i}\right),
$$

where the weights are the inverse variances: $w_{p, i}^{-1}=\sigma_{\text {int }}^{2}+\widehat{\sigma}_{p, i}^{2}$. Within our model, the measurements of the secondary standards inform us of both the values of $\sigma_{\text {int }}$ and finite $\nu$, which in turn influence our posterior estimate of $Z$ and its uncertainty. Hence, our approach jointly models the primary and secondary standards in a single coherent Bayesian model, with the marginal posterior estimate of $Z$ obtained by computationally marginalizing the full posterior, Equation (14), over all other parameters using MCMC. This process fundamentally ties the HST photometry of our DA WDs to a flux scale defined by the weighted mean of the three CALSPEC primary standards. This panchromatic HST photometry is used to normalize our inferred SED models in Section 6.

For the secondary standards, we model the probability density of our observations given the parameters with the likelihood function

$$
\begin{aligned}
& P\left(\widehat{\boldsymbol{m}}_{s} \mid m_{s}, Z, \Delta Z_{\mathrm{C} 20}, \sigma_{\mathrm{int}}^{2}, \nu\right)=\prod_{i=1}^{n_{s}^{\mathrm{C} 22}} T\left(\widehat{m}_{s, i} \mid m_{s}-Z,\left(\sigma_{\mathrm{int}}^{2}+\widehat{\sigma}_{s, i}^{2}\right)^{-1}, \nu\right) \\
& \quad \times \prod_{j=1}^{n_{s}^{\mathrm{C2} 20}} T\left(\widehat{m}_{s, j} \mid m_{s}-Z-\Delta Z_{\mathrm{C} 20},\left(\sigma_{\mathrm{int}}^{2}+\widehat{\sigma}_{s, j}^{2}\right)^{-1}, \nu\right),
\end{aligned}
$$

where $\widehat{m}_{s, i}$ is the $i$ th observed instrumental magnitude with photometric measurement error described by an estimated variance $\widehat{\sigma}_{s, i}^{2}$ for a DA WD star $s$ from the set of $N_{S}$ standards $\left\{s: s=1, \ldots, N_{S}\right\}$ with $n_{s}^{\mathrm{C} 20}$ observations in Cycle 20 and $n_{s}^{\mathrm{C} 22}$ observations in Cycle 22. The latent (true) magnitude of this star $m_{s}$ is related to the instrumental magnitudes in Cycle 22 through the additive zero-point $Z$, and the Cycle 22 zero-point is related to the Cycle 20 zero-point through an additive offset $\Delta Z_{\mathrm{C} 20}$. The intrinsic dispersion $\sigma_{\text {int }}$ accounts for the failure of the photometric uncertainties $\widehat{\sigma}_{s, i}$ to capture the full variance of the data $\widehat{m}_{s, i}$.

We depict the model in Figure 5 as a directed acyclic graph, a probabilistic graphical representation of our hierarchical Bayesian model. The probabilistic graphical model illustrates how the unknown apparent magnitudes $m_{s}$ of each of our program DA WD stars (labeled by an index $s$ ) are related to the

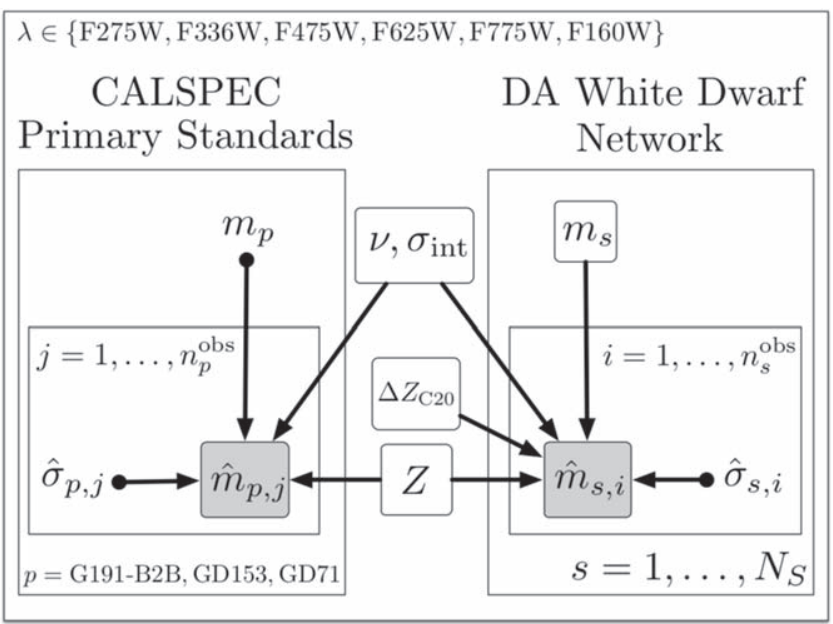

Figure 5. Directed acyclic graph depicting the hierarchical model for our instrumental photometry. Clear rounded rectangles denote model parameters, while shaded rounded rectangles denote measurements. Arrows that originate at circles denote inputs that are combined with the measurements. Flat rectangular "plates" with subscripts indicate the product of likelihoods over the indicated variables $\{i, j, p, s, \lambda\}$. The instrumental magnitudes $\widehat{m}$ and their uncertainties $\widehat{\sigma}$ for the CALSPEC primary standards $p$ and the DA WDs $s$ are modeled by a Student's $t$-distribution with $\nu$ degrees of freedom about the difference between each star's apparent magnitudes $m$ and the zero-point $Z$. The synthetic apparent magnitudes of the CALSPEC primary standards $m_{p}$ set the zero-point, which is transferred to determine the apparent magnitudes of the DA WD network stars $m_{s}$. The intrinsic dispersion $\sigma_{\text {int }}$ accounts for the photometric measurements underestimating the variance of the measurements, while the zero-point offset $\Delta Z_{\mathrm{C} 20}$ accounts for the difference in the zero-point between Cycle 20 and Cycle 22 from changing the detector location and time evolution of the sensitivity.

measurements of the CALSPEC primary standards (labeled by an index $p$ ) and the model hyperparameters in each passband: the zero-point $Z$, the offset between Cycle 20 and Cycle 22 $\Delta Z_{\mathrm{C} 20}$, the intrinsic dispersion $\sigma_{\mathrm{int}}$, and the number of degrees of the Student's $t$-distribution used to model photometric outliers.

\subsection{Synthetic Photometry}

Evaluating the likelihood of the hierarchical model described in the previous section requires the magnitudes of the three CALSPEC primary standards in our program passbands. These are derived using synthetic photometry of the CALSPEC SEDs (see footnote 25) through the model of the transmission for each passband (see footnote 21 ). We briefly summarize the synthetic photometry procedure here. The same procedure is used in Section 6 when determining the SED of each DA WD using the observed spectroscopy and the apparent magnitudes derived in this section.

The synthetic flux of a source with spectral flux density $F^{p}(\lambda)$ through the photon response function of each of the $H S T$ /WFC3 passbands $R(\lambda)$ is defined as

$$
\left\langle F_{\lambda}^{p}\right\rangle=\frac{\int_{0}^{\infty} \lambda \cdot F^{p}(\lambda) \cdot R(\lambda) \cdot d \lambda}{\int_{0}^{\infty} \lambda \cdot R(\lambda) \cdot d \lambda} .
$$

The apparent magnitudes of the primary standards $m_{p}$ are inputs derived from the ratio of the synthetic flux of their CALSPEC 
SEDs to the synthetic flux of the spectrophotometric reference:

$$
m_{p}=-2.5 \log \left(\frac{\int_{0}^{\infty} \lambda \cdot F^{p}(\lambda) \cdot R(\lambda) \cdot d \lambda}{\int_{0}^{\infty} \lambda \cdot F^{\mathrm{AB}}(\lambda) \cdot R(\lambda) \cdot d \lambda}\right),
$$

where $F_{\lambda}^{\mathrm{AB}}$ is the spectral flux density of the fiducial $\mathrm{AB}$ source expressed in erg cm ${ }^{-2} \mathrm{~s}^{-1} \AA^{-1}$. Throughout the text and the data products provided together with this work, synthetic magnitudes are computed using Equation (5), consistent with our convention of reporting the observed spectral flux density per unit wavelength $F_{\lambda} . F_{\lambda}^{\mathrm{AB}}$ is related to $F_{\nu}^{\mathrm{AB}}=3631 \mathrm{Jy}$ by

$$
F_{\lambda}^{\mathrm{AB}}=F_{\nu}^{\mathrm{AB}} \cdot \frac{\nu^{2}}{c}
$$

for $\lambda=c / \nu$.

With Equation (6), it is also possible to define the synthetic flux following Koornneef et al. (1986) as

$$
\left\langle F_{\nu}^{p}\right\rangle=\frac{\int_{0}^{\infty} \nu^{-1} \cdot F^{p}(\nu) \cdot R(\nu) \cdot d \nu}{\int_{0}^{\infty} \nu^{-1} \cdot R(\nu) \cdot d \nu}
$$

and the synthetic magnitude as

$$
\begin{aligned}
m_{p} & =-2.5 \log \left(\frac{\int_{0}^{\infty} \nu^{-1} \cdot F^{p}(\nu) \cdot R(\nu) \cdot d \nu}{\int_{0}^{\infty} \nu^{-1} \cdot F^{\mathrm{AB}}(\nu) \cdot R(\nu) \cdot d \nu}\right) \\
& =-2.5 \log \left(\frac{\int_{0}^{\infty} \nu^{-1} \cdot F^{p}(\nu) \cdot R(\nu) \cdot d \nu}{F^{\mathrm{AB}} \cdot \int_{0}^{\infty} \nu^{-1} \cdot R(\nu) \cdot d \nu}\right) \\
& =-2.5 \log \left(\frac{\left\langle F_{\nu}^{p}\right\rangle}{1 \mathrm{erg} \cdot \mathrm{cm}^{-2} \cdot \mathrm{s}^{-1} \cdot \mathrm{Hz}^{-1}}\right)-48.60 \mathrm{mag},
\end{aligned}
$$

where the last simplification to the form provided by Oke \& Gunn (1983) is possible, as the spectral flux density of the $\mathrm{AB}$ standard is constant per unit frequency, i.e., $F_{\nu}^{\mathrm{AB}} \equiv$ $3.631 \times 10^{-20} \mathrm{erg} \mathrm{cm}^{-2} \mathrm{~s}^{-1} \mathrm{~Hz}^{-1}$. If the synthetic flux is expressed in janskys, the appropriate zero-point is instead $2.5 \log \left(\frac{3631 \mathrm{Jy}}{1 \mathrm{Jy}}\right)=8.90 \mathrm{mag}$.

Equation (6) holds for all monochromatic values of the flux density and is also valid for the total flux through a passband, i.e., the integral of the flux density in Equation (5) when computed at the "pivot" wavelength $\lambda_{\text {pivot }}$ (Koornneef et al. 1986):

$$
\lambda_{\text {pivot }}=\sqrt{\frac{\int_{0}^{\infty} \lambda \cdot R(\lambda) \cdot d \lambda}{\int_{0}^{\infty} R(\lambda) / \lambda \cdot d \lambda}} .
$$

The pivot wavelength is a characteristic of the passband and can be used to convert our AB-based magnitudes to the synthetic flux derived from a source reported with spectral flux density per unit wavelength $\left\langle F_{\lambda}\right\rangle$ without knowledge of the underlying source SED. The pivot wavelength differs from the effective wavelength $\lambda_{\text {eff }}$,

$$
\lambda_{\mathrm{eff}}=\frac{\int_{0}^{\infty} \lambda^{2} \cdot F^{p}(\lambda) \cdot R(\lambda) \cdot d \lambda}{\int_{0}^{\infty} \lambda \cdot F^{p}(\lambda) \cdot R(\lambda) \cdot d \lambda},
$$

which is source dependent and is the mean wavelength of photons detected through a passband. The effective wavelength is necessary when comparing apparent magnitudes of a source observed through a passband directly with the calibrated model SED of the source.

\subsection{The Probability Density of the HST Observations}

The full probability density of the data $\mathcal{D} \equiv\left\{\widehat{\boldsymbol{m}}_{\boldsymbol{p}}, \widehat{\boldsymbol{m}}_{\boldsymbol{s}}\right\}$ given the model parameters $\boldsymbol{\Phi} \equiv\left\{\left\{\boldsymbol{m}_{\boldsymbol{s}}\right\}, Z, \Delta Z_{\mathrm{C} 20}, \sigma_{\text {int }}^{2}, \nu\right\}$ and the CALSPEC magnitudes $\left\{m_{p}\right\}$ is the product of the likelihoods for the DA WD network stars (Equation (1)) and the primary standards (Equation (3)):

$$
\begin{aligned}
P\left(\mathcal{D} \mid \boldsymbol{\Phi},\left\{m_{p}\right\}\right)= & \prod_{p=1}^{N_{p}} P\left(\widehat{\boldsymbol{m}}_{\boldsymbol{p}} \mid m_{p}, Z, \sigma_{\mathrm{int}}^{2}, \nu\right) \\
& \times \prod_{s=1}^{N_{S}} P\left(\widehat{\boldsymbol{m}}_{\boldsymbol{s}} \mid m_{s}, Z, \Delta Z_{\mathrm{C} 20}, \sigma_{\text {int }}^{2}, \nu\right) .
\end{aligned}
$$

\subsection{Priors}

We model the prior as separable functions on each of the model parameters. We follow Gelman (2006) and use weakly informative priors on all the parameters of our hierarchical model. We use a uniform prior on the apparent magnitudes $m$ for $8 \mathrm{mag} \leqslant m \leqslant 25 \mathrm{mag}$. This weakly informative prior spans a large enough range to encompass all of the observed magnitudes of the primary and program standards in all of our observed passbands. We use a normal distribution, denoted by $N(\mu, \sigma)$ with standard deviation $\sigma=1 \mathrm{mag}$ and mean $\mu$ equal to the average difference of the input apparent magnitude and the measured instrumental magnitudes for the three primary standards $\left\langle m_{p}-\widehat{m}_{p, j}\right\rangle_{p, j}$. This prior is only weakly informative, as the true zero-points have uncertainties on the order of a few millimagnitudes, rather than the width of 1 mag we have used in our prior. We use an $N(0,1 \mathrm{mag})$ distribution as the prior on the zero-point offset between Cycles 20 and $22 \Delta Z_{C 20}$, which is weakly informative, as our analysis in Section 4.1 constrains the change to be at most a few millimagnitudes with similar uncertainties. We use a half-Cauchy distribution with $\beta=1 \mathrm{mag}$ on the intrinsic dispersion $\sigma_{\text {int }}$, as this quantity must always be positive and is known to be at most a few millimagnitudes from our analysis in Section 4.1. Finally, we employ a half-Cauchy distribution with $\beta=5$ on the number of degrees of freedom of the Student's $t$-distribution $\nu$, as this parameter must always be positive, and we expect the fraction of outliers to be small. Assuming prior independence of all the parameters, the full joint prior distribution is the product of the 
marginal priors on each of the parameters:

$$
\begin{aligned}
m_{s} & \sim U(8 \mathrm{mag}, 25 \mathrm{mag}) \\
Z & \sim N\left(\mu=\left\langle m_{p}-\widehat{m}_{p, j}\right\rangle_{p, j}, \sigma=1 \mathrm{mag}\right) \\
\Delta Z_{\mathrm{C} 20} & \sim N(\mu=0 \mathrm{mag}, \sigma=1 \mathrm{mag}) \\
\sigma_{\text {int }} & \sim H C\left(x_{0}=0 \mathrm{mag}, \beta=1 \mathrm{mag}\right) \\
\nu & \sim H C\left(x_{0}=0, \beta=5\right) \\
P(\boldsymbol{\Phi}) & =P(Z) \cdot P\left(\Delta Z_{\mathrm{C} 20}\right) \cdot P\left(\sigma_{\text {int }}^{2}\right) \cdot P(\nu) \cdot \prod_{s=1}^{N_{S}} P\left(m_{s}\right) .
\end{aligned}
$$

As there are alternative parameterizations in the literature, we define the probability density of a half-Cauchy random variable $x \sim H C\left(x_{0}, \beta\right)$ as

$$
P\left(x \mid x_{0}, \beta\right)=\frac{2}{\pi} \cdot\left[\beta \cdot\left(1+\frac{\left(x-x_{0}\right)^{2}}{\beta^{2}}\right)\right]^{-1}
$$

for $x \geqslant 0$ and zero otherwise.

The hierarchical model is conditioned on the apparent magnitudes $m_{p}$ of the primary standards, which are used to infer the zero-point $Z$ given the instrumental magnitudes of the primary standards. This zero-point is then coherently propagated to our network of stars within the hierarchical model, thus incorporating the covariance of the zero-point and the apparent magnitudes of our DA WDs.

\subsection{Posterior Distribution and Estimation}

The full posterior distribution of the model $\boldsymbol{\Phi}$ given the data $\mathcal{D}$ is proportional to the product of the likelihood (Equation (11)) and the prior (Equation (12))

$$
\begin{aligned}
P\left(\boldsymbol{\Phi} \mid \mathcal{D},\left\{m_{p}\right\}\right) \propto P\left(\mathcal{D} \mid \boldsymbol{\Phi},\left\{m_{p}\right\}\right) \cdot P(\boldsymbol{\Phi}) \\
=\prod_{p=1}^{3} P\left(\widehat{\boldsymbol{m}}_{p} \mid m_{p}, Z, \sigma_{\mathrm{int}}, \nu\right) \\
\quad \times\left[\prod_{s=1}^{N_{S}} P\left(\widehat{\boldsymbol{m}}_{\boldsymbol{s}} \mid m_{s}, Z, \Delta Z_{\mathrm{C} 20}, \sigma_{\mathrm{int}}, \nu\right) \cdot P\left(m_{s}\right)\right] \\
\quad \times P(Z) \cdot P\left(\Delta Z_{\mathrm{C} 20}\right) \cdot P\left(\sigma_{\mathrm{int}}\right) \cdot P(\nu) .
\end{aligned}
$$

The model is solved for the $N_{S}+4$ parameters in each of the program passbands independently. As we did not obtain F275W observations in Cycle 20, there is no zero-point offset defined for this passband. C19 report measurements using three separate photometry packages: DAOPHOT (Stetson 1987), SourceExtractor, and ILAPH, a custom interactive aperture photometry developed by one of us (A.S.). C19 find that the DAOPHOT and ILAPH measurements show reasonable agreement, while both differ systematically from the SourceExtractor measurements. They determine this to be a result of how SourceExtractor treats the sky background. DAOPHOT is also known to exhibit systematic differences (Bajaj \& Khandrika 2017) to careful aperture photometry performed with the PhotUtils Python package (Bradley et al. 2017) or the APER routine available through the IDL Astronomy Library. ${ }^{26}$ We analyze the ILAPH measurements in this work, as these IDL procedures were developed specifically for measuring count rates from our flc images.

\footnotetext{
${ }^{26}$ https://idlastro.gsfc.nasa.gov/
}

We use the No U-Turn Sampler (NUTS; Homan \& Gelman 2014) implemented in the pymc 3 package (Salvatier et al. 2016) to estimate the posterior distribution. We run four independent Markov chains initialized to different positions for 20,000 steps, following a burn-in of 2000 steps that are discarded. We use a suite of diagnostic tests, including visually inspecting the chains for convergence, verifying that the chain autocorrelation lengths are small, determining that the Gelman-Rubin statistic (Equation (20) from Gelman \& Rubin 1992) is near unity, and comparison of our inferred zero-points against the fiducial values determined from the SMOV program and reported on MAST. Additionally, we check that the outliers to the Student's $t$-distribution are all brighter than the mean apparent magnitudes of our DA WDs $m_{s}$, consistent with cosmic rays. Only 2-8 out of $\sim 190$ distinct observations in each passband are flagged as outliers. The intrinsic dispersion $\sigma_{\text {int }}$ is between 1 and 4 mmag, decreasing from F275W to F775W. The apparent magnitudes of our DA WD stars are listed in Table 1.

\section{Selection Cuts}

Up to this point, the analysis has been independent of any assumptions about the physical nature of the sources being modeled-we do not need to specify temperature, surface gravity, and extinction to determine the mean apparent magnitudes with respect to the three CALSPEC primary standards. However, in order to infer accurate SEDs from our $H S T /$ WFC3 photometry and spectroscopy of our DA WDs, we must specify a model for the observations. This model fundamentally assumes that our objects are isolated, photometrically stable sources that are well described by NLTE purehydrogen atmospheres that do not exhibit strong magnetic fields (see Section 2.3). Not all of our targets satisfy this condition-these must be excluded from further analysis.

Kleinman et al. (2013) identify SDSS J041053 as a DA+M: E binary system. Eisenstein et al. (2006), Kleinman et al. (2013), and several other sources identify SDSS J172135 as a DA WD with $T_{\text {eff }} \sim 9450 \mathrm{~K}$, well below our cutoff of $20,000 \mathrm{~K}$ to exclude pulsating WDs, and even more distant from our initial $T_{\text {eff }}$ estimate of $30,000 \mathrm{~K}$. We attempted to determine how SDSS J172135 was included in our list of Cycle 22 targets, despite being more than $10,000 \mathrm{~K}$ cooler than our lower limit in $T_{\text {eff. }}$. We determined that the object was initially shortlisted as a possible target in 2012, prior to our Cycle 20 program. At that time, either the spectrum of a different object was inspected, due to a failure in name resolution-it is unlikely to be a coincidence that SDSS J172132 is a DA WD with a $T_{\text {eff }} \sim 30,000 \mathrm{~K}$ listed directly above SDSS J172135 in the Montreal White Dwarf Database ${ }^{27}$ —or the temperature was somehow grossly overestimated. Unfortunately, as the object was shortlisted for our Cycle 20 program, it was not scrutinized again prior to Cycle 22, and we failed to flag it as an unsuitable target. We find that the SED parameters of both SDSS J041053 and SDSS J172135 inferred using the methodology in Section 6 place them near the edge of our DA WD atmosphere grid with significant extinction, and their photometric residuals are two orders of magnitude larger than the rest of our sample. We cannot exclude SDSS J041053 and SDSS J172135 on the basis of our existing temporal observations, but given the binarity of the former and the subthreshold temperature of the latter, we feel that excluding them from our network is justified.

\footnotetext{
${ }^{27}$ http://www.montrealwhitedwarfdatabase.org/
} 
Table 1

Apparent AB Magnitudes and Photometric Uncertainties of the Network of DA White Dwarfs and CALSPEC Primary Standards

\begin{tabular}{|c|c|c|c|c|c|c|c|c|}
\hline Object & $\begin{array}{c}\text { R.A. } \\
\text { (hh:mm:ss) }\end{array}$ & $\begin{array}{c}\delta \\
\left(\circ: !^{\prime \prime}\right)\end{array}$ & F275W & F336W & $\begin{array}{r}\mathrm{F} 475 \mathrm{~W} \\
\mathrm{AB}\end{array}$ & mag) & F775W & F160W \\
\hline G191-B2B & 05:05:30.61 & $+52: 49: 51.96$ & $10.4904(1)$ & $10.8902(1)$ & $11.4988(1)$ & 12.0307 (1) & $12.4514(1)$ & $13.8853(2)$ \\
\hline GD 153 & $12: 57: 02.34$ & $+22: 01: 52.68$ & $12.2016(2)$ & $12.5679(1)$ & $13.0998(2)$ & $13.5976(1)$ & $14.0017(1)$ & $15.4139(2)$ \\
\hline GD 71 & $05: 52: 27.61$ & $+15: 53: 13.75$ & $11.9888(1)$ & $12.3360(1)$ & $12.7988(1)$ & $13.2790(1)$ & $13.6720(1)$ & $15.0676(2)$ \\
\hline SDSS J010322 & 01:03:22.19 & $-00: 20: 47.73$ & 18.1952 (4) & $18.5268(5)$ & $19.0833(5)$ & $19.5686(5)$ & $19.9648(6)$ & $21.3552(12)$ \\
\hline SDSS J022817 & $02: 28: 17.17$ & $-08: 27: 16.41$ & $19.5183(8)$ & $19.7152(10)$ & $19.8151(7)$ & $20.1690(7)$ & $20.5014(6)$ & $21.7371(17)$ \\
\hline SDSS J024854 & $02: 48: 54.96$ & $+33: 45: 48.30$ & $17.8285(4)$ & $18.0400(6)$ & $18.3696(3)$ & $18.7459(3)$ & $19.0773(2)$ & $20.3400(6)$ \\
\hline SDSS J041053 ${ }^{\mathrm{a}}$ & $04: 10: 53.63$ & $+06: 30: 27.75$ & $18.1162(9)$ & $18.4041(4)$ & $18.8796(5)$ & $19.2537(3)$ & $19.3936(5)$ & $19.4982(5)$ \\
\hline WD $0554^{\mathrm{a}}$ & 05:57:01.30 & $-16: 35: 12.00$ & $16.7760(5)$ & $17.1531(3)$ & $17.7271(5)$ & $18.2205(3)$ & $18.6172(5)$ & $20.0431(7)$ \\
\hline SDSS J072752 & $07: 27: 52.76$ & $+32: 14: 16.10$ & $17.1636(3)$ & $17.4715(3)$ & $17.9933(3)$ & $18.4567(2)$ & $18.8370(3)$ & $20.2166(7)$ \\
\hline SDSS J081508 & 08:15:08.78 & $+07: 31: 45.80$ & $18.9505(6)$ & $19.2635(8)$ & $19.7162(5)$ & $20.1838(5)$ & 20.5794 (6) & $21.9616(24)$ \\
\hline SDSS J102430 & $10: 24: 30.93$ & $-00: 32: 07.03$ & $18.2606(18)$ & $18.5143(4)$ & $18.9042(5)$ & $19.3174(4)$ & $19.6649(10)$ & 20.9905 (13) \\
\hline SDSS J111059 & $11: 10: 59.43$ & $-17: 09: 54.10$ & $17.0406(3)$ & $17.3544(4)$ & $17.8668(3)$ & $18.3135(2)$ & $18.6887(2)$ & $20.0566(5)$ \\
\hline SDSS J111127 & 11:11:27.30 & $+39: 56: 28.00$ & $17.4429(4)$ & $17.8298(6)$ & $18.4206(3)$ & $18.9390(4)$ & $19.3441(3)$ & 20.7975 (9) \\
\hline SDSS J120650 & 12:06:50.41 & $+02: 01: 42.46$ & $18.2397(4)$ & $18.4888(4)$ & 18.6719 (4) & $19.0601(3)$ & $19.4112(7)$ & 20.7027 (9) \\
\hline SDSS J121405 & 12:14:05.11 & $+45: 38: 18.50$ & $16.9401(2)$ & $17.2827(2)$ & $17.7606(2)$ & $18.2362(3)$ & $18.6292(2)$ & $20.0378(4)$ \\
\hline SDSS J130234 & 13:02:34.44 & $+10: 12: 39.01$ & $16.1879(2)$ & $16.5216(2)$ & $17.0364(2)$ & $17.5140(2)$ & $17.9037(2)$ & $19.3031(4)$ \\
\hline SDSS J131445 & $13: 14: 45.05$ & $-03: 14: 15.64$ & $18.2577(4)$ & $18.5969(5)$ & $19.1018(5)$ & $19.5668(5)$ & $19.9553(9)$ & $21.3284(12)$ \\
\hline SDSS J151421 & $15: 14: 21.27$ & $+00: 47: 52.79$ & $15.1100(2)$ & $15.3907(2)$ & $15.7090(2)$ & $16.1202(2)$ & $16.4712(1)$ & $17.7870(4)$ \\
\hline SDSS J155745 & $15: 57: 45.40$ & $+55: 46: 09.70$ & 16.4999 (2) & $16.8766(2)$ & $17.4702(3)$ & 17.9917 (2) & $18.3880(2)$ & $19.8343(5)$ \\
\hline SDSS J163800 & $16: 38: 00.36$ & $+00: 47: 17.81$ & $18.0158(8)$ & 18.3177 (4) & 18.8399 (5) & $19.2808(3)$ & $19.6605(5)$ & 20.9963 (9) \\
\hline SDSS J172135 & $17: 21: 35.98$ & $+29: 40: 16.00$ & $20.3714(13)$ & $20.0782(16)$ & $19.6559(5)$ & $19.6699(3)$ & $19.7678(3)$ & $20.5520(21)$ \\
\hline SDSS J181424 & $18: 14: 24.13$ & $+78: 54: 02.90$ & 15.7913 (2) & $16.1213(2)$ & $16.5441(2)$ & $17.0056(2)$ & $17.3926(1)$ & $18.7857(2)$ \\
\hline SDSS J203722 ${ }^{\mathrm{a}}$ & $20: 37: 22.17$ & $-05: 13: 03.03$ & $18.2568(7)$ & $18.5438(4)$ & $18.9428(6)$ & $19.3504(12)$ & $19.6718(10)$ & $20.9790(23)$ \\
\hline SDSS J210150 & 21:01:50.66 & $-05: 45: 50.97$ & $18.0681(4)$ & $18.3344(4)$ & $18.6560(3)$ & $19.0636(2)$ & 19.4140 (4) & $20.7396(8)$ \\
\hline SDSS J232941 & $23: 29: 41.33$ & $+00: 11: 07.80$ & 17.9434 (4) & $18.1090(4)$ & $18.1607(6)$ & $18.4697(3)$ & $18.7753(7)$ & 19.9949 (6) \\
\hline SDSS J235144 & $23: 51: 44.29$ & $+37: 55: 42.60$ & $17.4494(4)$ & $17.6619(3)$ & $18.0751(3)$ & $18.4595(3)$ & $18.7868(2)$ & $20.0747(4)$ \\
\hline \multirow[t]{2}{*}{ Parameter } & & & F275W & F336W & F475W & F625W & F775W & F160W \\
\hline & & & \multicolumn{6}{|c|}{$\mathrm{AB}$ mag (mmag) } \\
\hline Zero-point $Z$ & & & $24.0596(1)$ & 24.5899 (1) & $25.5774(1)$ & $25.4056(1)$ & 24.7189 (1) & $25.8116(1)$ \\
\hline Offset $\Delta Z_{\mathrm{C} 20}$ & & & $\cdots(\cdots)$ & $-0.0326(3)$ & $-0.0091(4)$ & $-0.0139(2)$ & $+0.0089(4)$ & $-0.0125(5)$ \\
\hline Dispersion $\sigma_{\text {int }}$ & & & $0.0031(1)$ & $0.0017(1)$ & $0.0026(1)$ & $0.0015(1)$ & $0.0006(1)$ & 0.0045 \\
\hline$\nu$ (dimensionless) & & & $2.451(0.435)$ & $1.626(0.212)$ & $2.275(0.406)$ & $3.186(0.714)$ & $1.380(0.151)$ & $2.978(0.542)$ \\
\hline
\end{tabular}

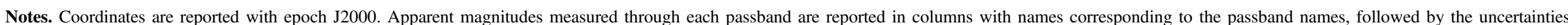

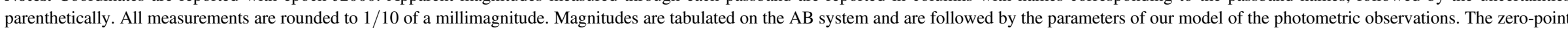

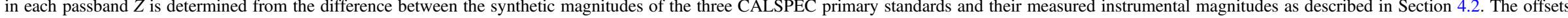

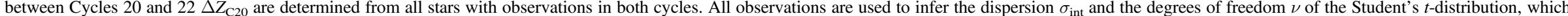

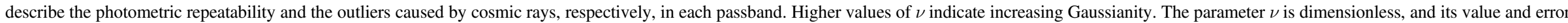
are reported to three decimal places.

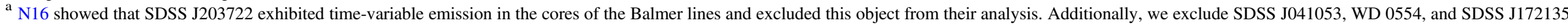
in this work-see Section 5 for details. Their measured apparent magnitudes are listed here for completeness. 
C19 report that WD 0554 is photometrically unstable with a 0.2 mag peak-to-peak amplitude and a Welch-Stetson variability index (Welch \& Stetson 1993) I of 3.98. The inferred $\log g$ parameter of WD 0554 is $\sim 9$ dex, near the edge of the grid and more than 1 dex higher than the mean of our sample. To verify the high surface gravity inferred from our MMT/ Blue Channel spectrum, we obtained a second high-S/N and high-resolution spectrum with the Inamori Magellan Areal Camera and Spectrograph (IMACS) on Magellan-Baade. Our inferred surface gravity remains consistent irrespective of which spectrum is used in the analysis. This anomalously high surface gravity and photometric variability suggest that WD 0554 has a weak magnetic field with unresolved Zeeman splitting leading to line broadening and an overestimate of the surface gravity. Finally, N16 found that SDSS J203722 exhibited time-variable emission in the cores of the Balmer lines. C19 also report that this object has several time-resolved observations from the LCO network and is photometrically unstable with $I=3.35$ and a standard deviation of observations $\sigma=0.04 \mathrm{mag}$, well above the mean standard deviation of field stars, which have $\sigma \sim 0.01 \mathrm{mag}$. We therefore exclude SDSS J041053, WD 0554, SDSS J172135, and SDSS J203722 from additional analysis.

\section{Forward-modeling the DA WD Spectroscopy and Photometry}

In order to establish our DA WDs as spectrophotometric standards on an equal footing with the three CALSPEC primary standards, we must determine robust SEDs, spanning the wavelength range from the UV to the NIR (roughly $1350 \AA-2.5 \mu \mathrm{m})$, for each of our stars. The most direct approach to inferring the intrinsic and extrinsic parameters and deriving an SED is to forward-model the observations of each DA WD - the apparent magnitudes presented in Table 1 and the spectroscopy presented in C19. In this section, we describe the N16 analysis and address its shortcomings with an improved methodology developed for this work.

\subsection{Comparison to N16 Methodology}

N16 developed a fitter around the Tlusty synthetic DA WD atmosphere grid to infer the intrinsic parameters $T_{\text {eff }}$ and $\log g$ from spectra. Using a standard methodology first introduced in Bergeron et al. (1992a, hereafter B92), a local linear continuum is fit across each of the hydrogen Balmer lines, and the line profile is extracted and normalized to have a constant continuum equal to unity. The same features are extracted from the model atmosphere and used to fit the spectral lines, with the log-likelihood defined as the sum of the average sum of squared differences between data and model for $\mathrm{H} \beta$ through $\mathrm{H} \zeta$. The best-fit intrinsic parameters are used to construct an unreddened SED model. N16 attributed the difference between the unreddened synthetic magnitudes and the HST observations to reddening, which, once inferred, was used to correct the unreddened SED model. The key feature of this methodology is that it divides the inferences of the model parameters into two discrete steps: inference of the intrinsic DA WD parameters, followed by inferences of the extrinsic reddening parameters.
More detailed analysis of our growing data set revealed two shortcomings of the N16 analysis when applied to lower-S/N spectroscopic data from our faint DA WDs:

1. Lack of propagation of uncertainty from the local linear continuum fit to the intrinsic parameters: Despite the heteroskedasticity of measured error in the spectrum flux, the N16 analysis does not incorporate any notion of uncertainty, largely because the spectroscopic reductions were preliminary. While it is possible to incorporate the measurement errors by modifying the log-likelihood to be the sum of the chi-square statistic of each of the Balmer lines, this is inadequate, as the flux calibration errors are also correlated with wavelength and are not independent or identically distributed. The N16 analysis attempted to fit a local pseudo-continuum to each of the Balmer lines and determine the intrinsic DA WD parameters purely from the shape of these features. The local continuum between the Balmer lines becomes less pronounced or is completely absent for $\mathrm{H} \gamma$ and bluer, and this local linear model exhibits increasing bias with decreasing temperature and increasing surface gravity. The parameters of the local linear continuum model are treated as "nuisance" variables and seldom reported, and it is not possible to coherently propagate any uncertainties in these parameters into the SED flux. We also find that the method underestimates the uncertainties on the inferred parameters by as much as a factor of 4-10 for the four stars presented in N16.

2. Not simultaneously modeling the intrinsic DA WD parameters and the extrinsic reddening: Our DA WDs are impacted by reddening, which affects the shape of the entire spectrum, including the wide Balmer lines. The B92 method infers the intrinsic WD parameters solely from the Balmer lines. The reddening was determined serially, asserting that the difference between the synthetic photometry of the unreddened SED and the observations was solely due to reddening. This procedure neglected the reddening of the spectra and therefore may lead to a biased estimate of the intrinsic WD parameters, particularly for our distant DA WDs, where we expect the effect of extinction to be larger than for nearby stars. N16 attempted to mitigate this by using an estimate of the reddening derived from SDSS photometry to deredden the spectrum prior to estimating the intrinsic DA WD parameters. The N16 framework is iterative-an estimate of the reddening is needed to estimate the unreddened SED, which is in turn used to derive the reddening, and the procedure can be repeated until a predefined convergence criterion is satisfied. While N16 used independent data to derive the initial and final reddening estimate, the methodology could not fully account for the covariance between the intrinsic DA WD parameters and extrinsic reddening, as it followed the B92 approach of splitting the inference of these two quantities into different steps.

We developed the WDmodel methodology to address these shortcomings and coherently forward-model all of the observations. We detail the various components of the WDmodel and define the likelihood of the observations given the model in the following sections. 


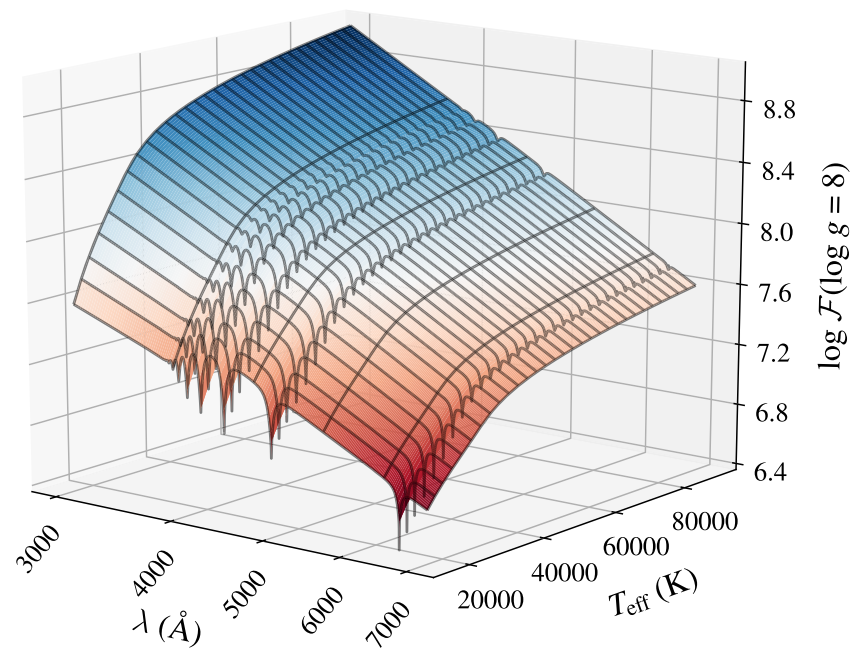

Figure 6. Slice with $\log g=8$ through the Tlusty v202 DA WD atmosphere model grid used in this work. We interpolate the grid with logarithmic scaling of wavelength and flux $\mathcal{F}$ to reduce errors. The model clearly exhibits the increasingly blue continuum and decreasing equivalent width of the Balmer lines with the increase in $T_{\text {eff }}$ exhibited by real observations in Figure 2.

\subsection{The DA WD Atmosphere Grid and Intrinsic Parameters}

We describe the unreddened DA WD SED with two parameters, $T_{\text {eff }}$ and $\log g$. We use the same Tlusty (Hubeny \& Lanz 1995) v202 NLTE model atmosphere grid $^{28}$ as N16. The grid has 31 uneven steps in $T_{\text {eff }}$ from 16,000 to $90,000 \mathrm{~K}$, with a spacing of $2000 \mathrm{~K}$ from 16,000 to $20,000 \mathrm{~K}$ and $2500 \mathrm{~K}$ from 20,000 to $90,000 \mathrm{~K}$. The grid has six even steps in $\log g$ from 7 to 9.5 dex, with 0.5 dex spacing. The ogrid covers a wavelength range of $1350 \AA-2.7 \mu \mathrm{m}$, in $1 \AA$ steps from $1350 \AA \leqslant \lambda \leqslant 3000 \AA, \quad 0.5 \AA$ steps from $3000 \AA \leqslant \lambda \leqslant$ $7000 \AA$, and $5 \AA$ steps for $\lambda>7000 \AA$. A slice through the grid is shown in Figure 6. The grid uses all available hydrogen line profiles from Tremblay \& Bergeron (2009). The treatment of level dissolution and pseudocontinua follows Hubeny et al. (1994).

While there are DA WD stars with intrinsic parameters outside the range covered by our grid, we do not use them as spectrophotometric standards at this time. In particular, we limit our consideration to DA WDs with $T_{\text {eff }}>16,000 \mathrm{~K}$, as there are still model uncertainties about the treatment of convection and the mixing-length prescription for cooler DA WDs (Bergeron et al. 1992b). Some of these sources may exhibit variability as described in Section 2.3, and such sources are not suitable for use as spectrophotometric standards. This selection cut is conservative but is justified, as our primary goal is to establish an all-sky network of standards with a minimal set of theoretical assumptions. We emphasize that our WDmodel code should not be used to model DA WDs cooler than $16,000 \mathrm{~K}$ and will report erroneously high values of $A_{V}$, as the only way to redden the model below the lower limit of the grid is to add extinction. A new model atmosphere grid that extends to lower temperatures could in principle be used together with our WDmodel code to fit cool $\left(T_{\text {eff }}<16,000 \mathrm{~K}\right)$ DA WDs. When modeling spectroscopy together with photometry to establish spectrophotometric standards, though, we make the fundamental assumption that the WD is not variable and the model is stationary. This assumption does

\footnotetext{
${ }^{28}$ http://nova.astro.umd.edu/Tlusty2002/tlusty-frames-refs.html
}

not hold unless the spectroscopy and photometry are obtained contemporaneously.

The logarithm of SED model flux is tri-linearly interpolated at any $T_{\text {eff }}, \log g$, and $\log (\lambda)$ within the bounds of the grid and then exponentiated to determine the SED model $\mathcal{F}_{s}\left(T_{\text {eff }}, \log g, \lambda\right)$ for a DA WD $s$. Levenhagen et al. (2017) have made a higher-resolution grid of atmosphere models available, but this new grid exhibits a discontinuity where it transitions from LTE atmospheres for $T_{\text {eff }}<34,000 \mathrm{~K}$ to NLTE atmospheres for $T_{\text {eff }} \geqslant 34,000 \mathrm{~K}$, whereas our Tlusty grid is NLTE throughout.

\subsection{The Extrinsic Reddening Parameters}

We adopt the F99 model to describe the wavelength dependence of the extinction due to interstellar dust. F99 is defined for $1150 \AA \leqslant \lambda \leqslant 6 \mu \mathrm{m}$ and uses the Fitzpatrick \& Massa (1990) model of interstellar extinction for $\lambda \leqslant 2700 \AA$ and a spline model above. The extinction at any wavelength is specified by two parameters, $A_{V}$ and $R_{V}$. We apply extinction $A_{s}\left(A_{V}, R_{V}, \lambda\right)$ to the unreddened SED model $\mathcal{F}_{s}\left(T_{\text {eff }}, \log g, \lambda\right)$ to determine the reddened SED model $\widetilde{F_{s}}$ for a DA WD $s$ :

$$
\begin{aligned}
& \widetilde{F}_{S}\left(T_{\text {eff }}, \log g, A_{V}, R_{V}, \lambda\right) \\
& \quad=\mathcal{F}_{s}\left(T_{\text {eff }}, \log g, \lambda\right) \cdot 10^{-0.4 \cdot A_{s}\left(A_{V}, R_{V}, \lambda\right)} .
\end{aligned}
$$

The WDmodel code supports any reddening law implemented in the extinction (Barbary 2016) Python module. As in N16, we do not find any significant difference in our results when using the O'Donnell (1994) reddening law.

\subsection{The Normalization of Synthetic Photometry}

We compute synthetic magnitudes from the reddened SED $\widetilde{F}_{S}$ using Equation (5). We add a single achromatic normalization parameter $\mu$ to the synthetic reddened magnitudes in all passbands to account for the distance and radius of the DA WD and thereby model the apparent magnitudes inferred from the observations in Section 4. Normalizing the flux of the reddened SED $\widetilde{F}_{s}$ with $\mu$ results in the final calibrated SED $\boldsymbol{F}$ tied to the CALSPEC system:

$$
\begin{aligned}
& \boldsymbol{F}_{s}\left(T_{\mathrm{eff}}, \log g, A_{V}, R_{V}, \mu, \lambda\right) \\
& \quad=\widetilde{F_{s}}\left(T_{\text {eff }}, \log g, A_{V}, R_{V}, \lambda\right) \cdot 10^{-0.4 \cdot \mu} .
\end{aligned}
$$

While $\mu$ is not the distance modulus, as the absolute magnitude of each WD is not known without imposing a massradius-luminosity relation, it is functionally equivalent. The difference between the HST/WFC3 photometry and the unreddened synthetic magnitudes for three of our DA WDs are illustrated together with the inferred extinction curves in Figure 7.

\subsection{The Probability Density of the HST Apparent Magnitudes}

The probability density of the set of photometric data $\left\{m_{s}\right\}$ reported in Table 1 given the model parameters $\left\{T_{\mathrm{eff}}, \log g, A_{V}, R_{V}, \mu\right\}$ is the likelihood function

$$
\begin{aligned}
P & \left(\left\{m_{s}\right\} \mid T_{\mathrm{eff}}, \log g, A_{V}, R_{V}, \mu\right) \\
& =\prod_{\lambda=1}^{N_{\mathrm{PB}}} N\left(m_{s, \lambda} \mid M_{s, \lambda}\left(T_{\mathrm{eff}}, \log g, A_{V}, R_{V}\right)+\mu, \sigma_{s, \lambda}\right),
\end{aligned}
$$

where $m_{s, \lambda}$ is the apparent magnitude for a DA WD star $s$ with photometric measurement error described by an estimated 


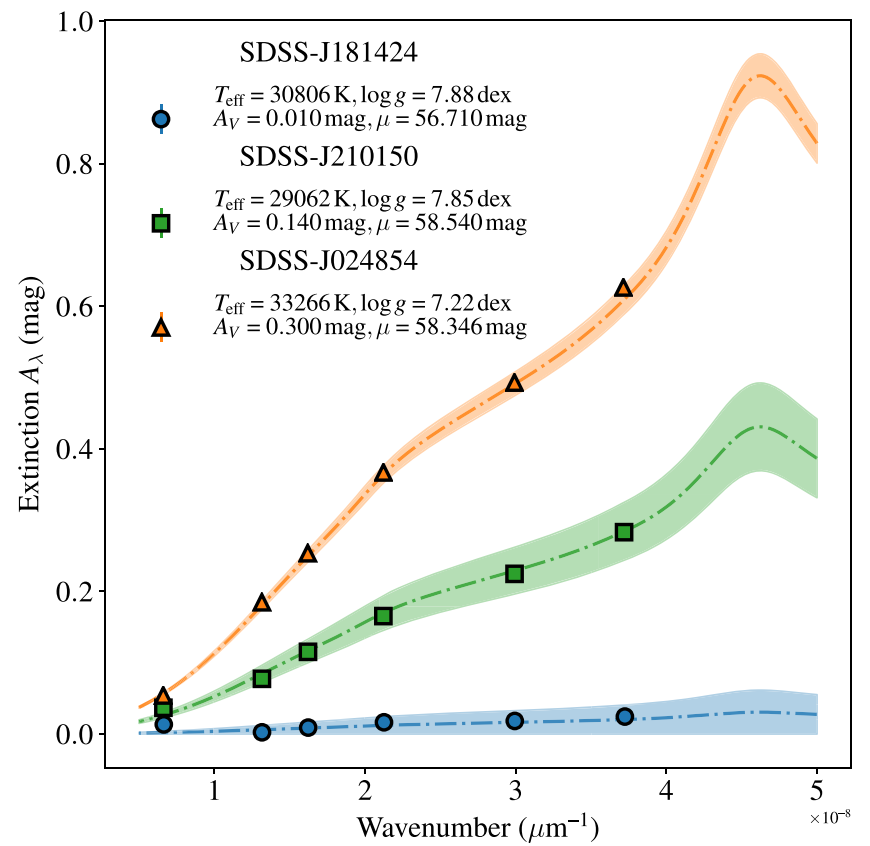

Figure 7. F99 extinction curves (dot-dashed lines) for our inferred values of $A_{V}$ with $R_{V}=3.1$ for three of our DA WDs. The markers show the difference between the observed HST/WFC3 photometry and the synthetic magnitudes of the unreddened model in each passband at the effective wavelength of the reddened SED. Model parameters are indicated in the legend. The shaded region encompasses the $1 \sigma$ uncertainty on the observations and the model parameters. Despite the stars having comparable $T_{\text {eff }}$, there is a wide range in the line-of-sight extinction that must be accounted for when modeling the observations of each star.

standard deviation $\sigma_{s \lambda}$, and $M_{s, \lambda}\left(T_{\text {eff }}, \log g, A_{V}, R_{V}\right)$ is the synthetic magnitude of the reddened SED $\widetilde{F}_{S}$ through $H S T /$ WFC3 passband $\lambda \in\{$ F275W, F336W, F475W, F625W, F775W, F160W\}.

\subsection{Accounting for Spectral Resolution and Overall Normalization}

Modeling the observed spectra $\mathcal{S}_{s}$ of DA WD $s$ is more complex. Minimally, (i) a normalization parameter applied to the reddened SED to match the observed flux and (ii) the resolution of the reddened, normalized SED model must be degraded to the resolution of the observed spectrum to account for the seeing at the observatory and the configuration of the spectrograph. We normalize the extinguished model SED for a DA WD $s$ using a single parameter $d_{L}$ :

$$
\begin{gathered}
F_{s}\left(T_{\mathrm{eff}}, \log g, A_{V}, R_{V}, d_{L}, \lambda\right) \\
=\frac{\widetilde{F_{S}}\left(T_{\mathrm{eff}}, \log g, A_{V}, R_{V}, \lambda\right)}{4 \pi \cdot d_{L}^{2}},
\end{gathered}
$$

where $F_{s}$ is the extinguished, normalized SED constructed from the reddening SED model $\widetilde{F}_{s}$. As with $\mu$, this parameter is not the true luminosity distance to each WD, as the absolute flux of each WD is not known without imposing a mass-radiusluminosity relation.

We then convolve $F_{s}$ with a Gaussian kernel to model the observed spectral resolution, as illustrated in Figure 8. The standard deviation $\sigma_{R}$ of the kernel is related to the FWHM of

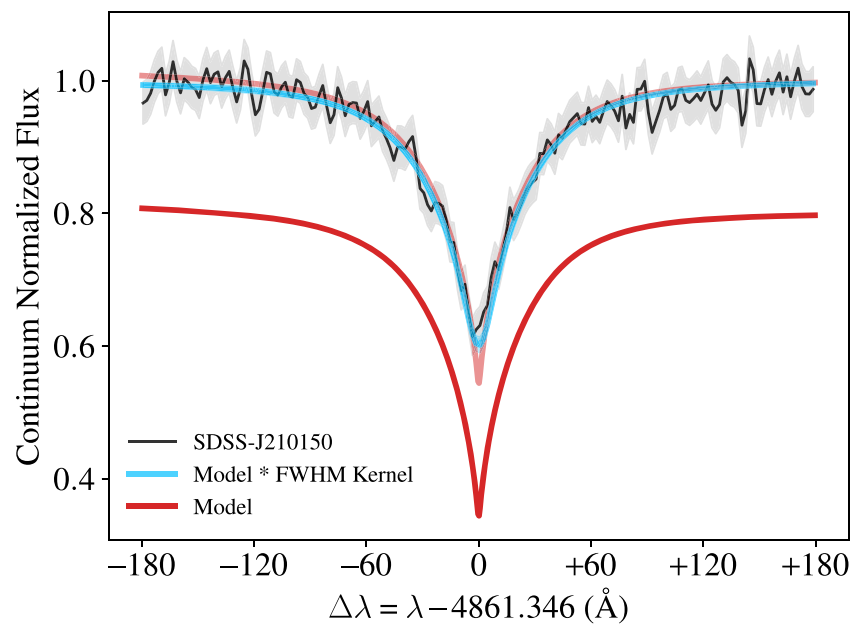

Figure 8. Model $F$ (in red) has a higher spectral resolution than the data $\mathcal{S}$ (in black), and the effect is particularly evident in the cores of the Balmer features (light red, offset to match the data), such as $\mathrm{H} \beta$ for one of our DA WDs. This can lead to biases in the inferred intrinsic DA WD parameters, as $T_{\text {eff }}$ and $\log g$ are sensitive to the shapes of the Balmer lines. We convolve the model with a Gaussian kernel with standard deviation proportional to the FWHM (in blue) to match the observed spectrum.

the spectrum by

$$
\sigma_{R} \equiv \frac{\text { FWHM }}{R \cdot \sqrt{8 \cdot \ln (2)}},
$$

where $R$ is the median resolution of the observed spectrum in $\AA$ per spectral unit, while the remaining factor in the denominator is the ratio of the FWHM to the standard deviation of a normal distribution. The convolution kernel is truncated at $\pm 4 \sigma_{R}$. The normalized model of the observed spectrum is

$$
\begin{aligned}
& f_{s}\left(T_{\text {eff }}, \log g, A_{V}, R_{V}, d_{L}, \text { FWHM, } \lambda\right) \\
& \quad=F_{s}\left(T_{\text {eff }}, \log g, A_{V}, R_{V}, d_{L}, \lambda\right) * N\left(1, \sigma_{R}(\text { FWHM })\right) .
\end{aligned}
$$

\subsection{Accounting for Correlated Errors in the Flux Calibration of the Spectrum}

If the shape of spectra were free of error, these two parameters would suffice; however, as we note in Section 2.2, flux calibration of ground-based spectroscopy is fraught with potential sources of systematic error. The SEDs of the standard stars that are used to flux-calibrate the spectra of our DA WDs are often less well determined than the DA WDs themselves. In addition, the flux corrections applied to the blue $(\leqslant 4200 \AA)$ and red ends $(\geqslant 7000 \AA)$ of the spectra are large, as the throughput drops sharply. The difficulty of determining an unbiased flux correction in the red is compounded by the intrinsic faintness of the DA WDs at these wavelengths and the fringing in the detector. The flux correction is typically modeled with loworder splines or piecewise polynomials. These functions are multiplied by the instrumental flux of the spectrum to produce the calibrated flux. Errors in the flux correction can exhibit ringing arising from polynomials and splines overfitting the data. The flux correction is typically only accurate to a few percent, and any error in the calibration procedure results in a nonmonotonic error in the shape of the spectrum.

The residual vector describes the correlated error between the flux of the observed spectrum $\mathcal{S}_{s}$ and the flux of the model 
spectrum $f_{s}$ :

$$
r\left(\lambda_{i}\right)=\mathcal{S}_{s}\left(\lambda_{i}\right)-f_{s}\left(T_{\mathrm{eff}}, \log g, A_{V}, R_{V}, d_{L}, \mathrm{FWHM}, \lambda_{i}\right) .
$$

We model this correlated error with a Gaussian process (GP; see Rasmussen \& Williams 2005 and references therein for a detailed background). We construct the covariance kernel of the Gaussian process as the sum of a Matérn 3/2 kernel to describe the correlated error and a white-noise kernel to describe the dispersion in the observed flux of the spectrum that is underestimated by the reported uncertainty on the flux. This kernel can be expressed as

$$
\begin{aligned}
& k\left(f_{\sigma}, \tau, f_{\omega}, \Delta \lambda_{i j}\right) \\
& =\left(f_{\sigma} \cdot \bar{\sigma}_{\mathcal{S}}\right)^{2} \cdot\left[1+\frac{\sqrt{3} \cdot \Delta \lambda_{i j}}{\tau}\right] \cdot \exp \left(-\frac{\sqrt{3} \cdot \Delta \lambda}{\tau}\right) \\
& \quad+\left(f_{\omega} \cdot \bar{\sigma}_{\mathcal{S}}\right)^{2} \cdot \delta_{i j},
\end{aligned}
$$

where $\Delta \lambda_{i j} \equiv\left|\lambda_{i}-\lambda_{j}\right|$ is the absolute value of the difference between any two wavelengths $\lambda_{i}$ and $\lambda_{j}, \tau$ is the characteristic length scale of correlations in the spectrum, $f_{\sigma}$ and $f_{\omega}$ are the scale-free amplitudes of the correlated error and white-noise components, respectively, and $\delta_{i j}$ is the Kronecker delta. The scale-free amplitudes are scaled by the median of the reported uncertainty in the spectroscopic flux $\bar{\sigma}_{\mathcal{S}}$ to model an observed spectrum of a DA WD $\mathcal{S}_{s}$.

The Matérn $3 / 2$ kernel is the simplest singly differentiable functional in the Matérn covariance family. These functionals can be represented as the product of a polynomial and exponential for all half-integer values of the kernel order. In the limit of order of the kernel tending to infinity, the functional reduces to a squared exponential kernel. As the squared exponential is infinitely differentiable, the Gaussian process becomes sensitive to structure on any length scale, including sharp noise spikes. The low-order Matérn 3/2 kernel can describe the large-scale errors in the flux calibration of the spectrum and incurs the lowest computational cost, while remaining insensitive to sharp features that arise because of the finite $\mathrm{S} / \mathrm{N}$ of our observations. The model error is a realization of a zero mean Gaussian process $\mathcal{G P}$ with the kernel

$$
G(\lambda) \sim \mathcal{G P}\left[0, k\left(f_{\sigma}, \tau, f_{\omega}, \Delta \lambda_{i j}\right)\right] .
$$

For a spectrum of length $N_{\lambda}$, the full $N_{\lambda} \times N_{\lambda}$ covariance matrix $\boldsymbol{C}$ incorporates both the reported errors in the observed flux of the spectrum, $\sigma_{\mathcal{S}}$, and the model error parameterized by the Gaussian process. The covariance matrix can be expressed as

$$
C_{i j}=\sigma_{\mathcal{S}}^{i} \cdot \delta_{i j}+k\left(f_{\sigma}, \tau, f_{\omega}, \Delta \lambda_{i j}\right) .
$$

We model the corrected spectrum $S$ as the sum of the normalized model spectrum $f_{s}$ and the spectral flux calibration error, represented by a realization of a GP with the above kernel:

$$
\begin{aligned}
& S_{s}\left(T_{\text {eff }}, \log g, A_{V}, R_{V}, d_{L}, \text { FWHM, } \lambda\right) \\
& \quad=f_{s}\left(T_{\text {eff }}, \log g, A_{V}, R_{V}, d_{L}, \text { FWHM, } \lambda\right)+G(\lambda) .
\end{aligned}
$$

We infer the parameters of the latent GP jointly with the other model parameters. We marginalize over the latent GP to propagate this uncertainty into the parameter inference. We illustrate the effectiveness of the Gaussian process at modeling the correlated error in the flux calibration in Figure 9. We find that the Gaussian process corrects for the errors in the flux calibration for DA WD spectra observed with a variety of different instruments, including MMT/Blue Channel, GeminiN/GMOS, and SOAR/Goodman. Unlike other ad hoc methods that we attempted to use, including smoothing splines and various families of polynomials, the Gaussian process does not require fine-tuning and can incorporate the reported uncertainties on the flux of the spectrum.

Czekala et al. (2015) also use a Gaussian process to model the correlated offsets between observed and model spectra. Their Starfish ${ }^{29}$ package is designed to work with highresolution echelle spectra that are not flux-calibrated, and the Gaussian process is used to capture the additional variance in observed spectral features that is not captured by stellar atmosphere template libraries. While we cannot employ the Starfish package directly for this work, its approach to inferring a stellar atmosphere to model an observed spectrum is conceptually similar to ours.

\subsection{The Probability Density of the Ground-based Spectrum}

The natural logarithm of the probability density of the ground-based spectrum $\mathcal{S}_{s}$ for a DA WD $s$ given the model parameters $\left\{T_{\text {eff }}, \log g, A_{V}, R_{V}, d_{L}, \mathrm{FWHM}, f_{\sigma}, \tau, f_{\omega}\right\}$ is the log-likelihood function

$$
\begin{gathered}
\ln P\left(\mathcal{S}_{s} \mid T_{\mathrm{eff}}, \log g, A_{V}, R_{V}, d_{L}, \text { FWHM, } f_{\sigma}, \tau, f_{\omega}\right) \\
=-\frac{1}{2}\left[\boldsymbol{r}^{\mathrm{T}} \cdot \boldsymbol{C}^{-1} \cdot \boldsymbol{r}+\ln |2 \pi \boldsymbol{C}|\right],
\end{gathered}
$$

where $\boldsymbol{r}$ is the residual vector.

\subsection{The Full Probability Density of Observations}

The full probability density of the observations of a DA WD $s, \mathcal{D}_{s} \equiv\left\{\left\{m_{s}\right\}, \mathcal{S}_{s}\right\}$, given the model parameters $\boldsymbol{\Phi} \equiv$ $\left\{T_{\text {eff }}, \log g, A_{V}, R_{V}, d_{L}, \mu\right.$, FWHM, $\left.f_{\sigma}, \tau, f_{\omega}\right\}$ is the product of the likelihoods for the HST photometry (Equation (17)) and the ground-based spectroscopy (Equation (26)):

$$
\begin{aligned}
P\left(\mathcal{D}_{s} \mid \boldsymbol{\Phi}\right) & \\
= & P\left(\left\{m_{s}\right\} \mid T_{\mathrm{eff}}, \log g, A_{V}, R_{V}, \mu\right) \\
\quad & \times P\left(\mathcal{S}_{s} \mid T_{\mathrm{eff}}, \log g, A_{V}, R_{V}, d_{L}, \text { FWHM, } f_{\sigma}, \tau, f_{\omega}\right) .
\end{aligned}
$$

This product of the likelihoods ensures the simultaneous and coherent modeling of the HST photometry and ground-based spectroscopy and is a key improvement over the model used in N16. The directed acyclic graph depicting the model is shown in Figure 10.

\subsection{Priors}

We model the prior as separable functions on each of the model parameters. We follow Gelman (2006) and use weakly informative priors on all the parameters of our model. Initial guesses for the intrinsic atmosphere parameters and the spectrum normalization parameter $\left\{T_{\text {eff }}, \log g, d_{L}\right\}$ are

\footnotetext{
${ }^{29}$ http://iancze.github.io/Starfish/
} 


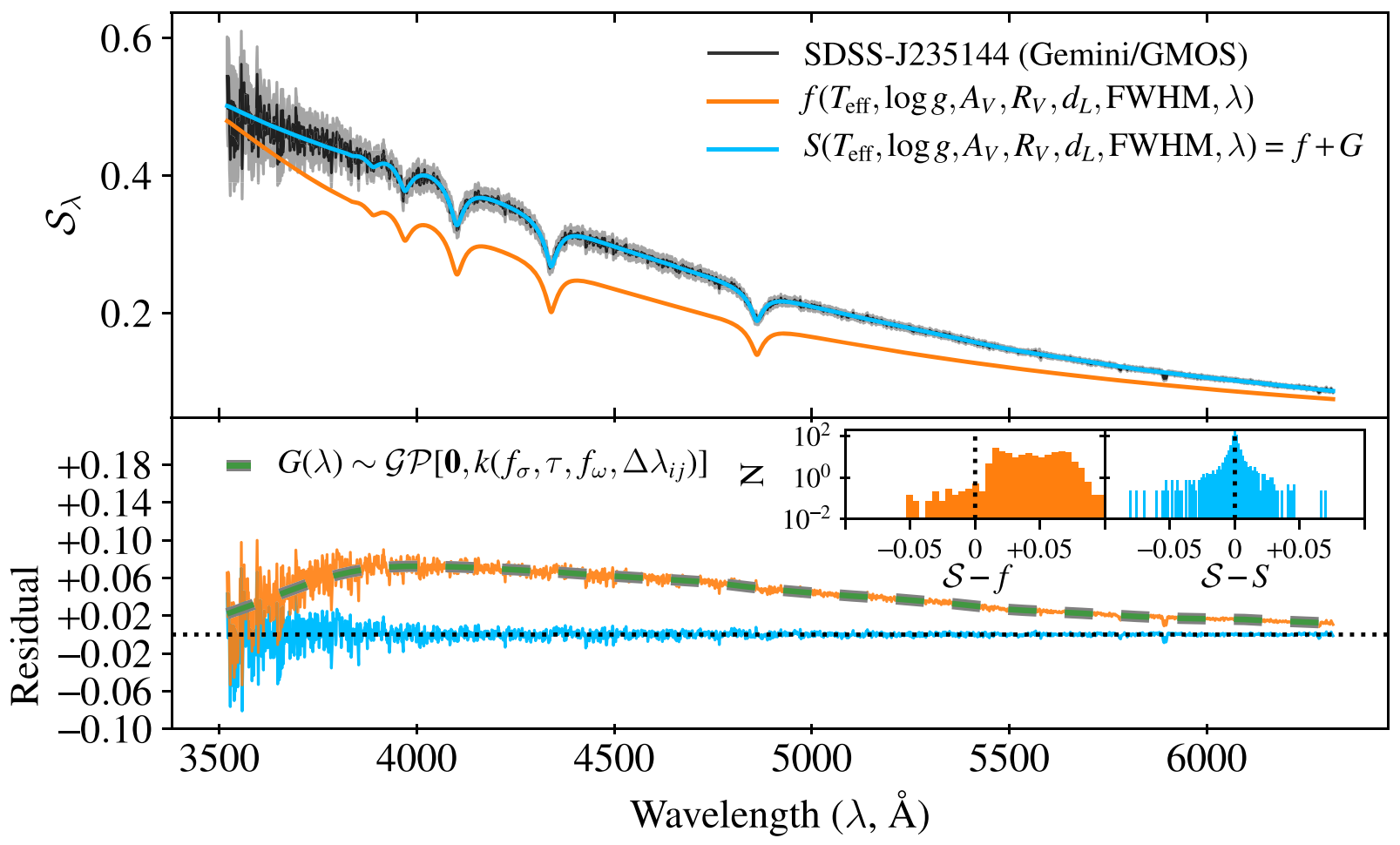

Figure 9. Observed spectrum $\mathcal{S}$ (black, with reported errors in gray) of SDSS J235144 compared to the normalized model spectrum $f\left(T_{\text {eff }}, \log g, A_{V}, R_{V}, d_{L}\right.$, FWHM) (orange). The difference between the model and data is the result of a pronounced miscalibration in the flux from 3500 to $6500 \AA$. The inferred normalized model $f$ does not account for this flux calibration error. We parameterize the flux calibration error with a Gaussian process kernel composed of the sum of a Matérn $3 / 2$ kernel to account for the correlation with wavelength and a white-noise kernel to account for the underestimated error. The corrected spectrum $S$ (blue) agrees well with the observed spectrum $\mathcal{S}$. Without the Gaussian process correction $G$ (the posterior mean is indicated in dashed green in the bottom panel), the residuals to the model (indicated with color corresponding to the model) show significant bias from 0 (indicated by a dotted black line). This bias is readily apparent in the inset log-scaled histograms of the residuals and would lead to a bias in the inferred model parameters if left uncorrected.

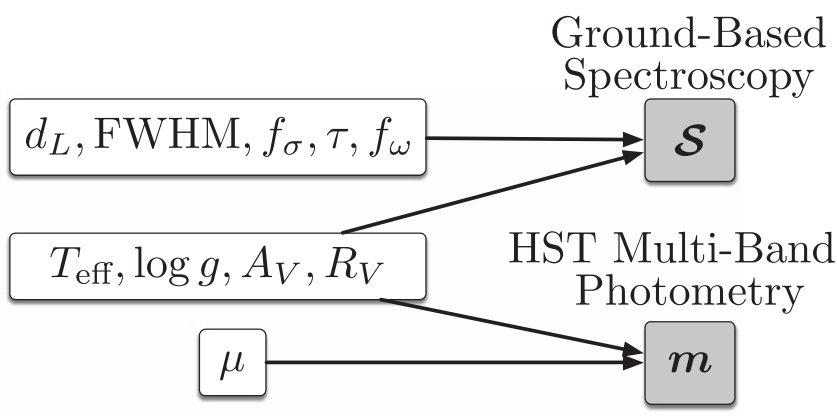

Figure 10. Directed acyclic graph depicting the model for our observations of each DA WD $s-$ the $H S T$ photometry $\left\{m_{s}\right\}$ tied to the three CALSPEC standards (see Section 2.1), and the ground-based spectroscopy $\mathcal{S}$ presented in C19. Clear rounded rectangles denote model parameters, while shaded rounded rectangles denote measurements. The latent (true) properties of the DA WD are described by four parameters: two intrinsic parameters that describe the stellar atmosphere, the effective temperature $T_{\text {eff }}$ and the surface gravity $\log g$, and two extrinsic parameters that describe the line-of-sight Galactic extinction, $A_{V}$ and $R_{V}$. The synthetic model photometry is matched to the observed HST/ WFC 3 photometry using a single normalization constant $\mu$. The observations are modeled as normally distributed about the model magnitudes with variance described by the photometric uncertainty. These five parameters define the latent SED of the DA WD. The spectrum also constrains the intrinsic and extrinsic parameters, but the flux normalization of the spectroscopy is not tied to the normalization of the HST photometry. The normalization of the observed flux of the spectrum is parameterized by $d_{L}$, while the instrumental resolution of the observed spectrum is parameterized by FWHM. Errors in the flux calibration of the observed spectrum are modeled by the posterior mean of a Gaussian process with kernel parameterized by $f_{\sigma}$ and $\tau$ to describe correlated errors with wavelength and $f_{\omega}$ to describe white noise. determined from a least-squares minimization of the model to the observed spectrum $S$ including only the reported uncertainties in the flux of the observed spectrum along the diagonal of the covariance matrix $C$. For this initial fit, $A_{V}$ is fixed to 0 and $R_{V}$ is fixed to 3.1. The FWHM is fixed to an initial input value. The parameter estimates from this initial fit are not accurate enough to model the full data set, but they are sufficient to set weakly informative priors on these parameters. We use normal distributions with variance much larger than the expected error in the parameters to define the marginal priors on $\left\{T_{\text {eff }}, \log g, d_{L}\right\}$ as

$$
\begin{aligned}
P\left(T_{\text {eff }}\right) \propto & N\left(T_{\text {eff }} \mid T_{\text {eff }}^{0}, \sigma=10,000 \mathrm{~K}\right) \\
& \times I_{\left\{16,000 \mathrm{~K} \leqslant T_{\text {eff }} \leqslant 90,000 \mathrm{~K}\right\}}\left(T_{\text {eff }}\right) \\
P(\log g) \propto & N\left(\log g \mid \log g^{0}, \sigma=1 \text { dex }\right) \\
& \times I_{\{7 \leqslant \leqslant \log g \leqslant 9.5\}}(\log g) \\
P\left(d_{L}\right) \propto & N\left(d_{L} \mid d_{L}^{0}, \sigma=1000\right) \times I_{\left\{0<d_{L}<10^{7}\right\}}\left(d_{L}\right),
\end{aligned}
$$

where the indicator function $I_{A}(x)$ returns 1 when $x \in A$ and 0 otherwise, limiting the intrinsic DA WD parameters to the extents of the Tlusty model grid (see Section 6.2), and restricting $d_{L}$ to be strictly greater than 0 .

We set the initial guess for the normalization of the HST/ WFC3 photometry $\mu$ to be the mean difference between the observed photometry $\left\{m_{s}\right\}$ and the synthetic photometry of the 
initial fit SED, determined using Equation (5):

$$
\mu^{0}=\frac{1}{N_{\mathrm{PB}}} \cdot \sum_{\lambda} m_{s, \lambda}-M_{s, \lambda}\left(T_{\mathrm{eff}}^{0}, \log g^{0}, A_{V}^{0}=0, R_{V}^{0}=3.1\right) .
$$

The marginal prior on $\mu$ is

$$
\mu \sim N\left(\mu^{0}, \sigma=10 \mathrm{mag}\right) .
$$

The marginal priors on the extrinsic reddening parameters $A_{V}$ and $R_{V}$ are informed by our knowledge of the Galaxy. We infer these parameters over ranges that are much wider than can be reasonably expected for our low-extinction DA WDs. We use the "glos" distribution, originally introduced in Wood-Vasey et al. (2007) and derived from studies of the line-of-sight extinction to extragalactic sources, as the marginal prior on $A_{V}$. This marginal prior is expressed as the sum of a decaying exponential and a Gaussian distribution for $A_{V} \geqslant 0$ :

$$
\begin{aligned}
P\left(A_{V}\right) \propto & {\left[\frac{1 \mathrm{mag}}{\alpha} \cdot \exp \left(\frac{-A_{V}}{\alpha}\right)+\frac{1 \mathrm{mag}}{\sqrt{2 \pi \sigma^{2}}} \cdot \exp \left(\frac{-A_{V}^{2}}{2 \sigma^{2}}\right)\right] } \\
& \times I_{\left\{0 \leqslant A_{V} \leqslant 2 \mathrm{mag}\right\}}\left(A_{V}\right),
\end{aligned}
$$

where $\alpha=0.4 \mathrm{mag}$ and $\sigma=0.1 \mathrm{mag}$. We can expect the marginal posterior distribution on $A_{V}$ to be much narrower than the "glosz" distribution, as our sources are within the Galaxy, and the extinction is tightly constrained by our multiband $H S T$ photometry. Similarly, we define the marginal prior on $R_{V}$ as

$$
P\left(R_{V}\right) \propto N\left(R_{V} \mid R_{V}=3.1, \sigma=0.18\right) \times I_{\left\{1.7 \leqslant R_{V} \leqslant 5.1\right\}}\left(R_{V}\right),
$$

where $R_{V}=3.1$ is the canonical value of the extinction ratio in our Galaxy for the F99 reddening model, and the standard deviation of the Gaussian is based on the results of Schlafly et al. (2016).

We define the marginal prior on FWHM as a normal distribution centered on the initial guess of the parameter FWHM $^{0}$ supplied with the observed spectrum $\mathcal{S}$. We use $8 \AA$ as the standard deviation of the marginal prior:

$$
\begin{aligned}
P(\mathrm{FWHM}) \propto & N\left(\mathrm{FWHM} \mid \mathrm{FWHM}^{0}, \sigma=8 \AA\right) \\
& \times I_{\{0<\mathrm{FWHM} \leqslant 25 \AA}(\mathrm{FWHM}) .
\end{aligned}
$$

The amplitudes of the component kernels of the Gaussian process are the product of the scale-free amplitudes, $f_{\sigma}$ and $f_{\omega}$, and the median reported uncertainty on the flux of the observed spectrum $\bar{\sigma}_{\mathcal{S}}$. As these parameters describe the noise, we define them to be positive and expect them to be at most $\mathcal{O}(1)$. We therefore define the marginal priors on the scale-free amplitudes as half-Cauchy distributions:

$$
\begin{aligned}
& f_{\sigma} \sim \operatorname{HC}(0, \beta=3) \\
& f_{\omega} \sim \operatorname{HC}(0, \beta=3) .
\end{aligned}
$$

Finally, the marginal prior on the scale of the correlations $\tau$ is defined as

$$
\tau \sim U(500 \AA, 5000 \AA),
$$

appropriate for the reduced spectra presented in $\mathrm{C} 19$, where the splines used for the flux calibration have knots that are more widely spaced than twice the typical $\sim 200 \AA$ width of the $\mathrm{H} \beta$ Balmer feature.

The full prior on the model parameters $\boldsymbol{\Phi}$ is the product of the marginal prior distributions of each of the model parameters:

$$
\begin{aligned}
P(\boldsymbol{\Phi})= & P\left(T_{\mathrm{eff}}\right) \cdot P(\log g) \\
& \times P\left(A_{V}\right) \cdot P\left(R_{V}\right) \\
& \times P(\mu) \\
& \times P\left(d_{L}\right) \cdot P(\mathrm{FWHM}) \\
& \times P\left(f_{\sigma}\right) \cdot P(\tau) \cdot P\left(f_{\omega}\right) .
\end{aligned}
$$

\subsection{Posterior Distribution and Estimation}

The full posterior distribution of the model $\boldsymbol{\Phi}$ given the observations of a DA WD star $s, \mathcal{D}_{s}$, is the product of the likelihood (Equation (27)) and the prior (Equation (36)):

$$
P\left(\boldsymbol{\Phi} \mid \mathcal{D}_{s}\right) \propto P\left(\mathcal{D}_{s} \mid \boldsymbol{\Phi}\right) \cdot P(\boldsymbol{\Phi}) .
$$

We use the apparent magnitudes of our DA WDs tied to the CALSPEC system determined in Section 2.1 and the spectroscopy presented in $\mathrm{C} 19$ without any additional preprocessing. As described in Section 6.10, we perform a fit of the spectrum to obtain initial guesses for the model parameters $\left\{T_{\text {eff }}, \log g, d_{L}, \mu\right\}$. Initial guesses and prior distributions for the remaining parameters either are inputs implicitly provided with the observation $\mathcal{D}$ or can be set without reference to the observations.

We use the Parallel Tempering ensemble Markov Chain Monte Carlo (PTMCMC) algorithm implemented in the emcee python package (Foreman-Mackey et al. 2013). We run four chains at different temperatures, each with 100 walkers. Each walker is initialized to different positions, and we save only every 10 th position of each walker as a step to construct the Markov chain to ensure that the samples are not correlated. Following an initial burn-in of 500 steps, which are discarded, we save a chain with a thinned length of 5000 steps. We use a suite of diagnostic tests for convergence, including visually inspecting the mixing of the chains and inspecting the marginalized 2D joint posterior distributions of the parameters for any artifacts, and verify that the Gelman-Rubin statistic is near unity. Figure 11 presents an example of a DA WD SED inferred with the WDmodel code, combining all the various elements described in Section 6.

While our prior is chosen to be weakly informative, the marginal priors on the extinction parameters $A_{V}$ and $R_{V}$ are physically motivated and therefore merit comparison with the inferred marginal posterior distributions. This posterior predictive test checks whether our inferred parameters are strongly affected by the choice of prior. In Figure 12, we show the inferred marginal posterior distribution of $A_{V}$ for each star compared with the prior. It is evident that the posterior distributions of each star are much narrower than the prior distribution, and our treatment of the "glosz" prior as weakly informative is justified. Our initial fits left $R_{V}$ as a free parameter, but the inferred posterior distribution in $R_{V}$ is not significantly different from the prior. We illustrate this in Figure 13, which compares the recovered mean $R_{V}$ marginal posterior distribution with the prior. This behavior is not surprising, as our DA WDs were selected to be in low line-ofsight extinction regions of our Galaxy, and extinction due to diffuse interstellar dust is well described by a canonical 


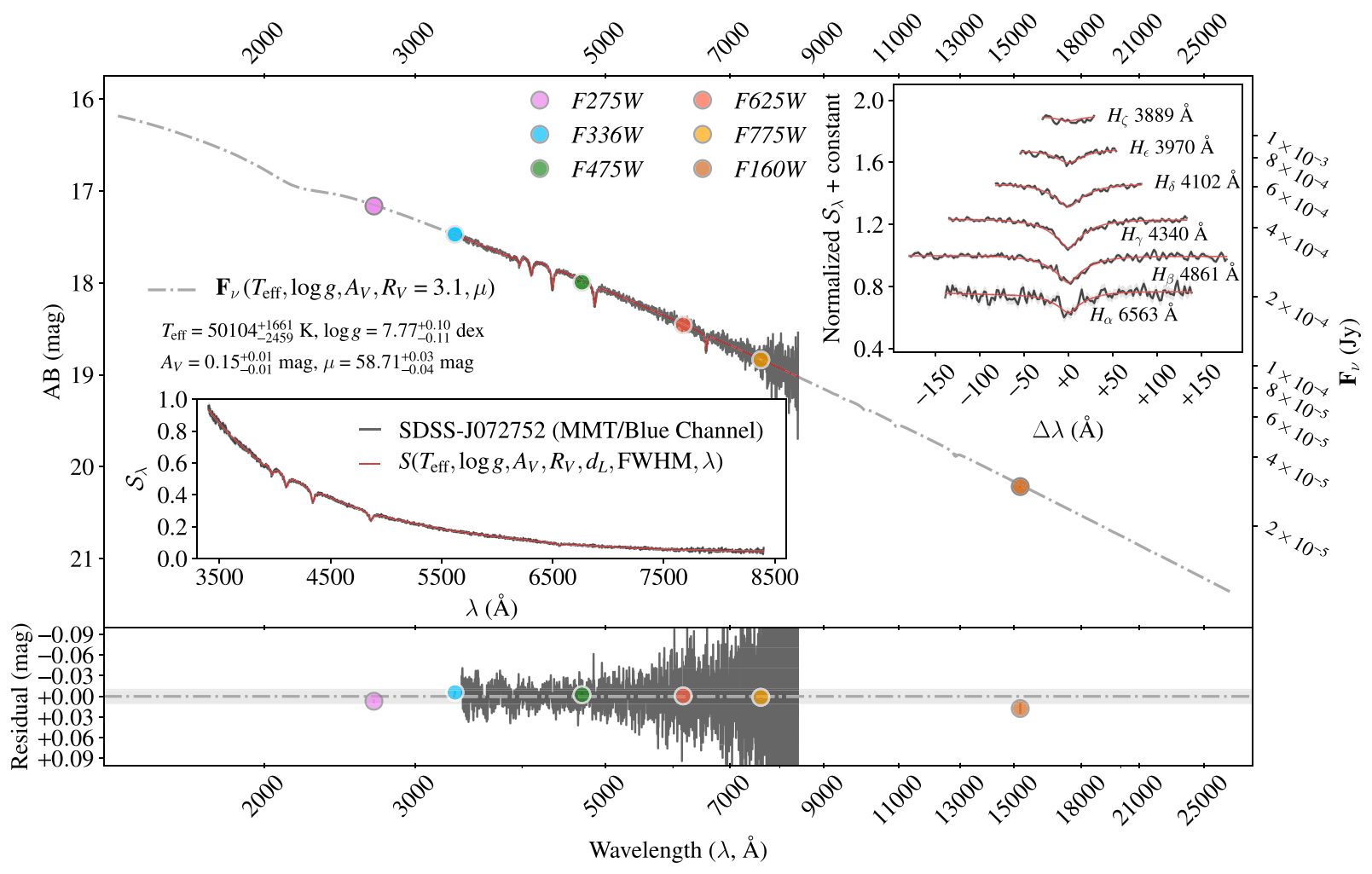

Figure 11. Top: example of an inferred DA WD SED $\boldsymbol{F}_{\nu}$. We model the observed spectrum (lower left inset) and our $H S T$ /WFC3 apparent magnitudes together to infer the parameters of the DA WD SED $\left\{T_{\text {eff }}, \log g, A_{V}, R_{V}=3.1, \mu\right\}$. The observed spectrum $\mathcal{S}_{\lambda}$ (black) and the model of the inferred model spectrum (red) are in excellent agreement. We normalize the continuum of the spectrum to unity and highlight the region around the individual Balmer lines (upper right inset). While both spectrum and model spectrum are plotted, the two are effectively indistinguishable. The photometric normalization parameter $\mu$ is inferred solely from the HST/WFC3 photometry (circle markers, plotted at the effective wavelength). The residuals (bottom) in each of our passband are typically less than $5 \mathrm{mmag}$ for the UVIS channel and $\sim 0.01$ mag for the IR channel. We tie the observed spectrum to the CALSPEC flux scale using the outputs of the WDmodel code. The residuals between the calibrated spectrum and the model are shown in black and are consistent with heteroskedastic white noise.

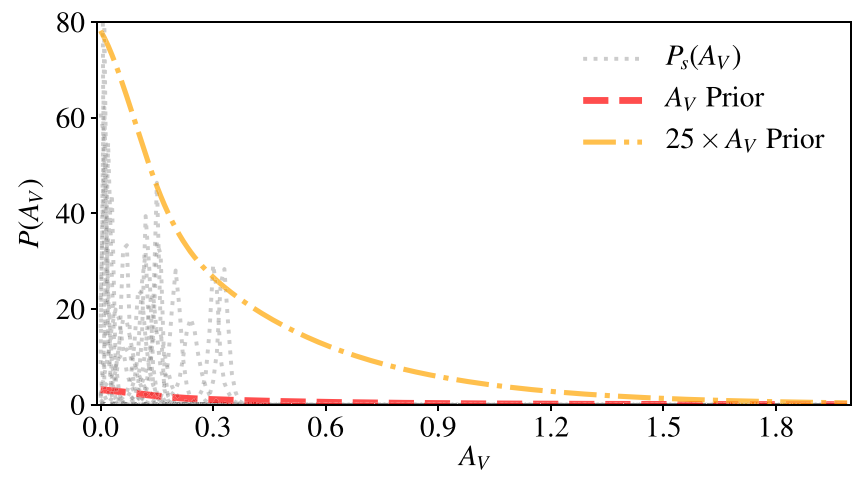

Figure 12. Distributions of inferred $A_{V}$ for each object (gray dotted lines) are much narrower than the "glosz" prior on $A_{V}$ (red dashed line) determined by Wood-Vasey et al. (2007). The shape of the individual marginal posterior distributions is much narrower than the prior (multiplied by a factor of 25 in orange for comparison), and we are justified in treating the "glosz" distribution as a weakly informative prior.

$R_{V}=3.1 \mathrm{~F} 99$ model. We have therefore elected to fix $R_{V}$ to 3.1 for the results presented in this work. This value is appropriate for diffuse interstellar dust outside the plane of our Galaxy, and there is little justification for allowing $R_{V}$ to vary, given that the F99 determination is more precise than the value we can determine from our six-band photometry of each star.

The 1D marginal posterior does not capture the correlations between the parameters. Figure 14 illustrates the 2D joint posterior distributions and 1D marginalized distributions

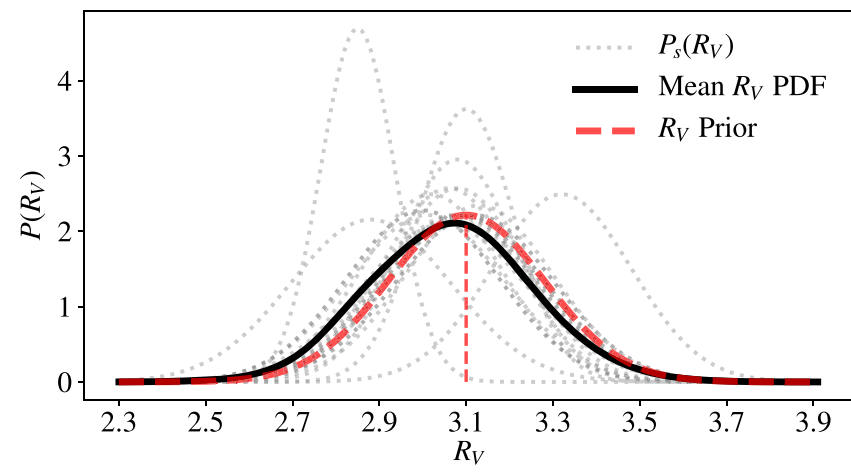

Figure 13. Distributions of inferred $R_{V}$ for each object (gray dotted lines) and the mean distribution of the entire sample (black line) are consistent with the prior on $R_{V}$ centered at 3.1, with a width of 0.18 (red dashed line) determined by Schlafly et al. (2016). This behavior arises because our DA WDs were chosen to be in low-extinction environments, consistent with diffuse interstellar dust. We have fixed $R_{V}=3.1$ for the analysis in this work.

corresponding to the inferred SED in Figure 11. These joint distributions illustrate the strong correlation between $T_{\text {eff }}, A_{V}$ and $\mu$-a model can be made hotter (brighter) and still match our $H S T$ /WFC3 observations if the line-of-sight extinction or the distance to the source is increased. These correlations are weakened by the ground-based spectroscopy, as the temperature cannot be changed without limit because the shape of the Balmer lines and the continuum slope would no longer agree with the observed spectrum. 


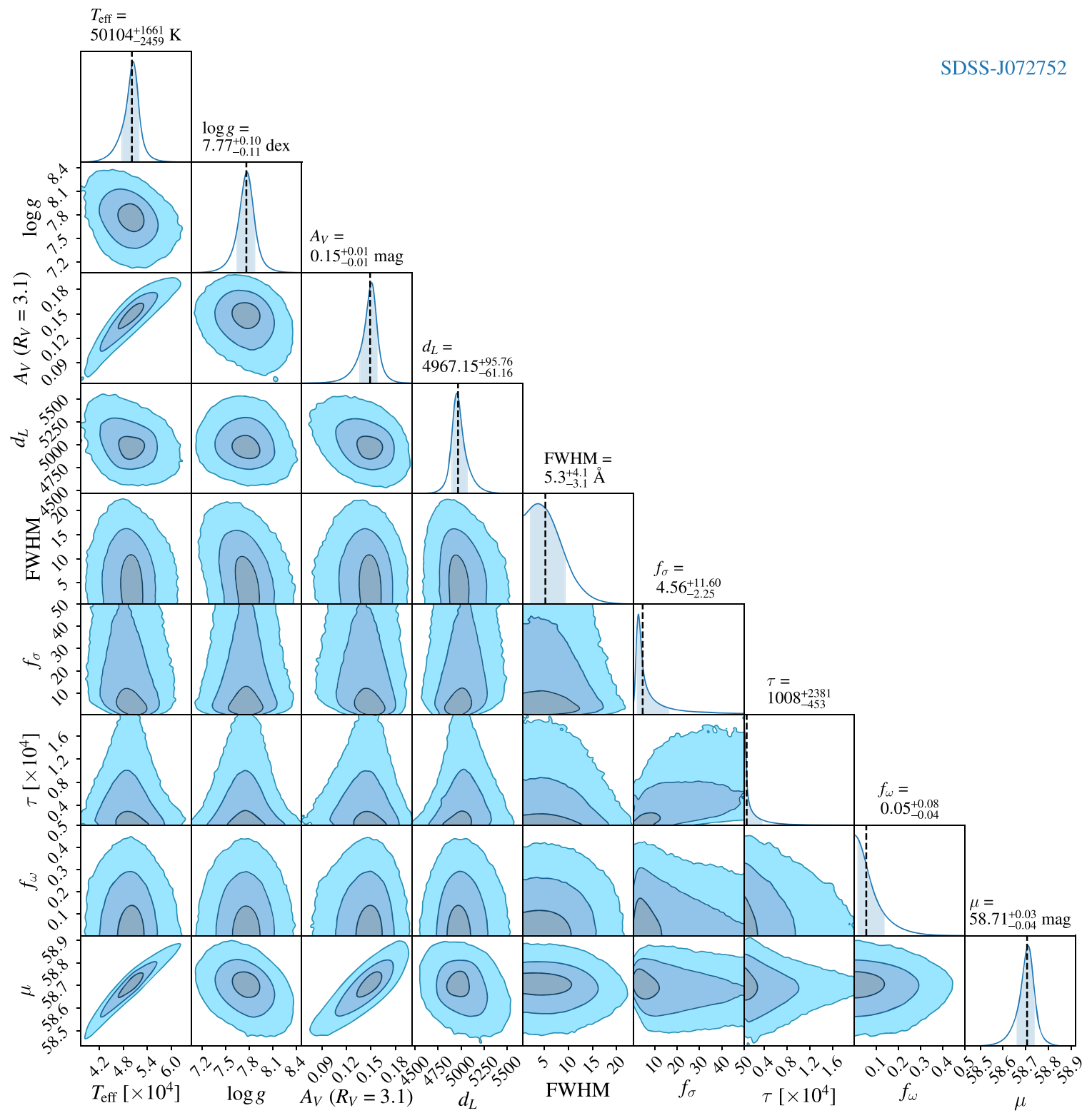

Figure 14. Corner plot for the fit illustrated in Figure 11 showing the 2D joint posterior distributions and 1D marginalized posterior distributions for each of the model parameters. The $1 \sigma, 2 \sigma$, and $3 \sigma$ contours are shown with progressively lighter shading. The 50th percentile of the distribution of each parameter is reported above each 1D marginalization and indicated with a dashed vertical black line.

The correlations between the intrinsic DA WD parameters and the extrinsic parameters were not captured in the analysis presented in N16. As a result of accounting for these correlations, the errors reported on the parameters of our model are larger than in the N16 analysis. Our new WDmodel methodology provides a more principled accounting of the uncertainty associated with the measurements of each object and does not exhibit bias when tested with synthetic spectra. We propagate the uncertainty on the parameters $\left\{T_{\text {eff }}, \log g, A_{V}, \mu\right\}$ to the inferred SED $\boldsymbol{F}$. The SED is independent of the parameters $\left\{d_{L}, \mathrm{FWHM}, f_{\sigma}, \tau, f_{\omega}\right\}$, as these are only used to model the observed spectrum. We have made our WDmodel analysis package ${ }^{30}$ and detailed documentation ${ }^{31}$ public. WDmodel has been successfully used by members of DES to model independently obtained spectra of DA WDs (D. Tucker \& D. Guellidge 2019, private communication).

\footnotetext{
${ }^{30}$ http://github.com/gnarayan/WDmodel/

31 http://wdmodel.rtfd.io
}

\section{Results and Systematics}

We list the model parameters $\left\{T_{\text {eff }}, \log g, A_{V}, \mu\right\}$ describing the SED for each DA WD in our sample in Table 2. The inferred SEDs are tied to the photometric system defined by the three CALSPEC primary standards and are shown in Figure 15. We also present our inferred SEDs of the three CALSPEC standards. We compare our inferred SEDs for the three primary standards to their original CALSPEC SEDs to quantify the systematic differences between our photometric system and the CALSPEC photometric system in Section 7.5.

The model parameters $\left\{d_{L}, \mathrm{FWHM}, f_{\sigma}, \tau, f_{\omega}\right\}$ are specific to each spectrum, are not directly comparable across objects, and have no effect on the inferred SED $\boldsymbol{F}$. A machine-readable table including these parameters for our entire sample of DA WDs, together with plots and posterior samples drawn from the Markov chain for each spectrum, is available through our archive (see footnote 20). We have verified that neither the 
Table 2

Parameters of the DA White Dwarf SEDs Inferred from Spectroscopy and HST Photometry with the WDmodel Code

\begin{tabular}{|c|c|c|c|c|}
\hline Object & $\begin{array}{l}T_{\text {eff }} \\
(\mathrm{K})\end{array}$ & $\begin{array}{c}\log g \\
(\operatorname{dex})\end{array}$ & $\begin{array}{c}A_{V}\left(R_{V}=3.1\right) \\
(\mathrm{mag})\end{array}$ & $\begin{array}{c}\mu \\
(\mathrm{mag})\end{array}$ \\
\hline G191-B2B & $64161_{-776}^{+1126}$ & $7.57_{-0.09}^{+0.08}$ & $0.00_{-0.00}^{+0.00}$ & $52.64_{-0.01}^{+0.02}$ \\
\hline GD 153 & $40087_{-497}^{+827}$ & $7.72_{-0.07}^{+0.07}$ & $0.01_{-0.00}^{+0.01}$ & $53.73_{-0.01}^{+0.02}$ \\
\hline GD 71 & $33012_{-241}^{+417}$ & $7.82_{-0.05}^{+0.04}$ & $0.01_{-0.00}^{+0.01}$ & $53.12_{-0.01}^{+0.02}$ \\
\hline SDSS J010322 & $59061_{-4122}^{+3389}$ & $7.47_{-0.15}^{+0.14}$ & $0.12_{-0.02}^{+0.01}$ & $60.01_{-0.06}^{+0.05}$ \\
\hline SDSS J022817 & $21391_{-608}^{+722}$ & $7.94_{-0.10}^{+0.08}$ & $0.07_{-0.03}^{+0.04}$ & $59.14_{-0.03}^{+0.03}$ \\
\hline SDSS J024854 & $33266_{-797}^{+873}$ & $7.22_{-0.12}^{+0.13}$ & $0.30_{-0.01}^{+0.01}$ & $58.35_{-0.03}^{+0.04}$ \\
\hline SDSS J072752 & $50104_{-2459}^{+1661}$ & $7.77_{-0.11}^{+0.10}$ & $0.15_{-0.01}^{+0.01}$ & $58.71_{-0.04}^{+0.03}$ \\
\hline SDSS J081508 & $34735_{-1109}^{+1709}$ & $7.20_{-0.10}^{+0.11}$ & $0.07_{-0.02}^{+0.03}$ & $60.06_{-0.04}^{+0.06}$ \\
\hline SDSS J102430 & $36691_{-1483}^{+1666}$ & $7.54_{-0.22}^{+0.28}$ & $0.24_{-0.02}^{+0.02}$ & $59.13_{-0.05}^{+0.05}$ \\
\hline SDSS J111059 & $46298_{-2339}^{+1839}$ & $7.85_{-0.12}^{+0.12}$ & $0.15_{-0.01}^{+0.01}$ & $58.48_{-0.05}^{+0.03}$ \\
\hline SDSS J111127 & $59422_{-2257}^{+2105}$ & $7.76_{-0.13}^{+0.12}$ & $0.03_{-0.01}^{+0.01}$ & $59.45_{-0.03}^{+0.03}$ \\
\hline SDSS J120650 & $23434_{-408}^{+456}$ & $7.94_{-0.05}^{+0.05}$ & $0.04_{-0.02}^{+0.02}$ & $58.21_{-0.02}^{+0.02}$ \\
\hline SDSS J121405 & $33750_{-465}^{+707}$ & $7.96_{-0.10}^{+0.08}$ & $0.01_{-0.01}^{+0.01}$ & $58.11_{-0.02}^{+0.03}$ \\
\hline SDSS J130234 & $40028_{-1238}^{+1377}$ & $7.94_{-0.08}^{+0.08}$ & $0.06_{-0.01}^{+0.01}$ & $57.59_{-0.03}^{+0.03}$ \\
\hline SDSS J131445 & $43670_{-2035}^{+2812}$ & $7.65_{-0.14}^{+0.18}$ & $0.11_{-0.02}^{+0.02}$ & $59.72_{-0.04}^{+0.05}$ \\
\hline SDSS J151421 & $28768_{-300}^{+297}$ & $7.89_{-0.04}^{+0.04}$ & $0.12_{-0.01}^{+0.01}$ & $55.58_{-0.01}^{+0.01}$ \\
\hline SDSS J155745 & $56760_{-2042}^{+1787}$ & $7.69_{-0.12}^{+0.11}$ & $0.02_{-0.01}^{+0.01}$ & $58.46_{-0.03}^{+0.02}$ \\
\hline SDSS J163800 & $57181_{-3931}^{+4238}$ & $7.63_{-0.24}^{+0.25}$ & $0.20_{-0.02}^{+0.01}$ & $59.62_{-0.06}^{+0.06}$ \\
\hline SDSS J181424 & $30806_{-258}^{+325}$ & $7.88_{-0.05}^{+0.05}$ & $0.01_{-0.01}^{+0.01}$ & $56.71_{-0.01}^{+0.02}$ \\
\hline SDSS J210150 & $29062_{-536}^{+516}$ & $7.85_{-0.08}^{+0.09}$ & $0.14_{-0.02}^{+0.02}$ & $58.54_{-0.02}^{+0.02}$ \\
\hline SDSS J232941 & $21044_{-500}^{+445}$ & $7.96_{-0.07}^{+0.07}$ & $0.15_{-0.03}^{+0.03}$ & $57.35_{-0.02}^{+0.02}$ \\
\hline SDSS J235144 & $41058_{-1752}^{+1993}$ & $7.99_{-0.17}^{+0.15}$ & $0.33_{-0.01}^{+0.01}$ & $58.35_{-0.04}^{+0.04}$ \\
\hline
\end{tabular}

Note. Parameters are reported as the median of the marginal posterior distributions. The $\pm 34 \%$ interval about the median is reported as a superscript and subscript, respectively. For objects with multiple spectra, we provide the parameters of the result with the highest log-likelihood.

photometric residuals in any passband nor the spectroscopic residuals correlate with any of the inferred model parameters, indicating that the posterior distribution is well sampled and there are no unmodeled correlations. The residuals between the spectra and the model are consistent with white noise.

The photometric residuals for each DA WD in each passband are presented in Table 3 and are shown in Figure 16. The residuals of the three CALSPEC standards are also shown but not used in the computation of summary statistics. Our analysis extends the set of DA WDs with SED models that predict observed fluxes to better than $1 \%$ from the three CALSPEC standards to 22 objects. This analysis also extends the dynamic range spanned by the stars from $\sim 1.5$ to $\sim 7.5 \mathrm{mag}$. The standard deviation of the residuals of our DA WDs across all passbands is 6 mmag.

\subsection{Evaluating Sources of Systematic Errors}

We must evaluate any correlated bias in the inference, leading to all the SED models differing systematically from the truth. While the agreement between model and data is better than $1 \%$, it is critical to control these systematic effects, as errors in the spectrophotometric standards can propagate across wide-field surveys. It is likely that some of these systematic errors already affect our inference. A conspicuous feature of the photometric residuals in Figure 16 is that the model and data disagree at the few millimagnitude level in F160W in the sense that the model overestimates the flux in the NIR. This disagreement is particularly evident in comparison to the WFC3/UVIS passbands. The mean residual of our DA WD in each band $\mu$ is less than 4 mmag in all bands except F160W. Excluding F160W reduces the standard deviation of the residuals to $4 \mathrm{mmag}$. The mean residual in each passband is consistent with 0 except in $\mathrm{F} 160 \mathrm{~W}$, where there is a $9 \mathrm{mmag}$ bias in the sense of the observed magnitudes being too faint relative to the model prediction. In order to mitigate these systematic errors, we must identify their sources and estimate their impact. There are several elements of our analysis that are common to all objects (see Figure 1) and therefore potential sources of systematic error in our SEDs:

1. The DA WD atmosphere model grid.

2. The shape of the reddening law of wavelength.

3 . The shape of the passband response functions.

4. The overall flux normalization or zero-points.

5. The linearity of the HST/WFC3 detectors.

We evaluate the effect of each on the SED models in the following sections, and we summarize their impact in Section 7.8 .

\subsection{Errors in the DA WD Model Grid}

To estimate any error in the SEDs caused by an error in the DA WD model atmosphere grid, we considered a different atmosphere grid provided by one of us (I. Hubeny). This new grid extends to $30 \mu \mathrm{m}$ and is based on Tlusty v205 (Hubeny \& Lanz 2017). The new grid incorporates Bracket and Pfund series line profiles and improves the smoothing of the Lyman and Balmer pseudocontinua. Numerically, the NLTE models were constructed using 30,000 internal frequency points to model the discretized mean intensity of radiation, while the original grid used in this work (see Section 6.2) used 5000. The emergent spectra are constructed with resolution $R=5000$. Finally, the spacing in $\log g$ was reduced to 0.25 dex from the 0.5 dex spacing used in this work. We found no difference between the new grid and the grid we use in this work. Consequently, the intrinsic parameters and inferred SEDs are in very close agreement.

We also compared our Tlusty model grid versus models generated from the Tübingen NLTE Model Atmosphere Package (TMAP; Rauch 2016) grid at the same model parameters $\left\{T_{\text {eff }}, \log g\right\}$. The mean difference between the model atmospheres at the same model parameters is irrelevant, as this would be absorbed into the overall flux normalization parameter $\mu$ in Section 6. We found that the residual differences about the mean offset are 1-2 mmag, significantly smaller than the discrepancy in F160W we are seeking to explain. We note that comparison of the two grids at the same values of the intrinsic parameters is a bound on the worst-case error, and N16 demonstrated that the best-matching model for any TMAP atmosphere from the Tlusty grid has a slightly different $\left\{T_{\text {eff }}, \log g\right\}$. It is possible that there is a commonmode error in the shape of the continuum of all the DA WD model grids. However, given that the existing residuals between our SED models and data are consistent with white noise in the WFC3/UVIS passbands, any such error must be $<1$ mmag.

All of our DA WD stars have very similar colors, and any error in the grid would likely cause a nearly constant offset in 


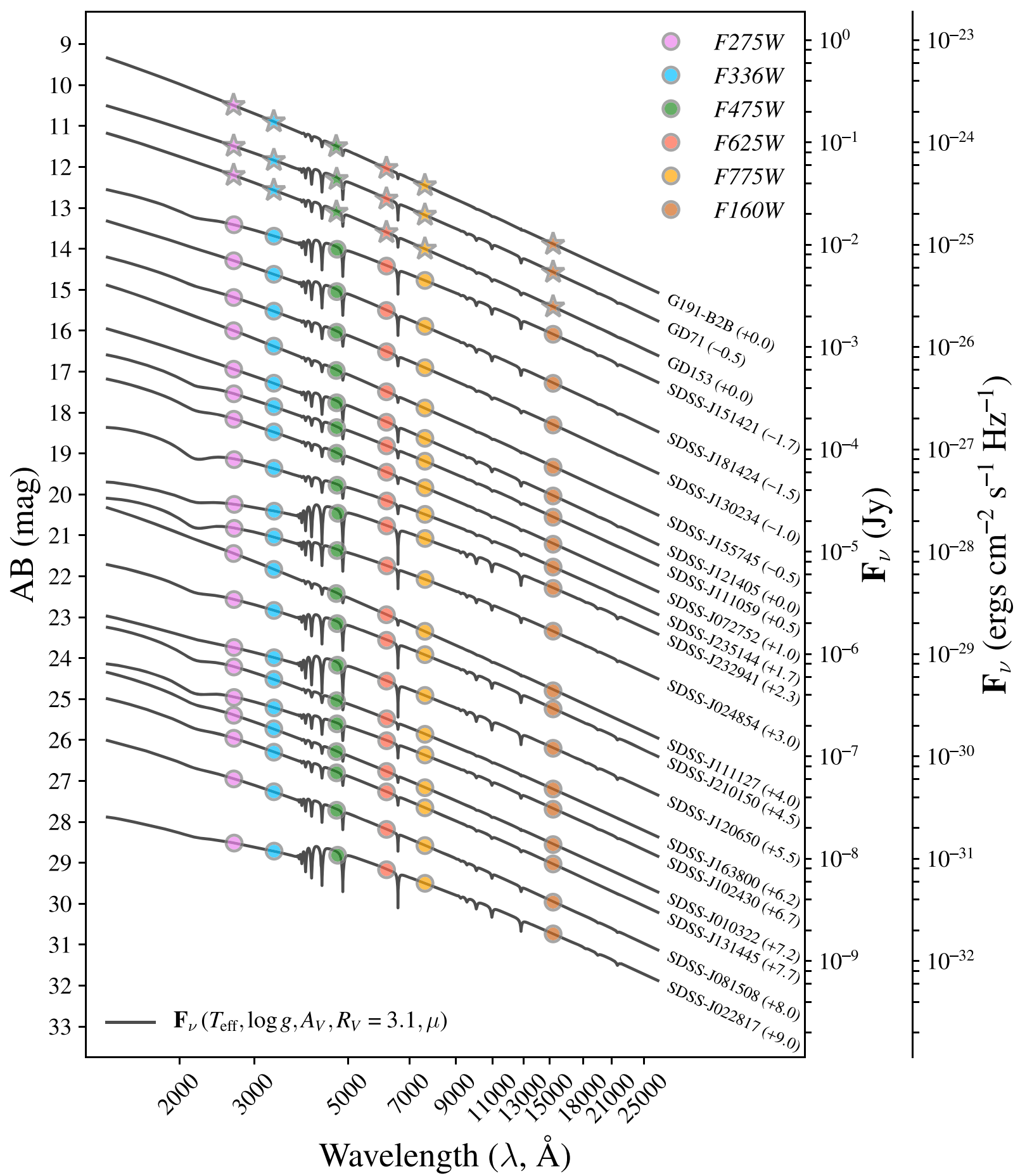

Figure 15. Sequence of calibrated SEDs of our DA WDs shown in AB magnitudes. The SEDs are spaced vertically for clarity by a magnitude offset indicated parenthetically in the label with the object name. The axes on the right specify the SED per unit frequency $\boldsymbol{F}_{\nu}$, in units of janskys and erg $\mathrm{cm}^{-2} \mathrm{~s}^{-1} \mathrm{~Hz}^{-1}$. The observed $H S T$ /WFC3 photometries presented in Table 1 are shown with colored markers (stars for the three CALSPEC primary standards, circles for our program stars) on each SED at the effective wavelength of each source through each passband. The uncertainties on the photometry and the inferred SEDs are smaller than the markers and the lines, respectively. The synthetic model magnitudes and residuals between the observations and the synthetic model magnitudes are presented in Table 3 and Figure 16.

the residuals. In particular, the atmosphere of DA WDs is largely dominated by the Rayleigh-Jeans tail at NIR wavelengths and is nearly flat and featureless. We do not see any constant offsets in our photometric residuals. It would be a complex proposition to modify the grid in such a way as to cause the residuals to be consistent with zero for some objects but have significant residuals for others. Nevertheless, we can also rule out errors in the model atmospheres that are prevalent in some regions of parameter space but not others, as the coefficient of correlation between residuals in F160W and either of the DA WD intrinsic parameters, $T_{\text {eff }}$ and $\log g$, is consistent with 0 . Errors in the DA WD model grid cannot explain the discrepancy in F160W, and the mean of the residuals in the WFC3/UVIS passbands is $\sim 1 \mathrm{mmag}$. Any 


\begin{tabular}{|c|c|c|c|c|c|c|c|c|c|c|c|c|}
\hline \multirow[b]{2}{*}{ Object } & \multicolumn{11}{|c|}{$\begin{array}{c}\text { Table } \mathbf{3} \\
\text { Model AB Magnitudes and Residuals for the Network of DA White Dwarfs and CALSPEC Primary Standards }\end{array}$} & \multirow[b]{2}{*}{$\mathrm{rF} 160 \mathrm{~W}$} \\
\hline & \multicolumn{11}{|c|}{$(\mathrm{AB}$ mag $)$} & \\
\hline G191-B2B & 10.4903 & 0.0000 & 10.8891 & +0.0011 & 11.5023 & -0.0035 & 12.0326 & -0.0020 & 12.4493 & +0.0021 & 13.8800 & +0.0052 \\
\hline GD 153 & 12.2037 & -0.0021 & 12.5659 & +0.0020 & 13.0978 & +0.0020 & 13.5977 & -0.0001 & 14.0029 & -0.0012 & 15.4138 & +0.0001 \\
\hline GD 71 & 11.9910 & -0.0022 & 12.3345 & +0.0015 & 12.7979 & +0.0009 & 13.2770 & +0.0020 & 13.6743 & -0.0023 & 15.0675 & +0.0001 \\
\hline SDSS J010322 & 18.1890 & +0.0062 & 18.5340 & -0.0072 & 19.0877 & -0.0044 & 19.5716 & -0.0030 & 19.9602 & +0.0046 & 21.3401 & +0.0150 \\
\hline SDSS J022817 & 19.5174 & +0.0009 & 19.7176 & -0.0024 & 19.8132 & +0.0019 & 20.1689 & +0.0001 & 20.5025 & -0.0011 & 21.7383 & -0.0013 \\
\hline SDSS J024854 & 17.8272 & +0.0014 & 18.0441 & -0.0041 & 18.3704 & -0.0008 & 18.7464 & -0.0004 & 19.0760 & +0.0012 & 20.3427 & -0.0027 \\
\hline SDSS J072752 & 17.1566 & +0.0070 & 17.4772 & -0.0058 & 17.9956 & -0.0024 & 18.4575 & -0.0008 & 18.8357 & +0.0013 & 20.1990 & +0.0176 \\
\hline SDSS J081508 & 18.9465 & +0.0040 & 19.2684 & -0.0048 & 19.7178 & -0.0016 & 20.1877 & -0.0039 & 20.5746 & +0.0048 & 21.9462 & +0.0155 \\
\hline SDSS J102430 & 18.2532 & +0.0074 & 18.5085 & +0.0058 & 18.9113 & -0.0072 & 19.3182 & -0.0008 & 19.6667 & -0.0017 & 20.9730 & +0.0176 \\
\hline SDSS J111059 & 17.0427 & -0.0020 & 17.3561 & -0.0017 & 17.8600 & +0.0069 & 18.3152 & -0.0017 & 18.6908 & -0.0021 & 20.0499 & +0.0066 \\
\hline SDSS J111127 & 17.4422 & +0.0007 & 17.8262 & +0.0036 & 18.4209 & -0.0004 & 18.9378 & +0.0012 & 19.3476 & -0.0035 & 20.7676 & +0.0299 \\
\hline SDSS J120650 & 18.2418 & -0.0022 & 18.4852 & +0.0036 & 18.6735 & -0.0016 & 19.0620 & -0.0019 & 19.4136 & -0.0025 & 20.6914 & +0.0113 \\
\hline SDSS J121405 & 16.9400 & 0.0000 & 17.2841 & -0.0014 & 17.7585 & +0.0021 & 18.2366 & -0.0004 & 18.6335 & -0.0043 & 20.0281 & +0.0098 \\
\hline SDSS J130234 & 16.1853 & +0.0026 & 16.5244 & -0.0028 & 17.0358 & +0.0006 & 17.5128 & +0.0012 & 17.9048 & -0.0012 & 19.2938 & +0.0094 \\
\hline SDSS J131445 & 18.2614 & -0.0037 & 18.5900 & +0.0069 & 19.1000 & +0.0018 & 19.5705 & -0.0037 & 19.9557 & -0.0004 & 21.3307 & -0.0023 \\
\hline SDSS J151421 & 15.1155 & -0.0055 & 15.3860 & +0.0046 & 15.7075 & +0.0015 & 16.1165 & +0.0037 & 16.4748 & -0.0036 & 17.7867 & +0.0003 \\
\hline SDSS J155745 & 16.4977 & +0.0022 & 16.8803 & -0.0036 & 17.4700 & +0.0002 & 17.9864 & +0.0054 & 18.3962 & -0.0082 & 19.8159 & +0.0183 \\
\hline SDSS J163800 & 18.0125 & +0.0033 & 18.3199 & -0.0022 & 18.8346 & +0.0053 & 19.2846 & -0.0037 & 19.6531 & +0.0073 & 20.9981 & -0.0019 \\
\hline SDSS J181424 & 15.7902 & +0.0012 & 16.1215 & -0.0002 & 16.5413 & +0.0028 & 17.0061 & -0.0004 & 17.3971 & -0.0044 & 18.7743 & +0.0113 \\
\hline SDSS J210150 & 18.0669 & +0.0013 & 18.3337 & +0.0007 & 18.6560 & 0.0000 & 19.0629 & +0.0008 & 19.4193 & -0.0053 & 20.7283 & +0.0113 \\
\hline SDSS J232941 & 17.9449 & -0.0015 & 18.1055 & +0.0035 & 18.1524 & +0.0083 & 18.4735 & -0.0038 & 18.7859 & -0.0107 & 19.9826 & +0.0123 \\
\hline SDSS J235144 & 17.4394 & +0.0099 & 17.6703 & -0.0084 & 18.0766 & -0.0014 & 18.4569 & +0.0026 & 18.7887 & -0.0018 & 20.0699 & +0.0048 \\
\hline
\end{tabular}

Model AB Magnitudes and Residuals for the Network of DA White Dwarfs and CALSPEC Primary Standards

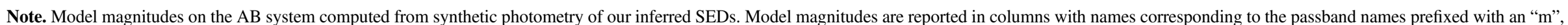
while the differences between the observed magnitudes reported in Table 1 and model magnitudes are reported in columns prefixed with an "r." All quantities are rounded to $1 / 10$ of a millimagnitude. 


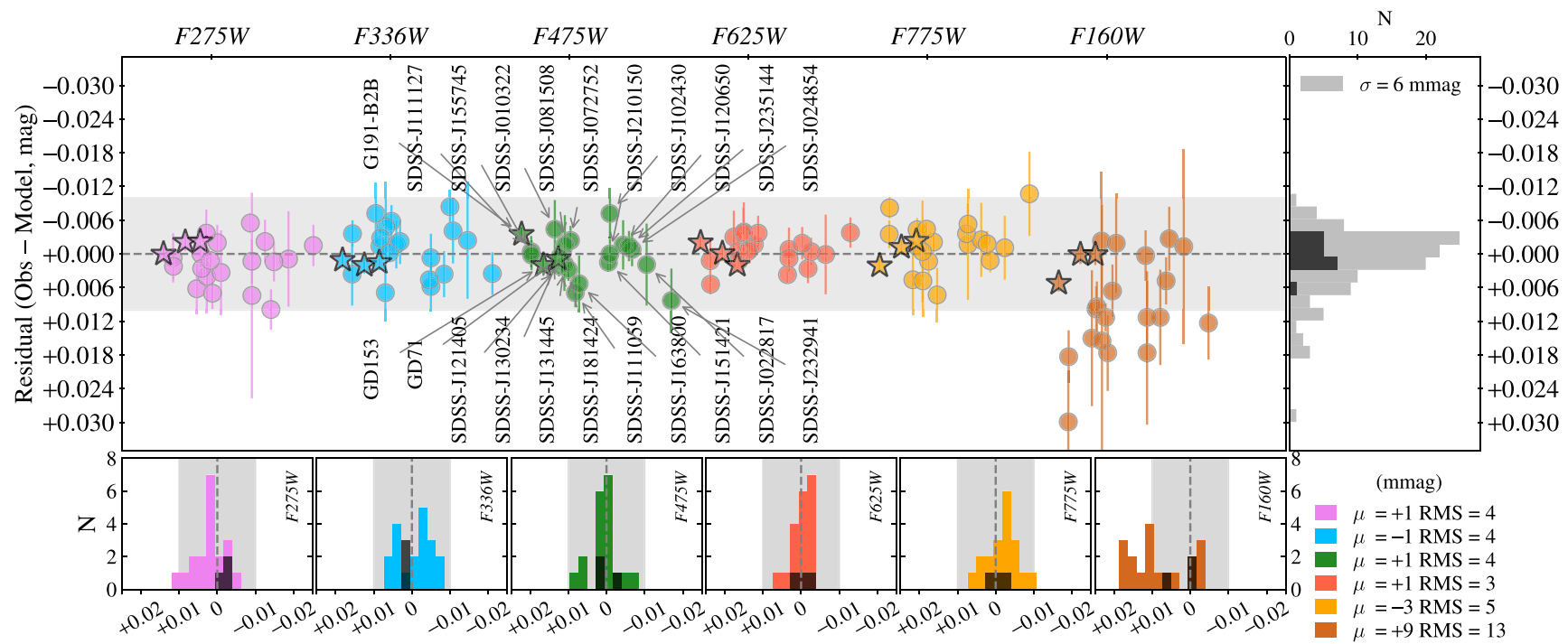

Figure 16. Left, top row: photometric residuals (in the sense of observed magnitude-model magnitude) for our network of DA WD stars (circles) and CALSPEC primary standards (stars, not included in the computation of any summary statistics) in each passband. Objects are labeled in the F475W passband, and their relative horizontal position is set by an amount proportional to their $g-r$ color and is the same across all passbands. The light-gray region indicates a standard deviation of 0.1 mag. Right, top row: the distribution of residuals of our network DA WDs across all passbands has a standard deviation of 6 mmag. Excluding F160W reduces this standard deviation to $\sim 3 \mathrm{mmag}$. Bottom row: histogram of residuals in each passband with the mean residual and rms indicated in the legend. As above, the light-gray region indicates a standard deviation of $0.1 \mathrm{mag}$. The three primary standards are indicated in black in all the histograms. The mean residual in each band $\mu$ is less than 5 mmag in all bands except F160W. The rms and standard deviation of the DA WDs are much larger in F160W than in any of the optical passbands.

systematic error in the DA WD model grid must be below this level.

\subsection{Errors in the Reddening Law}

As noted previously, we found no significant difference when using the O'Donnell (1994) reddening instead of F99. Additionally, we considered a custom reddening law defined for $R_{V}=3.1$ constructed by J. Holberg from Gaia DR2 observations and included with the WDmodel code. This too did not cause a significant difference in the photometric residuals in any passband. Allowing $R_{V}$ to vary from 3.1 causes the weighted mean of the residuals in F160W to decrease from 9 to $7 \mathrm{mmag}$ - an insignificant change when accounting for the addition of the extra free parameter. Additionally, the difference between $R_{V}=3.1$ and the true value of $R_{V}$ for each object cannot be correlated, as our program stars are spread across the sky. Therefore, any error in the SEDs induced by fixing $R_{V}$ to 3.1 is only likely to cause dispersion, rather than a systematic bias in the SEDs.

It is unlikely that any change to the reddening law can resolve this discrepancy in F160W, as dust causes less extinction at these wavelengths relative to the optical $\left(R_{H}=0.464\right)$. Resolving a mean residual difference in F160W of $9 \mathrm{mmag}$ with a change in reddening requires $R_{H} \sim 1.5$ without changing the coefficient of the reddening law in any other band. Such a change would be completely unphysical, as there is no reason to expect the dust along the line of sight to DA WDs to extinguish the flux more strongly than along other lines of sight in the Galaxy. Any error in the reddening applied to the model is extremely unlikely to be the source of the bias.

\subsection{Errors in the Passband Model}

We model the passband response using the pysynphot files provided by STScI (see footnote 21). The response of the filters was determined pre-flight. While there are periodic adjustments to the overall normalization of the components, these are achromatic. The GRW70 data presented in Section 4.1 have slightly different slopes in each passband. This indicates that the shape of the response is evolving with time. This error is likely to be irreducible. When designing our Cycle 22 observing program, we determined that it was not possible to have both the WFC3 grism and passbands in the optical path at the same time, which would allow an in-flight determination of the throughput. The WFC3 flight spares have not been subjected to the same conditions as the filters on HST, and using their transmission as a proxy is likely to introduce new systematic errors.

Recognizing that a systematic difference between the passband model and the true passband response constitutes a systematic floor for our experiment, we sought to mitigate it in designing our observing program. The photometric zero-points inferred in Section 4 are largely determined by the difference between the synthetic and instrumental photometry of the three CALSPEC primary standards. Any error in the passband model would lead to an error in the synthetic magnitudes and therefore the zero-points. These zero-points are applied to the measured instrumental photometry of our program stars, leading to an error in apparent magnitudes. If the SEDs of our program standards differed significantly from those of the CALSPEC primary standards, there would be a systematic trend in the residuals with color. As the primary standards are also DA WDs and have very similar colors to our program stars, we reduce the impact of any error in the specification of the passband response.

Moreover, the GRW70 data constrain the maximum change in the response to be at most 1-2 mmag per year. Much of this change is dominated by the decrease in sensitivity, rather than the change in passband shape. An error in the passband shape is likely to have a $<1$ mmag impact on our SEDs. We emphasize that mitigating this error is not the same as measuring the passband response accurately, and in particular for sources with significantly different SEDs than the primary standards, we can 
expect a trend in the residuals as a function of color. An observational campaign to measure all the CALSPEC standards with all the HST/WFC3 passbands could, in principle, be used to determine a correction to each response. Given the constraints of the GRW70 data, such a campaign would have a limited impact on the overall accuracy of our experiment.

A systematic error in the passband model cannot explain the discrepancy in F160W. Any adjustment to the shape of the F160W passband produces an almost common-mode bias affecting all objects equally, as the spectral flux density of DA WD stars is nearly flat per unit wavelength across this passband. This is markedly different than the disagreement between the observations and model in our analysis, where some objects have residuals consistent with 0 , while others show significant offsets. There is no change that can be applied to reduce the residual for the largest outliers that does not increase the residual by an almost identical amount for objects where the model agrees with the observations. We find no significant trend in the residual with MJD or with cycle number that might indicate an evolution in the passband response, and we rule out passband shape adjustments as an explanation for the discrepancy in F160W.

\subsection{Errors in the Overall Flux Normalization}

The residuals presented in Table 3 and Figure 16 quantify the level of agreement between our measured HST/WFC3 photometry and our inferred SEDs. However, any error in the passband zero-points derived in Section 4 would propagate to all of the apparent magnitudes and therefore all of the inferred SEDs. Such a systematic error in the passband zero-points can be considered a difference between the photometric system defined by our inferred SEDs for the DA WD stars and the photometric system defined by the CALSPEC SEDs of the three primary standards.

Such a difference could be induced because the measurement chain employed by the CALSPEC team differs from that employed in $\mathrm{C} 19$ and this work. While we measure the primary standards using the same $H S T /$ WFC3 instrumental configuration as our program stars, the original B14 CALSPEC SEDs are determined from $H S T / S T I S$ spectroscopy and HST/ACS photometry. As noted previously, our zero-points are effectively determined by the difference between the CALSPEC synthetic magnitudes and our measured HST/WFC3 instrumental photometry. Any systematic error in the flux ratios between the CALSPEC SEDs of the three primary standards would propagate into an error in our zero-points.

Had we obtained our observations with the same instrumentation as the primary standards, a purely differential measurement would have sufficed to calibrate measured count rates with respect to the primary standards. We would not have needed to use the CALSPEC SED models and could have determined fluxes relative to the primary standard observations. With such a measurement chain, a single overall achromatic zero-point suffices to set the flux scale. Such an approach avoids any systematic errors introduced by tying to the CALSPEC SED models. Unfortunately, despite the conceptual attractiveness of a differential measurement, using the same measurement chain for the DA WDs and the CALSPEC primary standards was never a practical option. The WFC channel of HST/ACS has low QE below $4000 \AA$ and above $1 \mu \mathrm{m}$, and observations at UV and NIR wavelengths are critical to determining the line-of-sight extinction, while exposure times with HST/STIS are prohibitive for our faint standards.

We quantify the systematic difference to the CALSPEC photometric system from our inferred SEDs for the three primary standards in Figure 17, as well as from our measured HST/WFC3 photometry in Table 4-the first approach incorporates all the systematics from an end-to-end analysis, while the second is independent of the differences between the model atmosphere grids. The residuals between our observed HST/WFC3 observations and the synthetic CALSPEC magnitudes in Table 4 are completely negligible for GD 153 and GD191-B2B but have a mean of $\sim 5$ mmag for GD 71. This indicates that there is some tension between the flux ratios of the primary standards measured from the HST/WFC3 images and the flux ratios defined by their CALSPEC SEDs.

With our existing data, we cannot distinguish whether the underlying source of this tension arises from the measurement (i.e., a bias in our HST/WFC3 data) or the model (i.e., an error in the CALSPEC SEDs). As described in Section 3.5, we elected to read out the C512C subarray of WFC3/UVIS in Cycle 22, when we began monitoring the primary standards. The small images are unsuitable for artificial star injection tests, which would allow us to determine whether there is a weak bias in the recovered photometry. Given that the repeatability of $H S T / W F C 3$ described in Section 4.1 and illustrated in Figure 4 is $5-8 \mathrm{mmag}$, and that observations in all passbands were obtained in the same orbit, it is possible that the nonzero offset of GD 71 is simply the result of a correlated statistical fluctuation. At the same time, we do not have observations of the remaining 90 CALSPEC standards with $H S T /$ WFC3 to determine whether the CALSPEC GD 71 model itself is inconsistent with the observations. The nonzero residuals for GD 71 drive the weighted mean difference in the zero-point between our photometric system and the CALSPEC photometric system, but these biases are small (1-2 mmag).

The difference between our apparent magnitudes and the CALSPEC synthetic magnitudes is the dominant contribution to the difference between our inferred SEDs and the original CALSPEC SED models. The shape differences about the median offset are dominated by differences in the line profiles. These differences arise from the different Stark broadening prescriptions used by our Tlusty grid and the TMAP models employed by CALSPEC, but they have negligible impact on broadband photometry. G191-B2B exhibits the largest difference in shape correlated with wavelength. The CALSPEC SED of G191-B2B has $A_{V}=0.0016 \mathrm{mag}$, whereas we infer an extinction of less than $1 \mathrm{mmag}$ from our HST/WFC3 photometry and MMT/Blue Channel spectroscopy of this object. This difference in reddening dominates the overall shape of the residual. The CALSPEC G191-B2B SED includes metal lines (Rauch et al. 2013), while our SED uses a purehydrogen atmosphere. The difference is evident in the residual between the two SEDs in the UV. However, as the median difference between our inferred SEDs is dominated by the difference in the observed and synthetic HST/WFC3 photometry, any systematic must be dominated by the difference in the measurement chains, rather than the model grids or the zero-points.

Conservatively, we adopt $4 \mathrm{mmag}$, corresponding to the median error on GD 71, as our estimate of the systematic error in the overall flux normalization of the SED. Observers using our network of stars to compute synthetic magnitudes derived 


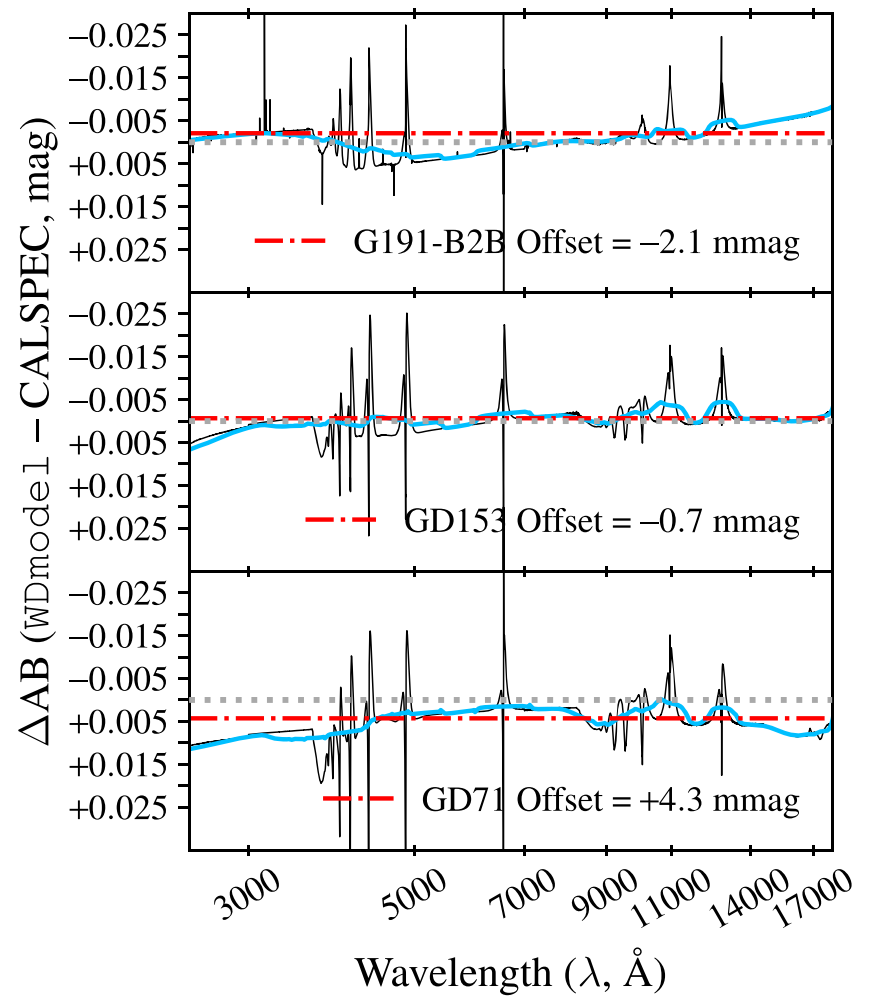

Figure 17. Differences between the CALSPEC SEDs and our inferred SEDs for the three primary standards in $\mathrm{AB}$ magnitudes (black). As the CALSPEC SEDs and our SEDs are computed from different DA WD atmosphere grids, there are detailed differences in the line shapes. In blue, we illustrate the differences smoothed with an order 1 Savitzky-Golay filter having a bandwidth of $1100 \AA$, approximately the width of an optical passband. The median difference (shown in red) between the two SEDs for each star is dominated by the difference in overall normalization arising from the systematic differences in photometry listed in Table 4. The differences in shape of the SED about the median amount to $1-2 \mathrm{mmag}$.

from our inferred SEDs and determine zero-points with these synthetic magnitudes can expect to be on the CALSPEC photometric system to within this amount. This systematic cannot explain the discrepancy in F160W, as a zero-point error would affect all of the apparent magnitudes in a given passband by the same amount.

We note that the CALSPEC system itself may have nonzero $\mathrm{AB}$ offsets. There is no way to quantify the offset between CALSPEC and the true $\mathrm{AB}$ system without using external catalogs, most of which are ground-based and less accurately calibrated, and often also tied to CALSPEC themselves. In Section 9, we discuss a more complex framework to put all the CALSPEC standards and the DA WDs presented in this work on a single photometric system. Such an analysis could incorporate data from laboratory or satellite-borne flux standards to set the absolute $\mathrm{AB}$ zero-points.

\subsection{Identifying the Source of the Residual Bias in F160W}

None of the previously considered sources of systematic error can explain the disagreement between model and observations in F160W seen in Figure 16. To identify the underlying source of the systematic error, we began looking for correlations between the residuals and other quantities derived in our analysis. The residuals between the observations and model are correlated with the apparent magnitude in F160W.
Only one component of our measurement chain can be sensitive to the brightness of the source-the HST/WFC3 detector itself.

We do not see this correlation between residuals and apparent magnitude in any of the WFC3/UVIS passbands. We find that the residuals remain biased if we use the apparent magnitudes determined from instrumental photometry measured with SourceExtractor or DAOPHOT. The F160W exposures are obtained with the MULTIACCUM mode of WFC3, where the signal is sampled multiple times during an exposure. This is used for both cosmic-ray removal and reducing the effective read noise. We found that the measurements we obtained by examining the individual reads were the same as determined from ramp fitting. We manually determined the photometry for a subset of the objects with the largest residuals and found that our measurements were in agreement with the ILAPH measurements.

To exclude any effect arising from an error in the CALPSEC SED of the primary standards, we recomputed zero-points across all passbands, excluding one of G191-B2B, GD 71, and GD 153, and performed the entire analysis with the three resulting sets of apparent magnitudes. The residual bias in F160W persisted across all three sets of results. We note that the F160W residuals of two of the three primary standards (GD 71 and GD 153) are completely consistent with 0 , while the third is only a $2 \sigma$ outlier. This suggests that the origin of the bias is not in the photometry of our program stars, and not the CALSPEC standards. To verify this, we adjusted the settings employed by ILAPH to produce eight different sets of instrumental photometry. The default ILAPH configuration uses a 5 pixel aperture and an annulus from 14 to 21 pixels in F160W. We varied the aperture size from 3 to 9 pixels in steps of 2 pixels, and we used two different annuli, the first from 23 to 46 pixels and the second from 46 to 62 pixels. If the underlying source of the residual bias originates with the instrumental photometry, this test would exhibit a change in the strength of the bias with increasing aperture and annulus size. We found that the bias in F160W increased (i.e., the apparent magnitudes become fainter and more discrepant with model magnitudes) with an increase in both aperture and annulus size above 5 pixels, while the residuals in the UVIS passbands remained consistent with 0 . The dispersion in F160W increases significantly for all aperture sizes at the 3-pixel aperture, as the IR channel of WFC3 has a 0 ". 13 pixel $^{-1}$ scale, and small-aperture photometry is extremely susceptible to centroiding errors. These tests suggest that the discrepancies between model and observations in F160W are due to some unmodeled systematic effect with our WFC3/IR data.

\subsection{Count Rate Nonlinearity}

The correlation of the residuals with apparent F160W magnitude and the sensitivity of the size of the residuals to the number of pixels included in the aperture strongly suggest that the bias is a count rate nonlinearity (CRNL) effect. The calwf3 pipeline corrects for nonlinearity with the total instrumental counts, and we adjust our exposure times to ensure that our measurements have similar $\mathrm{S} / \mathrm{N}$, but no correction is applied based on the count rate of the source. Previous $\mathrm{HgCdTe}$ detectors on HST have suffered from a count-rate-dependent nonlinearity, and the effect has been well characterized for HST/NICMOS (Bohlin et al. 2006; de Jong et al. 2006). The effect in HST/NICMOS amounts to $-0.1 \mathrm{mag}$ $\mathrm{dex}^{-1}$ at $\mathrm{F} 110 \mathrm{~W}$ but is strongly chromatic, decreasing to 
Table 4

Comparison of Observed HST/WFC3 Magnitudes and Synthetic Magnitudes Derived from the CALSPEC SEDs of the Primary Standards

\begin{tabular}{|c|c|c|c|c|c|c|c|c|}
\hline Object & Source & $\mathrm{F} 275 \mathrm{~W}$ & F336W & F475W & $\begin{array}{c}\text { F625W } \\
\text { (AB mag) }\end{array}$ & F775W & F160W & Mean \\
\hline \multirow{3}{*}{ G191-B2B } & Observed & $10.4904(0.0014)$ & $10.8902(0.0014)$ & $11.4988(0.0013)$ & $12.0307(0.0011)$ & $12.4513(0.0013)$ & $13.8853(0.0015)$ & \\
\hline & CALSPEC & 10.4915 & 10.8917 & 11.4995 & 12.0304 & 12.4491 & 13.8851 & \\
\hline & Residual & -0.0011 & -0.0014 & -0.0007 & +0.0003 & +0.0021 & +0.0001 & $0.0000(0.0005)$ \\
\hline \multirow{3}{*}{ GD 153} & Observed & $12.2015(0.0016)$ & $12.5678(0.0015)$ & $13.0998(0.0018)$ & $13.5976(0.0014)$ & $14.0017(0.0014)$ & $15.4139(0.0017)$ & \\
\hline & CALSPEC & 12.2001 & 12.5662 & 13.0979 & 13.5982 & 14.0040 & 15.4141 & \\
\hline & Residual & +0.0015 & +0.0016 & +0.0018 & -0.0006 & -0.0023 & -0.0002 & $+0.0001(0.0006)$ \\
\hline \multirow{3}{*}{ GD 71} & Observed & $11.9888(0.0015)$ & $12.3360(0.0014)$ & $12.7987(0.0015)$ & $13.2789(0.0013)$ & $13.6720(0.0012)$ & $15.0676(0.0019)$ & \\
\hline & CALSPEC & 11.9811 & 12.3271 & 12.7941 & 13.2749 & 13.6720 & 15.0605 & \\
\hline & Residual & +0.0076 & +0.0089 & +0.0046 & +0.0040 & -0.0001 & +0.0071 & $+0.0049(0.0006)$ \\
\hline Zero-point & Mean & $+0.0026(0.0009)$ & $+0.0029(0.0008)$ & $+0.0016(0.0009)$ & $+0.0012(0.0007)$ & $0.0000(0.0007)$ & $+0.0019(0.0010)$ & \\
\hline
\end{tabular}

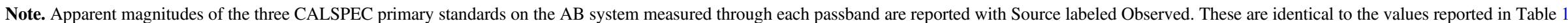

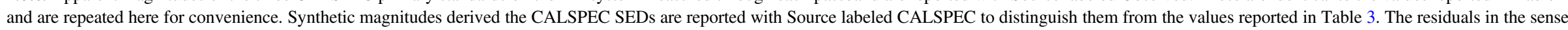

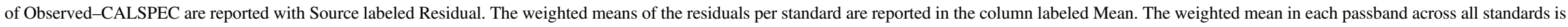
reported with Source labeled Zero-point. Uncertainties on measured or derived quantities are reported parenthetically. All quantities are rounded to $1 / 10$ of a millimagnitude. 


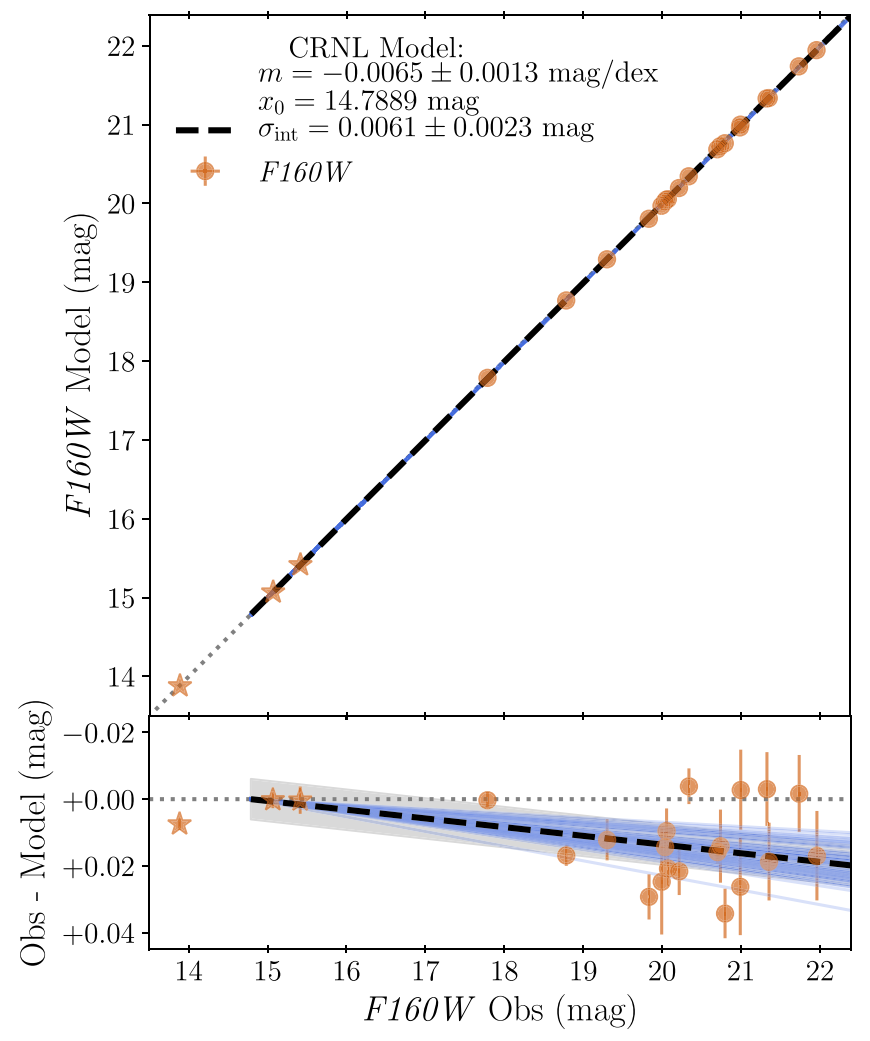

Figure 18. We quantify the CRNL in F160W by fitting the HST/WFC3 UVIS passbands and the spectrum of each object, but excluding F160W. We then compute the synthetic F160W magnitudes from the inferred SED. The model magnitudes are thus completely independent of the observed F160W magnitudes. We model the CRNL as a linear relationship between the observed and synthetic magnitudes (top panel, dashed black line), accounting for the observed and synthetic errors. The CRNL cannot be determined for the three primary standards, as these are used to set the zero-points, and the intercept of the line is fixed to their mean F160W magnitude. The deviation from the 1:1 relationship (dotted gray line) is clearly visible in the residuals (bottom panel). The range of the dispersion about the mean parameters of the linear fit is indicated by the gray shaded region. Random draws from our MCMC are shown in light blue, to illustrate the range of inferred slope.

-0.03 mag dex ${ }^{-1}$ at $\mathrm{F} 160 \mathrm{~W}$, where the negative sign indicates that the observations are fainter than what would be measured in the absence of nonlinearity. The analysis in de Jong (2006) corrects for the nonlinearity using a power law in the count rate, which translates to a linear trend in magnitudes.

We quantify the CRNL for WFC3 IR channel by repeating the analysis as described in Section 6 excluding the F160W observations. The resulting SED parameters are consistent with the values inferred from the full data set, but as F160W was not included in this second analysis, the synthetic F160W model magnitudes and observations are independent. We fit a linear relation between the observed and synthetic magnitudes, accounting for the errors in both quantities, and allowing a dispersion to account for the imperfect fit. While the strength of the CRNL effect and the errors increase with magnitude, unlike a flat dispersion at all magnitudes, we cannot justify a more complex noise model with the limited number of observations available at present. The three CALSPEC standards are used to define the zero-point in F160W, and the CRNL effect can only be measured with respect to these stars. We therefore fix the intercept in our analysis to the mean F160W $=14.7889$ mag of the three primary standards.
The results of our analysis of the CRNL are shown in Figure 18. We find that the slope of the CRNL is $-0.0065 \pm 0.0013 \mathrm{mag} \mathrm{dex}^{-1}$, leading to observed magnitudes that are fainter than would be predicted from the SED model. The lamp of the WFC3 IR channel cannot be operated during observations, preventing a direct measurement where the count rate of sources is enhanced artificially. Previous studies have reported CRNL measurements derived from a comparison of count rates between overlapping passbands imaged with the WFC3 UVIS and IR channels (Riess 2010), and from artificially boosting the count rate of sources with Earth limb shine (Riess \& Petro 2010). Our CRNL measurement is consistent with these previous limits, both of which find that the effect is $<0.01 \mathrm{mag} \mathrm{dex}^{-1}$. It is also consistent with measurements on the WFC3 IR channel flight spares carried out at the Goddard Detector Laboratory presented in Riess (2011). The agreement between these various independent studies leads us to conclude that the residual bias exhibited in F160W is the result of count rate nonlinearity in the WFC3 IR channel.

The complex analysis procedure to transform from our multicycle observations to apparent magnitudes in Section 4 makes it intractable to determine the CRNL directly from the instrumental counts of our images. Our measurement is consistent with previous work but is also more precise than those determinations, so we have elected not to make a correction based on those independent estimates. While the strong chromatic trend of the CRNL with HST/NICMOS leads us to expect a variation with passband, our DA WD program only included observations in F160W. Finally, there is no way to correct the CRNL effect for our data using a measurement from our data. While we expect to be able to account for the CRNL in our analysis of the combined observations from Cycles 20, 22, and 25, we are forced to incorporate the bias into our error budget for this work.

The error in our SEDs of the DA WD stars induced by the CRNL is smaller than the magnitude of the effect in F160W, as the output SEDs are constrained by all of the observed photometry and the spectroscopy and should be robust against a systematic bias in a single passband. Conversely, even though the CRNL effect is only present in F160W, it will have some effect at all wavelengths, as all the data are modeled coherently. We can evaluate the bias in the SEDs caused by the CRNL in F160W by repeating the analysis in Section 6 with F160W excluded entirely. Compared to the results shown in Figure 16, excluding F160W entirely from the analysis reduces the mean residual in $\{\mathrm{F} 275 \mathrm{~W}, \mathrm{~F} 336 \mathrm{~W}, \mathrm{~F} 475 \mathrm{~W}, \mathrm{~F} 625 \mathrm{~W}, \mathrm{~F} 775 \mathrm{~W}\}$ to $\{0$, $0,0+1,-1\}$ mmag. This is a $1-2$ mmag change for each of the WFC3/UVIS passbands. The rms in F625W and F775W is reduced to 2 and $3 \mathrm{mmag}$, respectively, while the standard deviation of the residuals across all passbands is reduced from 6 to 4 mmag. These indicate that the systematic bias caused by our present inability to remove the CRNL is at the $2 \mathrm{mmag}$ level. Because of the CRNL, synthetic WFC3/IR magnitudes derived from our SEDs are likely better predictors of the true flux of our DA WDs than observations with the instrument.

\subsection{The Systematic Error Budget}

The best estimate of the effect on our results from random errors is $6 \mathrm{mmag} \mathrm{rms}$ in any one passband, for any one of our stars. The true effect from random errors is likely closer to $4 \mathrm{mmag}$, as the largest residuals are in F160W, which are 
Table 5

Sources of Systematic Bias and Estimated Effect on the WDmodel SEDs

\begin{tabular}{lc}
\hline \hline Underlying Source of Bias & $\begin{array}{c}\text { Systematic Effect on SED } \\
\text { (mmag) }\end{array}$ \\
\hline \multicolumn{1}{c}{ Effects Caused by Model Misspecification } \\
\hline Model Atmosphere Grid $^{c}<1$ \\
Reddening Model $^{\text {a }}$ & $<1$ \\
Passband Model & $<1$ \\
\hline
\end{tabular}

Effects Caused by Bias in Observations

CALSPEC Flux Scale

4

Count Rate NonLinearity

Note.

${ }^{a}$ An error in the reddening model will cause dispersion rather than bias unless the error is correlated for all our objects. This is extremely unlikely for our allsky network.

systematically biased because of count rate nonlinearity in the WFC3/IR channel. In addition to random effects, we have considered five potential systematic effects in Section 7. We summarize our estimate of their effects in Table 5. We can divide the potential sources of systematic effects we have considered into two categories:

1. Biases that arise because of misspecification of the model.

2. Biases that arise because of miscalibration of the data.

Our analysis is robust against the first of these. We elected to establish DA WDs as standards because the physics that describes their atmospheres is well understood. We chose our objects to be in low-extinction environments and obtained multiband photometry to tightly constrain the reddening. Consequently, any error in our reddening model has negligible impact, and as our standards are spread across the sky, the errors are extremely unlikely to be correlated. While we cannot rule out an error in the response of the $H S T / \mathrm{WFC} 3$, we elected to use reference standards with almost the same colors as our program stars, as this minimizes the effect of our imperfect knowledge of the true system throughput.

We are more sensitive to systematic effects arising with inputs over which we have no control. Any error in the flux scale defined by the three CALSPEC primary standards will propagate to all our SEDs. While the synthetic colors of the SED are set by the model and will remain accurate, the overall fluxes can be systematically off by up to $4 \mathrm{mmag}$, though this is the most conservative estimate of the error possible, and the true error is likely smaller. Additionally, our program has provided the most precise measurement of the count rate nonlinearity in the WFC3/IR detector. While this nonlinearity affects our measurements in F160W only, it has the potential to impact our model at all wavelengths, as this is the reddest passband in our program. Despite this, we found that excluding F160W only causes achromatic 1-2 mmag shifts in the SEDs. Our model is robust against a bias in a single passband precisely because we coherently forward-model all the observations, and their combined statistical weight prevents the biased F160W measurements from torquing the SED significantly.

We note that there is an additional up to $0.5 \%$ error arising from how well the CALSPEC flux scale is tied to the true AB flux scale defined by Oke \& Gunn (1983). This error is achromatic and affects both the CALSPEC primary standards and our DA WD SEDs by the same amount in the same direction, and we therefore do not include it in our systematic error budget. Reducing this error further requires a different experiment and analysis from that described in C19 and this work. We consider such an experiment in Section 9.

\section{Verification and Validation}

In this section, we describe various tests of the model described in Section 6 for internal consistency (verification), as well as against external "truth" (validation).

\subsection{Testing Model Parameters for Objects with Multiple Spectroscopic Observations}

Fifteen of the objects in our sample have more than one spectroscopic observation. Fourteen of these are objects with a spectrum observed in queue mode with Gemini/GMOS. Despite the high $\mathrm{S} / \mathrm{N}$ of the spectra, these observations may suffer from subtle systematic biases that are not ideal for the analysis in this work, as they were executed without the rotator set at the parallactic angle or without a flux standard observed contemporaneously. Moreover, the GMOS spectra are dispersed across three CCDs and stitched together into a single trace. We found sharp discontinuities in the continuum of the reduced spectra, as the gain values were incorrectly set in the pipeline. We re-reduced the spectra for these objects, but the correction for these discontinuities is ad hoc.

While the Gemini/GMOS observations are likely sufficient for most purposes, we elected to obtain at least one MMT/Blue Channel spectrum for 14 objects out of an abundance of caution. As described in Section 3.7, the MMT/Blue Channel spectra generally have lower $\mathrm{S} / \mathrm{N}$ and lower resolution than the Gemini spectra but cover a larger range in wavelength, including the $\mathrm{H} \alpha$ feature, and while they yield slightly weaker constraints on the surface gravity $\log g$, they suffer from fewer systematics. Finally, we observed the primary standard GD 71 multiple times with SOAR across a range of airmass. These 15 objects with at least two spectra each are a valuable test of the consistency of inference with the model described in Section 6.

As described in Section 2.2, the intrinsic DA WD parameters $T_{\text {eff }}$ and $\log g$ are strongly constrained by the spectroscopic observations. $A_{V}$ is also constrained by the spectra. Of the two remaining parameters that determine the SED $\boldsymbol{F}, R_{V}$ is fixed in this work, and $\mu$ is set by our HST/WFC3 photometry. We use the objects with more than one spectrum to verify that the inferred SED parameters are consistent with the parameters listed in Table 2 determined from the spectra with the highest log-likelihood of the observed photometry. The results of this comparison are shown in Figure 19. Despite our spectra being obtained with different instruments, telescopes, sites, and conditions and at different epochs by different observers, the inferred SED parameters are entirely consistent with each other.

\subsection{Correlations between Inferred Extinction and Sodium Absorption Lines in WD Spectra}

The WD spectra used to determine $T_{\text {eff }}$ and $\log g$ provide additional information that can validate our model-fitting process. Specifically, the spectra may show evidence for interstellar extinction through the presence of $\mathrm{Na}$ I D lines. The equivalent width of the sodium absorption features can provide 

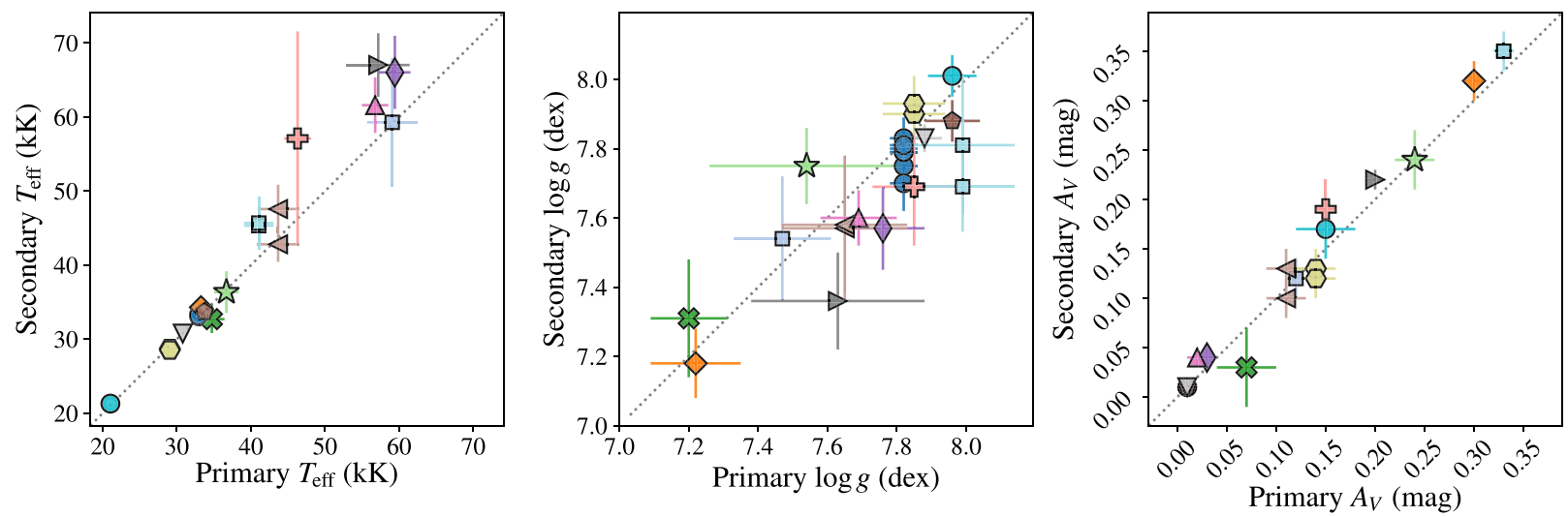

- GD71

SDSS-J010322

$\diamond$ SDSS-J024854

\ SDSS-J081508

SDSS-J102430

ङ SDSS-J111059

$\diamond$ SDSS-J111127

SDSS-J121405

$\triangleleft$ SDSS-J131445

$\triangle$ SDSS-J155745

$\triangleright$ SDSS-J163800

$\nabla$ SDSS-J181424

SDSS-J210150

$\begin{array}{ll}0 & \text { SDSS-J232941 } \\ \square & \text { SDSS-J235144 }\end{array}$

Figure 19. The inferred values of $T_{\text {eff }}$ (left), $\log g$ (middle), and $A_{V}$ (right) for objects (indicated by the marker listed in the legend) with multiple spectra are consistent with each other at the $1.5 \sigma$ level (the gray dotted line indicates a 1:1 relationship). The primary values along the abscissa of both axes are listed in Table 2 and are inferred from our HST/WFC3 photometry and typically the MMT/Blue Channel spectroscopy. These values have the highest log-likelihood of the photometry and typically also have the lowest error on the inferred $T_{\text {eff. }}$ The secondary ordinate values are inferred from the same $H S T$ photometry, together with another spectrum of the same object, typically from Gemini/GMOS. GD 71 has multiple spectra from SOAR. We do not compare the overall normalization parameter $\mu$, as it is determined solely by the HST photometry.

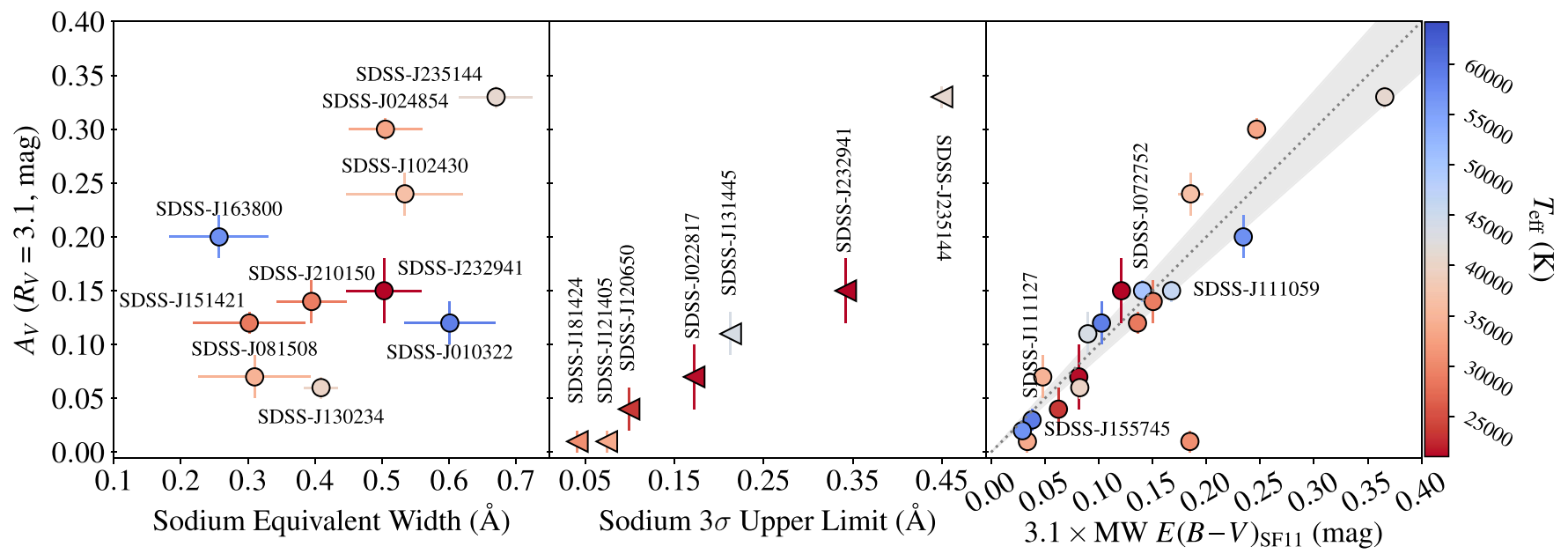

Figure 20. Extinction $A_{V}$ inferred from our model fits compared to independent measurements. Left: $A_{V}$ vs. equivalent width of the Na I D measured from the spectra. The sodium line equivalent widths correlate with $A_{V}(r=0.51)$. Middle: $A_{V}$ vs. $3 \sigma$ upper limits that also correlate with $A_{V}(r=0.96)$. Note that two stars, SDSS J232941 and SDSS J235144, appears in both the left and middle panels, as these objects have two spectra taken at different epochs with different spectrographs at different resolutions. In both cases, one of the spectra had insufficient $\mathrm{S} / \mathrm{N}$ to measure an equivalent width. Right: $A_{V}$ vs. average extinction from the Galactic dust map of Schlafly \& Finkbeiner (2011, denoted as SF11). This average extinction is determined over a region with $5^{\prime}$ radius centered on the position of each DA WD, assuming $R_{V}=3.1$. A 1:1 relationship is indicated by the gray dotted line. Our distant sources suffer extinction but are not behind the full dust column, and our inferred $A_{V}$ will generally be lower than the estimate derived from the dust maps, which is appropriate for extragalactic sources. Schlafly et al. (2016) find the dispersion in $R_{V}$ along different lines of sight to be 0.18 . The $2 \sigma$ region of the extinction $A_{V}$ given the color excess $E(B-V)$ is indicated by the gray shaded region. Points are colored by effective temperature indicated by the color bar at far right.

a reasonably accurate, albeit imprecise, estimate for the extinction along the line of sight (e.g., Poznanski et al. 2012). Given the coarse resolution of our spectra, though, we can expect only a rough correlation between equivalent width and extinction.

For the spectra from the MMT, the $\mathrm{S} / \mathrm{N}$ at the location of the sodium lines is relatively poor as a result of shorter exposure times. The spectra obtained with Gemini have considerably higher $\mathrm{S} / \mathrm{N}$ at the sodium lines, partly as a result of larger aperture and more exposure time, but also because the GMOS spectrograph is typically more sensitive at these wavelengths than the Blue Channel spectrograph. For our analysis, we will consider mainly the Gemini spectra, along with one object from the MMT (SDSS J235144).

We used two techniques to measure the equivalent width after normalizing the shape of the spectrum near the Na I D lines. Both summing the values in the spectra and fitting two Gaussians (with fixed means to match the line separation) yielded essentially identical results. We will use the Gaussian fit equivalent widths. There are 10 objects with detectable lines. For the spectra without obvious lines, we calculated an upper limit for the equivalent width. Based on a prescription from Hobbs (1984), Leonard \& Filippenko (2001) derive this formula for the $3 \sigma$ upper limit of the equivalent width of a feature (in $\AA$ ):

$$
\operatorname{EW}_{\lambda}(3 \sigma)=3 \cdot \Delta \lambda \cdot \Delta I \sqrt{\frac{W_{\text {line }}}{\Delta \lambda \cdot B}} .
$$

Here $\Delta \lambda$ is the width of a resolution element in $\AA, B$ is the width of a resolution element in pixels at the native dispersion, $\Delta I$ is the $1 \sigma$ rms fluctuation of the flux around a normalized continuum level, and $W_{\text {line }}$ is the width of the line feature in $\AA$ 


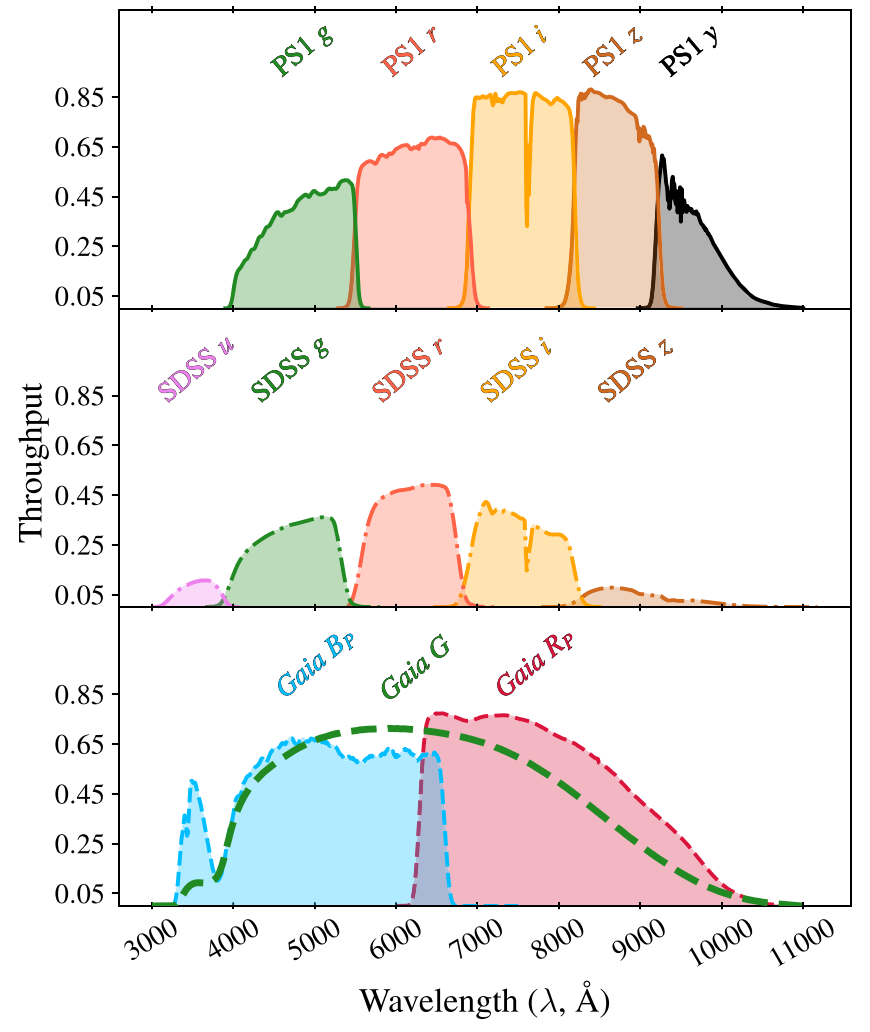

Figure 21. Passband transmissions for surveys/facilities that provide an independent comparison of observed DA WD photometry (from top to bottom: Pan-STARRS PS1, SDSS, and Gaia). The passband names are indicated in the labels above each passband, centered on the pivot wavelength. Throughput curves are illustrated as reported, and we have not normalized them.

(taken to be $10 \AA$ for interstellar $\mathrm{NaID}$ ). $\Delta \lambda$ and $B$ are determined from each spectrum using the FWHM from a line in the comparison lamp near the wavelength of $\mathrm{Na} I \mathrm{D}$ and are likely to be overestimates given the observing conditions. $\Delta I$ is measured from the normalized spectrum. The limit is essentially an estimate of the noise in a resolution element. There are seven spectra with reasonable upper limits.

In Figure 20, we compare the measured sodium equivalent widths and upper limits with the estimates of extinction $A_{V}$ inferred from our model fits to the same spectra. For both measured values and upper limits, the sodium equivalent widths do correlate with the values of $A_{V}$, providing an independent validation that the model-fitting procedure is deriving reasonable estimates of interstellar extinction.

\subsection{Comparing Inferred Extinction to Extinction from Galactic Dust Maps}

We can compare our inferred extinction to each DA WD against an independent estimate of the extinction derived from the Galactic dust maps of Schlafly \& Finkbeiner (2011). Assuming $R_{V}=3.1$, appropriate for diffuse interstellar dust in the Milky Way, we can convert the color excess estimate MW $E(B-V)$ from the dust map to an extinction. This quantity is a measure of the average extinction of extragalactic sources determined from an ensemble of main-sequence stars in a region of radius $5^{\prime}$ centered on the DA WD position. Our inferred $A_{V}$ are line-of-sight estimates from our observations of each DA WD. These two quantities can be substantially different, as is the case for the nearby CALSPEC primary standards, two of which have MW $E(B-V)>0.3 \mathrm{mag}$ despite B14 constraining them to $A_{V}<0.005$ mag. Nevertheless, the extinction inferred for our more distant WDs should be correlated with the integrated extinction determined from the dust map. This is evident in Figure 20, where are our inferred $A_{V}$ are generally smaller than the estimate from the dust map. The dispersion about a 1:1 relationship is consistent with the variation of $R_{V}$ along different lines of sight found by Schlafly et al. (2016).

If $R_{V}$ was significantly different from 3.1 for any specific program star, we would see correlated residuals as a function of wavelength in all our passbands that grow with the estimate of the extinction $A_{V}$. We do not observe any such trend for any object. Moreover, the magnitude of the effect is small for any star, as it is on the order of the difference between the true value of $R_{V}$ and 3.1 times the color excess $E(B-V)$, i.e., $\sim \sigma_{R_{V}} \times E(B-V)=3-4$ mmag for any object. As our stars are spread across the sky, the difference between the latent $R_{V}$ and 3.1 is not correlated across all objects. Any uncertainty that arises from the difference between the true reddening law and the canonical F99 model is a random effect, i.e., fixing $R_{V}$ to 3.1 may add dispersion but does not cause a systematic bias.

\subsection{Expected Magnitudes on Common Photometric Systems}

In this section, we compare our synthetic photometry to independently observed photometry from PS1, SDSS, and Gaia. While comparing the synthetic photometry of our DA WDs against catalogs from other surveys can be informative, it is also challenging with ground-based surveys owing to the systematic effects listed in Section 2.1. Indeed, avoiding these systematic effects was the motivation for us to obtain aboveatmosphere HST photometry for our program. Nevertheless, these data are a valuable test of consistency. Historically, optical surveys have used such comparisons of synthetic and observed photometry from spectrophotometric references such as $\mathrm{BD}+17^{\circ} 4708$ to quantify the inconsistency of the survey flux scale with the $\mathrm{AB}$ flux scale and derive offsets to their natural system magnitudes. We account for these offsets where they are available in the literature. For surveys that do not report calibrated $\mathrm{AB}$ magnitudes, we compare synthetic and observed natural system photometry up to an overall constant. The passband responses of the different surveys are shown in Figure 21, and machine-readable tables are included within our WDmodel package, together with routines to generate synthetic photometry from our SED models. The results of the comparison of each survey are discussed below.

\subsubsection{Comparison with PS1 DR1}

Scolnic et al. (2015, hereafter S15) have derived offsets between photometry from the first $1.5 \mathrm{yr}$ of PS1 (Schlafly et al. 2012) and the true $A B$ flux scale using comparisons of the synthetic photometry of seven CALSPEC standards. ${ }^{32}$ This initial release of PS1 photometry has been superseded by the PanSTARRS PS1 Data Release ${ }^{33}$ (DR1) photometry. The internal PS1 calibration ladder is described in Magnier et al. (2013), and

\footnotetext{
32 The S15 analysis used the stisnic_005 SEDs of blue CALSPEC standards Snap-1, WD1657+343, and LDS749B, and red CALSPEC standards SF1615+001A, Snap-2, and C26202, as well as the stisnic_004 SED of red standard KF06T2. GD153 is included with the other standards to determine the $\mathrm{AB}$ offsets for $z_{\mathrm{PS} 1}$ and $y_{\mathrm{PS} 1}$, as the gri $_{\mathrm{PS} 1}$ measurements are saturated.

33 https://panstarrs.stsci.edu/
} 
Table 6

Comparison of PS1 DR1 Aperture Magnitudes and Synthetic Magnitudes Derived from Our DA White Dwarf SEDs

\begin{tabular}{|c|c|c|c|c|c|c|c|c|c|}
\hline \multirow{2}{*}{ Object } & \multirow{2}{*}{ PS1 ObjID } & \multicolumn{2}{|c|}{ PS1 $g$} & \multicolumn{2}{|c|}{ PS1 $r$} & \multicolumn{2}{|c|}{ PS1 $i$} & \multicolumn{2}{|c|}{ PS $1 z$} \\
\hline & & \multicolumn{6}{|c|}{ mag (mmag) } & & \\
\hline Snap-1 & 171512473989538506 & $15.506(6)$ & 15.498 & $15.892(4)$ & 15.894 & $16.207(2)$ & 16.202 & $16.425(2)$ & 16.425 \\
\hline WD $1657+343$ & 149172547130238286 & $16.230(4)$ & 16.228 & $16.700(2)$ & 16.693 & $17.074(2)$ & 17.074 & $17.375(7)$ & 17.360 \\
\hline SF $1615+001 \mathrm{~A}$ & 108002445593133309 & $16.988(4)$ & 16.991 & $16.560(2)$ & 16.563 & $16.381(2)$ & 16.384 & $16.314(3)$ & 16.317 \\
\hline Snap-2 & 174682449420946620 & $16.443(5)$ & 16.443 & $16.053(3)$ & 16.045 & $15.912(3)$ & 15.905 & $15.873(2)$ & 15.874 \\
\hline $\mathrm{C} 2602$ & 74560531369524156 & $16.669(4)$ & 16.673 & $16.365(5)$ & 16.368 & $16.258(4)$ & 16.264 & $16.250(3)$ & 16.243 \\
\hline KF 06T2 & 188132696582938100 & $14.406(2)$ & 14.418 & $13.613(4)$ & 13.607 & $13.272(\mathrm{NaN})$ & 13.260 & $13.093(1)$ & 13.087 \\
\hline GD 153 & 134431942595767273 & $13.134(7)$ & 13.128 & $13.598(1)$ & 13.591 & $13.990(11)$ & 13.978 & $14.261(2)$ & 14.263 \\
\hline CALSPEC $\mu, \sigma$ & & $-0.006(1)$ & 0.006 & $+0.003(1)$ & 0.005 & $+0.001(1)$ & 0.005 & $+0.003(1)$ & 0.006 \\
\hline SDSS J010322 & 107580158424764461 & $19.093(10)$ & $19.120(5)$ & $19.570(19)$ & $19.563(5)$ & $19.979(17)$ & $19.933(5)$ & $20.130(64)$ & $20.209(7)$ \\
\hline SDSS J022817 & 97850370715284759 & $19.837(14)$ & $19.827(11)$ & $20.188(53)$ & $20.163(6)$ & $20.523(36)$ & $20.477(7)$ & $20.803(117)$ & $20.728(10)$ \\
\hline SDSS J024854 & 148510422289556735 & $18.351(7)$ & $18.392(8)$ & $18.699(6)$ & $18.740(5)$ & $18.972(12)$ & $19.052(3)$ & $19.198(31)$ & $19.296(5)$ \\
\hline SDSS J072752 & 146681119698436001 & $18.018(10)$ & $18.026(3)$ & $18.475(11)$ & $18.450(2)$ & $18.806(12)$ & $18.809(2)$ & $19.127(24)$ & $19.079(3)$ \\
\hline SDSS J081508 & 117031237865415713 & $19.781(40)$ & $19.747(5)$ & $20.328(37)$ & $20.180(6)$ & $20.625(73)$ & $20.547(7)$ & $20.710(165)$ & $20.823(7)$ \\
\hline SDSS J102430 & 107351561288148089 & $18.885(9)$ & $18.936(9)$ & $19.292(23)$ & $19.311(7)$ & $19.440(98)$ & $19.641(12)$ & $19.758(31)$ & $19.896(18)$ \\
\hline SDSS J111059 & 87401677476402284 & $17.895(5)$ & $17.889(2)$ & $18.302(9)$ & $18.307(2)$ & $18.607(15)$ & $18.664(2)$ & $18.957(26)$ & $18.934(3)$ \\
\hline SDSS J111127 & 155931678637970024 & $18.412(15)$ & $18.456(3)$ & $18.886(11)$ & $18.929(3)$ & $19.260(11)$ & $19.319(3)$ & $19.586(16)$ & $19.607(3)$ \\
\hline SDSS J120650 & 110431817100904541 & $18.693(10)$ & $18.691(4)$ & $19.096(29)$ & $19.056(5)$ & $19.388(24)$ & $19.388(6)$ & $19.645(34)$ & $19.648(7)$ \\
\hline SDSS J121405 & 162761835213366810 & $17.779(5)$ & $17.787(4)$ & $18.236(7)$ & $18.229(3)$ & $18.570(10)$ & $18.605(2)$ & $18.849(17)$ & $18.890(3)$ \\
\hline SDSS J130234 & 120251956434903476 & $17.052(3)$ & $17.066(3)$ & $17.494(3)$ & $17.505(2)$ & $17.858(6)$ & $17.877(2)$ & $18.114(9)$ & $18.157(3)$ \\
\hline SDSS J131445 & 104111986877165205 & $19.078(14)$ & $19.130(7)$ & $19.556(21)$ & $19.562(6)$ & $19.887(40)$ & $19.928(4)$ & $20.240(69)$ & $20.203(6)$ \\
\hline SDSS J151421 & 108952285886717975 & $15.720(2)$ & $15.729(4)$ & $16.101(4)$ & $16.110(2)$ & $16.434(2)$ & $16.448(2)$ & $16.715(5)$ & $16.712(2)$ \\
\hline SDSS J155745 & 174922394391413855 & $17.487(5)$ & $17.505(2)$ & $17.958(7)$ & $17.978(2)$ & $18.356(5)$ & $18.367(2)$ & $18.647(11)$ & $18.656(3)$ \\
\hline SDSS J163800 & 108942495015106395 & $18.860(13)$ & $18.864(6)$ & $19.314(22)$ & $19.277(4)$ & $19.611(13)$ & $19.627(4)$ & $19.816(53)$ & $19.891(5)$ \\
\hline SDSS J181424 & 202682736002931825 & $16.573(5)$ & $16.568(2)$ & $17.007(3)$ & $16.999(1)$ & $17.358(4)$ & $17.369(2)$ & $17.651(9)$ & $17.650(2)$ \\
\hline SDSS J210150 & 101083154611083390 & $18.652(9)$ & $18.677(6)$ & $19.052(8)$ & $19.056(3)$ & $19.410(18)$ & $19.393(4)$ & $19.703(33)$ & $19.655(6)$ \\
\hline SDSS J232941 & 108223524222323007 & $18.134(6)$ & $18.163(6)$ & $18.452(5)$ & $18.468(5)$ & $18.772(8)$ & $18.762(4)$ & $19.003(17)$ & $19.000(5)$ \\
\hline SDSS J235144 & 153513579345744806 & $18.085(4)$ & $18.099(6)$ & $18.447(13)$ & $18.450(4)$ & $18.776(10)$ & $18.764(3)$ & $19.100(36)$ & $19.010(4)$ \\
\hline DA WD $\mu, \sigma$ & & $-0.012(2)$ & 0.019 & $-0.004(2)$ & 0.017 & $-0.013(2)$ & 0.021 & $-0.007(4)$ & 0.030 \\
\hline
\end{tabular}

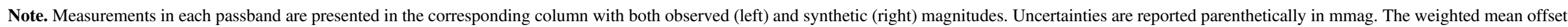

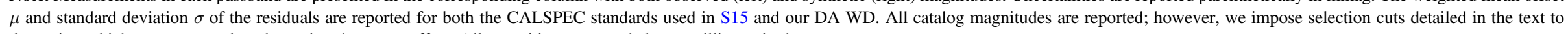
determine which stars are used to determine the mean offset. All quantities are rounded to a millimagnitude. 


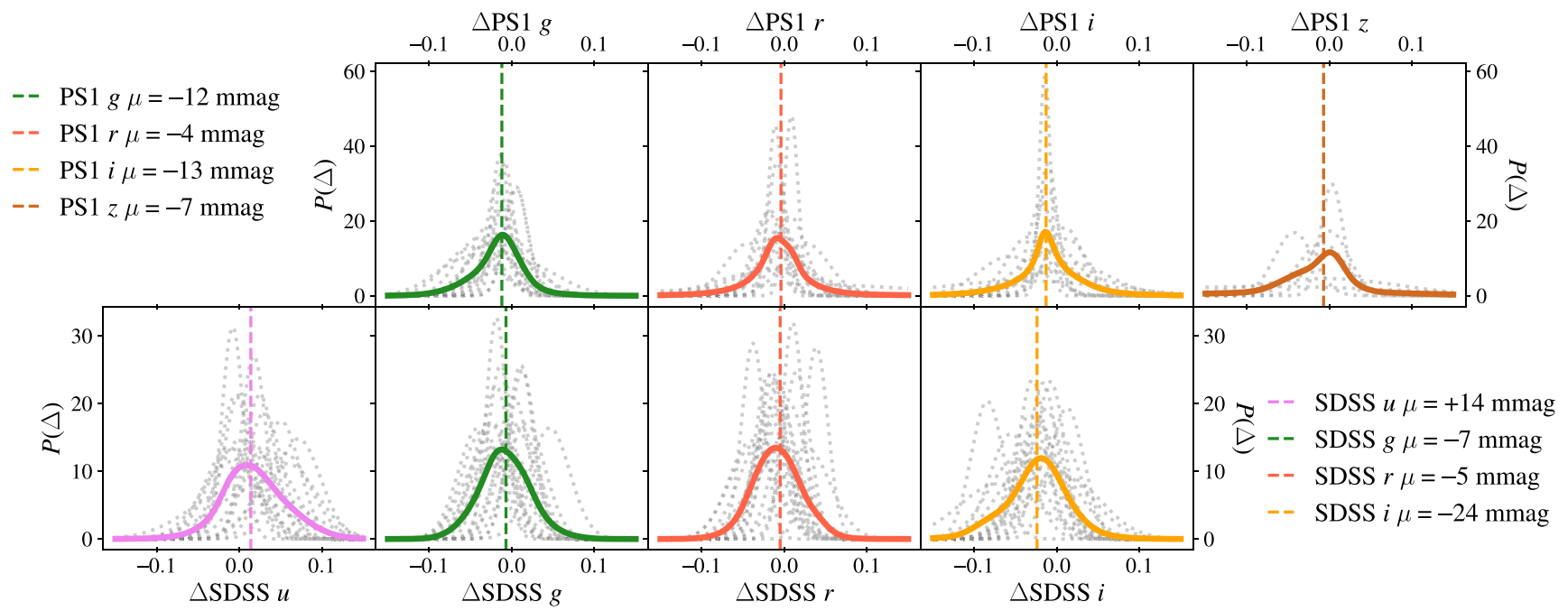

Figure 22. Distribution of residuals of our DA WDs in PS1 (top row) and SDSS (bottom) row. Residuals and associated uncertainties for each star are indicated by the gray dotted Gaussian distributions. A kernel density estimate of the distribution of the residuals is indicated by the solid colored line in each panel. The dashed colored line indicates the weighted mean offset from zero in each band. The values of these offsets are presented in Tables 6 and 7, as well as the legend for each row. The reported uncertainties are scaled by a factor of 2.5 and 1.2 for PS1 and SDSS, respectively, to obtain a reduced $\chi^{2}$ statistic of unity.

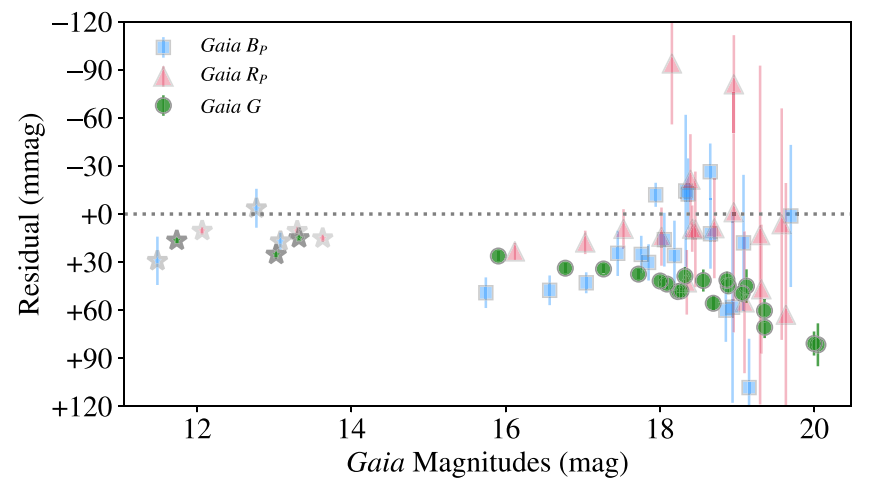

Figure 23. Residuals of CALSPEC primary standards (stars) and our DA WDs (markers indicated in the legend for each passband) vs. magnitude. The Gaia $G$ residuals exhibit bias and a pronounced nonlinearity with magnitude, not seen with other surveys. Casagrande \& VandenBerg (2018) also report a trend with respect to CALSPEC for $6 \mathrm{mag} \leqslant G \leqslant 16.5 \mathrm{mag}$.

the DR1 calibration is described in Magnier et al. (2016), which applies the results of the S15 analysis. There have been numerous changes in the PS1 Image Processing Pipeline between the catalogs used in S15 and the DR1, and we therefore recompute the $\mathrm{AB}$ offsets using the observed DR1 photometry and the same CALSPEC standards used in the S15 analysis.

We use aperture photometry from the PS1 DR1 "MeanObject" table, which we test against the bitmask $0 \times 1 \mathrm{C} 138$ - see Flewelling et al. (2016) for a detailed description of the PS1 DR1 schema, and Table 15 for an explanation of the meaning of each bit. We also require that the reported photometry be the average of at least five detections in each passband and have $\mathrm{S} / \mathrm{N} \geqslant 10$. Finally, we impose a restriction that the PS1 DR1 and aperture magnitudes must agree to within 0.05 mag to exclude contaminated sources. All DR1 photometries of CALSPEC standard LDS749B are removed because of these selection criteria, and we exclude this standard from the comparison. KF06T2 has a valid $i_{\mathrm{PS} 1}$ magnitude, but the uncertainty is reported as NaN. We report this measurement as presented in the PS1 DR1 catalogs but do not include it in determining the mean $\mathrm{AB}$ offset.
Additionally, while we can determine an $\mathrm{AB}$ offset in $y_{\mathrm{PS} 1}$ from the bright CALSPEC standards, there is no DR1 photometry of our DA WDs that matches our selection criteria.

The result of our analysis to determine the $\mathrm{AB}$ offsets from the CALSPEC standard used in S15 and our DA WDs is presented in Table 6. These indicate that DR1 is consistent with the analysis in S15 to within a few millimagnitudes in griz $_{\mathrm{PS} 1}$. These offsets remain consistent when considering the median difference or the $3 \sigma$-clipped difference.

We find the following weighted mean offsets between the PS1 DR1 magnitudes and the synthetic magnitudes of our DA WDs in the PS1 passbands: $\{g, r, i, z\}_{\mathrm{PS} 1}=\{-12,-4,-13$, $-7\}$ mmag. We propagate the uncertainties on observed and synthetic magnitudes to uncertainties in the weighted mean, which are 2-4 mmag. The sign and scale of the AB offsets are consistent across passbands, indicating a real, albeit small, difference between the PS1 and CALSPEC flux scale.

The offsets in $g r_{\mathrm{PS} 1}$ determined from our DA WDs are comparable to the offsets determined from the CALSPEC standards. However, the reported uncertainties on the observations of our DA WDs do not describe the dispersion of the residuals. The reduced $\chi^{2}$ statistic for our faint DA WDs is much higher than for the bright CALSPEC standards. This is likely why the offsets in $i z_{\mathrm{PS} 1}$ are larger than those determined directly from the bright CALSPEC standards, which are additionally much redder on the mean than our DA WDs. We find that the PS1 errors must be scaled by a factor of 2.5 for a reduced $\chi^{2}$ of unity. The distribution of residuals for PS1 and SDSS (discussed in the following subsection) is shown in Figure 22.

We obtained additional imaging of our DA WDs from the Foundation survey, operated on the PS1 telescope, but reduced with the photpipe image processing pipeline (originally described in Rest et al. 2005, but significantly updated thereafter). The Foundation PS1 images indicate that the photometric repeatability is $\sim 1.5 \%$. The $\mathrm{AB}$ offsets determined from the Foundation PS1 images are correlated with those determined from our comparison to the PS1 DR1 catalog, as the latter is used to determine the zero-points of the former. We will use these data to constrain the temporal variability of our 
Table 7

Comparison of SDSS DR12 PSF Magnitudes and Synthetic Magnitudes Derived from Our DA White Dwarf SEDs

\begin{tabular}{|c|c|c|c|c|c|c|c|c|c|}
\hline \multirow{2}{*}{ Object } & \multirow{2}{*}{ SDSS ID } & \multicolumn{2}{|c|}{ SDSS $u$} & \multicolumn{2}{|c|}{ SDSS $g$} & \multicolumn{2}{|c|}{ SDSS $r$} & \multicolumn{2}{|c|}{ SDSS $i$} \\
\hline & & \multicolumn{6}{|c|}{$\overline{m a g}(\mathrm{mmag})$} & & \\
\hline SDSS J010322 & SDSS J010322.19-002047.7 & $18.626(45)$ & $18.631(5)$ & $19.056(28)$ & $19.067(6)$ & $19.546(21)$ & $19.560(5)$ & $19.912(29)$ & $19.921(5)$ \\
\hline SDSS J022817 & SDSS J022817.16-082716.4 & $19.798(42)$ & $19.775(11)$ & $19.785(24)$ & $19.806(11)$ & $20.153(29)$ & $20.163(6)$ & $20.398(40)$ & $20.467(7)$ \\
\hline SDSS J024854 & SDSS J024854.96+334548.2 & $18.116(22)$ & $18.115(7)$ & $18.361(18)$ & $18.357(8)$ & $18.721(17)$ & $18.738(5)$ & $18.957(16)$ & $19.042(3)$ \\
\hline SDSS J072752 & SDSS J072752.76+321416.0 & $17.569(15)$ & $17.570(2)$ & $17.957(10)$ & $17.976(3)$ & $18.457(10)$ & $18.447(2)$ & $18.768(14)$ & $18.797(2)$ \\
\hline SDSS J081508 & SDSS J081508.78+073145.8 & $19.416(29)$ & $19.358(8)$ & $19.676(19)$ & $19.700(5)$ & $20.203(24)$ & $20.177(6)$ & $20.538(33)$ & $20.535(7)$ \\
\hline SDSS J102430 & SDSS J102430.93-003207.0 & $18.577(28)$ & $18.588(9)$ & $18.889(22)$ & $18.896(10)$ & $19.305(22)$ & $19.309(7)$ & $19.596(28)$ & $19.631(12)$ \\
\hline SDSS J111059 & SDSS J111059.42-170954.2 & 17.477 (17) & $17.447(4)$ & $17.858(19)$ & $17.841(3)$ & $18.312(17)$ & $18.305(2)$ & $18.620(18)$ & $18.653(2)$ \\
\hline SDSS J111127 & SDSS J111127.30+395628.0 & $17.984(26)$ & $17.930(4)$ & $18.407(19)$ & $18.398(3)$ & $18.918(14)$ & $18.926(3)$ & $19.282(19)$ & $19.307(3)$ \\
\hline SDSS J120650 & SDSS J120650.40+020142.4 & $18.541(24)$ & $18.553(4)$ & $18.650(23)$ & $18.663(4)$ & $19.028(22)$ & $19.055(5)$ & $19.328(27)$ & $19.377(6)$ \\
\hline SDSS J121405 & SDSS J121405.11+453818.5 & $17.370(25)$ & $17.378(3)$ & $17.711(24)$ & $17.740(4)$ & $18.211(19)$ & $18.227(3)$ & $18.538(21)$ & $18.593(2)$ \\
\hline SDSS J130234 & SDSS J130234.43+101238.9 & $16.614(16)$ & $16.619(2)$ & $16.976(20)$ & $17.016(3)$ & $17.463(23)$ & $17.503(2)$ & $17.840(24)$ & $17.865(2)$ \\
\hline SDSS J131445 & SDSS J131445.05-031415.5 & $18.716(26)$ & $18.683(5)$ & $19.045(20)$ & $19.080(7)$ & $19.522(23)$ & $19.560(6)$ & $19.927(36)$ & $19.917(4)$ \\
\hline SDSS J151421 & SDSS J151421.27+004752.8 & $15.482(12)$ & $15.464(2)$ & $15.681(12)$ & $15.694(4)$ & $16.089(14)$ & $16.108(2)$ & $16.421(14)$ & $16.438(2)$ \\
\hline SDSS J155745 & SDSS J155745.39+554609.7 & $16.975(10)$ & $16.983(2)$ & $17.493(20)$ & $17.447(2)$ & $17.990(13)$ & $17.975(2)$ & $18.334(19)$ & $18.355(2)$ \\
\hline SDSS J163800 & SDSS J163800.36+004717.7 & $18.461(18)$ & $18.410(5)$ & $18.826(11)$ & $18.815(6)$ & $19.269(16)$ & $19.274(4)$ & $19.597(25)$ & $19.616(4)$ \\
\hline SDSS J181424 & SDSS J181424.12+785402.9 & $16.236(15)$ & $16.212(2)$ & $16.498(19)$ & $16.524(2)$ & $16.959(11)$ & $16.997(2)$ & $17.344(17)$ & $17.357(2)$ \\
\hline SDSS J210150 & SDSS J210150.65-054550.9 & $18.483(21)$ & $18.412(9)$ & $18.655(12)$ & $18.643(6)$ & $19.042(14)$ & $19.055(3)$ & $19.385(21)$ & $19.382(4)$ \\
\hline SDSS J232941 & SDSS J232941.32+001107.8 & $18.173(25)$ & $18.156(3)$ & $18.149(14)$ & $18.147(7)$ & $18.453(12)$ & $18.468(5)$ & $18.745(14)$ & $18.752(4)$ \\
\hline SDSS J235144 & SDSS J235144.29+375542.6 & $17.760(23)$ & $17.747(4)$ & $18.042(13)$ & $18.061(6)$ & $18.487(11)$ & $18.448(4)$ & $18.766(17)$ & $18.754(3)$ \\
\hline DA WD $\mu, \sigma$ & & $+0.014(6)$ & 0.025 & $-0.007(4)$ & 0.021 & $-0.005(4)$ & 0.020 & $-0.024(5)$ & 0.025 \\
\hline
\end{tabular}

Note. The format of this table matches Table 6 . 
Table 8

Comparison of Gaia DR2 Magnitudes and Synthetic Magnitudes Derived from Our DA White Dwarf SEDs

\begin{tabular}{|c|c|c|c|c|c|c|c|}
\hline \multirow{2}{*}{$\begin{array}{l}\text { Object } \\
\text { G191-B2B }\end{array}$} & Gaia DR2 ID & \multicolumn{2}{|c|}{ Gaia $B_{P}$} & \multicolumn{2}{|c|}{$\frac{\text { Gaia } R_{P}}{\text { mag (mmag) }}$} & \multicolumn{2}{|c|}{ Gaia $G$} \\
\hline & 266077145295627520 & $11.487(15)$ & $11.458(2)$ & $12.067(2)$ & $12.057(1)$ & $11.738(1)$ & $11.722(2)$ \\
\hline GD 153 & 3944400490365194368 & $13.081(5)$ & $13.064(2)$ & $13.629(1)$ & $13.614(2)$ & $13.322(1)$ & $13.307(2)$ \\
\hline GD 71 & 3348071631670500736 & $12.770(12)$ & $12.774(2)$ & $13.299(2)$ & $13.289(2)$ & $13.026(2)$ & $13.001(2)$ \\
\hline CALSPEC $\mu, \sigma$ & & $+0.015(5)$ & 0.030 & $+0.012(1)$ & 0.037 & +0.018 & 0.017 \\
\hline SDSS J010322 & 2536159496590552704 & $19.154(30)$ & $19.046(5)$ & $19.577(72)$ & $19.571(6)$ & $19.356(4)$ & $19.285(5)$ \\
\hline SDSS J022817 & 5176546064064586624 & 19.869 (139) & $19.823(10)$ & $20.192(141)$ & $20.130(8)$ & $20.046(10)$ & $19.964(9)$ \\
\hline SDSS J024854 & 139724391470489472 & $18.333(47)$ & $18.347(7)$ & $18.704(31)$ & $18.695(4)$ & $18.561(3)$ & $18.520(6)$ \\
\hline SDSS J072752 & 892231562565363072 & $17.944(7)$ & $17.956(3)$ & $18.458(36)$ & $18.448(3)$ & $18.232(3)$ & $18.184(3)$ \\
\hline SDSS J081508 & 3097940536009636992 & $19.695(44)$ & $19.694(6)$ & $20.278(166)$ & $20.187(8)$ & $19.996(5)$ & $19.915(6)$ \\
\hline SDSS J102430 & 3830980604624181376 & $18.940(59)$ & $18.882(8)$ & $19.297(105)$ & $19.284(12)$ & $19.120(5)$ & $19.075(9)$ \\
\hline SDSS J111059 & 3559181712491390208 & $17.852(11)$ & $17.822(3)$ & $18.347(20)$ & $18.304(2)$ & $18.089(2)$ & $18.045(2)$ \\
\hline SDSS J111127 & 765355922242992000 & $18.365(22)$ & $18.378(3)$ & $18.955(75)$ & $18.956(3)$ & $18.690(3)$ & $18.634(3)$ \\
\hline SDSS J120650 & 3891742709551744640 & $18.651(17)$ & $18.677(5)$ & $18.957(30)$ & $19.038(6)$ & $18.885(2)$ & $18.840(5)$ \\
\hline SDSS J121405 & 1539041748872771968 & $17.757(11)$ & $17.732(4)$ & $18.154(38)$ & $18.248(2)$ & $18.002(1)$ & $17.960(3)$ \\
\hline SDSS J130234 & 3734528631432609920 & $17.044(6)$ & $17.001(3)$ & $17.527(12)$ & $17.518(2)$ & $17.268(1)$ & $17.234(3)$ \\
\hline SDSS J131445 & 3684543213630134784 & $19.082(42)$ & $19.064(6)$ & $19.631(82)$ & $19.568(5)$ & $19.354(4)$ & $19.294(6)$ \\
\hline SDSS J151421 & 4419865155422033280 & $15.743(9)$ & $15.694(3)$ & $16.119(5)$ & $16.096(2)$ & $15.905(1)$ & $15.879(3)$ \\
\hline SDSS J155745 & 1621657158502507520 & $17.452(14)$ & $17.427(2)$ & $18.019(18)$ & $18.005(2)$ & $17.721(2)$ & $17.683(2)$ \\
\hline SDSS J163800 & 4383979187540364288 & $18.853(19)$ & $18.793(5)$ & $19.313(40)$ & $19.266(5)$ & $19.065(2)$ & $19.015(5)$ \\
\hline SDSS J181424 & 2293913930823813888 & $16.570(9)$ & $16.522(2)$ & $17.031(7)$ & $17.013(2)$ & $16.773(2)$ & $16.739(2)$ \\
\hline SDSS J210150 & 6910475935427725824 & $18.654(21)$ & $18.642(6)$ & $19.095(44)$ & $19.040(5)$ & $18.867(2)$ & $18.826(5)$ \\
\hline SDSS J232941 & 2644572064644349952 & $18.187(21)$ & $18.161(6)$ & $18.394(28)$ & $18.416(4)$ & $18.323(2)$ & $18.284(5)$ \\
\hline SDSS J235144 & 2881271732415859072 & $18.056(16)$ & $18.040(5)$ & $18.417(14)$ & $18.408(4)$ & $18.272(2)$ & $18.224(5)$ \\
\hline DA WD $\mu, \sigma$ & & $+0.027(3)$ & 0.032 & $+0.017(4)$ & 0.040 & $+0.041(1)$ & 0.015 \\
\hline
\end{tabular}

Note. The format of this table matches Table 6.

standards together with our LCO observations and establish secondary standards around our DA WDs in future work.

\subsubsection{Comparison with SDSS DR12}

Betoule et al. (2013) derive AB offsets for SDSS using careful measurements of the three CALSPEC primary standards with the photometric telescope transferred to the primary survey telescope. Unfortunately, these determinations of the magnitudes of the primary standards differ significantly from the reported SDSS DR12 (Alam et al. 2015) photometry, which is saturated in most passbands. As we cannot independently recompute the AB offsets for DR12, we compare our synthetic magnitudes directly to the reported SDSS DR12 PSF magnitudes in Table 7.

The uncertainties on the SDSS DR12 PSF magnitudes accurately describe the dispersion in $u g r$. There is much larger dispersion in SDSS $i z$, where our DA WDs are the most faint. Several of our targets do not have reliable photometry in the $z$ band, and we exclude it from this comparison. While we impose the same $S / N \geqslant 10$ threshold we used for the PS1 comparison, all reported SDSS DR12 photometries satisfy this criterion. We find the following weighted mean offsets between the SDSS DR12 magnitudes and the synthetic magnitudes of our standards in the SDSS passbands: $\{u, g, r, i\}_{\mathrm{PS} 1}=\{+14$, $-7,-5,-24\}$ mmag. The reported uncertainties from SDSS are reasonable, with the reduced $\chi^{2}$ statistic indicating that they must be scaled by only a factor of 1.2 to fully describe the dispersion. The standard deviation of the SDSS DR12 residuals is larger than we find for PS1, indicating that the latter does have more internally consistent photometry, albeit with significantly underestimated uncertainties.

The offset in $g r$ is consistent with zero, and the offset in $u$ is only significant at the $2 \sigma$ level. The residuals in the $i$ band are not significant at the $1 \sigma-2 \sigma$ level for any individual object but are consistent across all of our 19 DA WDs. This likely reflects a real difference between the CALSPEC flux scale and the SDSS DR12 flux scale. The -24 mmag AB offset in $i$ agrees with the corresponding value of $-27 \mathrm{mmag}$ determined by Betoule et al. (2013).

\subsubsection{Comparison with Gaia DR2}

Gaia provides photometry and parallax measurements (Evans et al. 2018; Riello et al. 2018) for most of our stars (these parallax measurements are reported in Table 1 of C19). For our faint DA WDs, the Gaia parallax errors have a mean precision of $25 \%$. Additionally, the parallax errors increase as a function of magnitude, with three sources having relative errors of $>50 \%$. Two sources in our sample do not have any reported parallax. In contrast to the heterogeneous parallax measurements, we increased exposure times for our spectroscopic and $H S T$ observations to ensure that all our DA WDs have comparable $\mathrm{S} / \mathrm{N}$.

Despite their heterogeneity, there are potential gains to incorporating parallax measurements into our inference. Intrinsic DA WD parameters derived from Gaia observations with $\mathrm{S} / \mathrm{N}>20$ have been compared against measurements inferred from Pan-STARRS and SDSS (Gentile Fusillo et al. 2019), and these are in good agreement. The Gaia parallax measurements can be very useful for distinguishing double-degenerate systems 
that masquerade as a single star. However, there are potential systematic issues with modeling the Gaia measurements together with our spectroscopic and photometric observations. Tremblay et al. (2019) compare DA WDs with intrinsic parameters that are well measured from spectroscopy to stellar parameters derived from Gaia measurements and find that photometric and spectroscopic temperature scales differ systematically by a few percent. Additionally, there is a residual systematic in the inferred values of $\log g$ for DA WDs with $11,000 \mathrm{~K}<T_{\text {eff }}<13,000 \mathrm{~K}$. As Gaia parallaxes are not currently available for all our stars and incorporating the extant measurements into the likelihood function in Section 6 could potentially introduce systematics, we do not use them directly at this time.

Comparison with the Gaia measurements is still informative, and the mission also reports photometry. These photometric observations are reported with respect to Vega by default, but the data release also provides AB-based zero-points. For consistency with the remainder of this work, we use these AB zero-points. We measure the offsets by comparing the Gaia DR2 photometry with synthetic photometry of our SEDs of the CALSPEC primary standards. We then determined the offsets between the DR2 and synthetic photometry of our DA WDs. The results of the comparison are presented in Table 8. As with the comparison against SDSS, we require that the $\mathrm{S} / \mathrm{N}$ be $\geqslant 10$. This rejects one measurement in $B_{P}$ and two in $R_{P}$. These rejected measurements are consistent with our synthetic magnitudes to within $1 \sigma$ of the reported photometric uncertainties.

We find offsets of $\left\{B_{P}, R_{P}, G\right\}=\{+27,+17,+41\}$ mmag from our DA WDs. These offsets are consistent with those determined from the CALSPEC primary standards, where we find offsets of $\{+15,+13,+18\}$ mmag. The residuals in Gaia $G$ show an increasing trend with faint amplitudes (Figure 23). Weak trends can be seen in the other passbands but are less significant because of the much larger uncertainties near the faint limit. Casagrande \& VandenBerg (2018) report on this bias using an independent comparison to CALSPEC and provide a linear correction valid from $6 \mathrm{mag} \leqslant G \leqslant 16.5 \mathrm{mag}$. Their linear correction removes the linear component of the residual, but a nonlinear component remains. While it is possible to define an empirical relation and model the nonlinearity, without understanding the physical origin of this effect it cannot be properly mitigated. No similar trends are seen in the comparisons with PS1 and SDSS. The reported uncertainties on the observations of our DA WDs do not describe the dispersion of the residuals. The reduced $\chi^{2}$ statistic is much greater than unity for all the Gaia passbands, indicating that the uncertainties are underestimated, or that there are additional sources of dispersion with the photometers that have not been accounted for.

\section{Summary and Discussion}

In N16, we identified various potential improvements of our proof-of-concept analysis. This work implements every one of those improvements, as well as refining other elements of the N16 analysis. The calibrated SEDs provided in this work can be used to derive synthetic photometry in any passband from the UV to the IR, which in turn can be used to calibrate observations onto our photometric system tied directly to the three CALSPEC primary standards. The internal precision of our network is better than $5 \mathrm{mmag}$ in the optical, and the accuracy to which our photometric system is tied to CALSPEC is conservatively $4 \mathrm{mmag}$. This meets or exceeds the needs of most planned facilities.

In the course of our analysis, we identified and quantified the few systematic effects that may affect our program, the largest of which are how well our network is tied to the CALSPEC flux scale and a count rate nonlinearity exhibited by the IR channel of HST/WFC3. The biases in the inferred SEDs caused by these effects are small, and in a future analysis we expect to be able to mitigate these sources of systematic error further. Our ability to measure systematic effects at the few millimagnitude level reflect the precision of our programthese subtle signals would be swamped by other statistical systematic errors with a purely ground-based analysis. Our network of DA WDs is the most internally accurate with CALSPEC and precise spectrophotometric references with 16.5 mag $<V<19$ mag currently available. This is a strong claim, but one that we feel is validated by the suite of diagnostic tests performed in Section 8 .

Our network of standards can be of immediate benefit to several ongoing surveys. The SDSS and the Supernova Legacy Survey (SNLS) AB offsets (Betoule et al. 2013) were determined from the three primary CALSPEC standards, observed with a different facility and transformed to the survey telescope using comparisons of observed photometry and synthetic stellar spectral libraries. S15 determined additional corrections to place these magnitudes on the same system as the Pan-STARRS PS1 magnitudes, with its own AB offsets determined using seven CALSPEC standards. Each of these steps introduces potential systematic errors and is only necessary because the existing CALSPEC standards are too bright and the primary standards are inaccessible from the south. Our network of faint northern and equatorial DA WDs already addresses both these limitations and simplifies the calibration procedure to (i) observe DA WD stars, (ii) determine synthetic magnitudes of DA WDs from the SEDs published with this work, and (iii) determine the difference.

\subsection{Future Work}

The simplest expansion on this work is to expand the data set. Our Cycle 25 HST/WFC3 observations of southern DA WDs have been executed, and we are refining the data reduction and analysis drawing on lessons learned from C19 and this work. Our temporal monitoring of these southern standards is almost complete. Our next analysis will use this expanded data set. With the addition of any southern DA WDs that meet the stringent criteria in this work, we will have established our all-sky network. While HST's lifetime is limited, our network will extend its legacy of precise calibration well into the future. Moreover, the methodology developed in this work can be used to expand this network further in the future. In particular, there is no conceptual difficulty in establishing faint DA WDs as spectrophotometric standards tied to CALSPEC located within LSST DeepDrilling Fields (DDF) or WFIRST SN Survey fields.

Mitigating the sources of systematic error that affect our SEDs beyond the level accomplished in this work requires more complex changes to our methodology. Our present analysis treats each WD separately, fitting a single spectrum and the photometry for each. A minimal extension would be to infer results incorporating multiple spectra for each object where available. This would allow us to infer the SEDs of each 
star, including the primary standards, coherently from all available high-S/N data.

As in our previous analysis, this work establishes DA WD models as good differential predictors of measured flux ratios. The flux scale is itself set by the CALSPEC SEDs of the three primary standards, and there is no way to determine whether this flux scale is accurately tied to the AB system within our framework. The only way to avoid tying our network to the CALSPEC flux scale is to establish a single common flux scale for the CALSPEC standards and our faint DA WDs. Our analysis in this work treats the DA WDs hierarchically when inferring their apparent magnitudes, but individually in order to infer the parameters that describe their SED. The advantage to this two-step approach is that the model in Section 4.2 is completely independent of the intrinsic and extrinsic DA WD parameters. While this simple separable framework is conceptually appealing, the apparent magnitudes are directly tied to the CALSPEC SEDs of the three primary standards, which may have their own systematic errors.

It is possible to construct a single hierarchical model to describe the instrumental measurements and spectroscopy of all the DA WDs directly and the CALSPEC standards, without the intermediate hierarchical model to infer the apparent magnitudes. This model could be simultaneously conditioned on the three primary standards and our DA WDs to establish a single photometric system from $V \sim 9$ to $19 \mathrm{mag}$, incorporating measurements of laboratory or satellite-born flux standards, with the model atmosphere grid to calibrate the absolute flux. This model would be significantly more complex than the analysis presented in this work-instead of a 10-dimensional posterior distribution, we would be constraining $N_{\mathrm{s}} \times\left(5+5 \cdot N_{s}^{\lambda}\right)+$ $3 \cdot N_{\mathrm{PB}}$ parameters at once, where $N_{\mathrm{s}}$ is the number of objects, $N_{s}^{\lambda}$ is the number of spectra per object, and $N_{\mathrm{PB}}$ is the number of independent passbands. Even with conservative assumptions about the number of DA WDs from our Cycle 25 program that make good spectrophotometric standards, this would be a 350dimensional problem. Inference of the parameters of this model would require the development of bespoke sampling algorithms and significant computational resources.

There are few astrophysical sources that are as simple to model as DA WDs and capable of delivering the level of photometric accuracy achieved in our analysis. While our focus is on the accuracy of the fluxes of our SED models, various studies have focused on testing the absolute accuracy of inferred WD intrinsic parameters such as temperature, surface gravity, and mass using a variety of techniques, including determinations from eclipsing binaries (Parsons et al. 2017), gravitational lensing (Sahu et al. 2017), dynamical studies (Bond et al. 2017) and gravitational redshifts (Joyce et al. 2018b), and comparisons to other space-based missions such as FUSE (Joyce et al. 2018a) and Gaia (Tremblay et al. 2019). These efforts may lead to refinements in the existing DA WD model atmosphere grid, which in turn can be propagated to our inferred SEDs, yielding higher photometric accuracy.

The colors of our DA WD stars are much bluer than the main sequence, and we encourage their use to determine relative zero-points, rather than to determine color transformationsthe latter would require red standards within the range covered by the main sequence. We feel that this is largely a drawback of how transformation equations are parameterized, as they conflate establishing zero-points with determining color terms between different surveys' photometric systems. The choice of which color to use to parameterize these transformations, and indeed the choice to restrict the transformation equations to a single color, is entirely arbitrary. There are more sophisticated statistical methods of establishing the latter that model the nonlinear shape of the stellar locus (e.g., High et al. 2009; Kelly et al. 2014), rather than simple linear equations. We are examining the feasibility of combining such stellar locus regression techniques with the methodology in this work. It is valuable to have calibrated red stars within a few arcminutes of our DA WDs to verify the transformation equations between surveys, irrespective of how the transformations are derived. For this reason, we obtained HST/ACS parallel observations in F475W and F775W to provide field stars that can be tied to our WDs with color information. The properties of these stars will be presented in a future work.

The most complex extension of our analysis would be incorporating photometry from major surveys to constrain the shape of the entire stellar locus, solving for relative offsets within each survey from overlapping images and establishing the absolute zero-points using our network of DA WDs. Such a model would combine the Übercal (Padmanabhan et al. 2008) method, to establish uniform internal photometry, with stellar locus regression, to determine relative offsets between surveys as a function of color, and would use our DA WD stars and laboratory references to set the absolute flux scale. This would be an invaluable all-sky photometric catalog and enable numerous new studies.

G.N. is supported by the Lasker Fellowship at the Space Telescope Science Institute. E.O. was also partially supported by the NSF through grants AST-1313006 and AST-1815767.

Based on observations made with the NASA/ESA Hubble Space Telescope, obtained from the Mikulski Archive for Space Telescopes (MAST) at the Space Telescope Science Institute. STScI is operated by the Association of Universities for Research in Astronomy, Inc., under NASA contract NAS 5-26555. These observations are associated with programs GO-12967 (http://www.stsci.edu/cgi-bin/ get-proposal-info?id=12967\&observatory $=\mathrm{HST}$ ) and GO13711 (http://www.stsci.edu/cgi-bin/get-proposal-info? $\mathrm{id}=13711$ \&observatory=HST). We thank the staff at STScI, and in particular our program coordinators, Tricia Royle and Miranda Link, for their assistance with implementing our program.

Observations reported here were obtained at the MMT Observatory, a joint facility of the University of Arizona and the Smithsonian Institution. Based on observations obtained at the Gemini Observatory, which is operated by the Association of Universities for Research in Astronomy, Inc., under a cooperative agreement with the NSF on behalf of the Gemini partnership: the National Science Foundation (United States), the National Research Council (Canada), CONICYT (Chile), Ministerio de Ciencia, Tecnología e Innovación Productiva (Argentina), and Ministério da Ciência, Tecnologia e Inovação (Brazil). Based on observations obtained at the Southern Astrophysical Research (SOAR) telescope, which is a joint project of the Ministério da Ciência, Tecnologia, Inovações e Comunicações (MCTIC) do Brasil, the U.S. National Optical Astronomy Observatory (NOAO), the University of North Carolina at Chapel Hill (UNC), and Michigan State University (MSU). This work makes use of observations from the LCOGT 
network, the Pan-STARRS PS1 Survey, and the Sloan Digital Sky Survey (SDSS).

The Pan-STARRS1 Surveys (PS1) have been made possible through contributions of the Institute for Astronomy, the University of Hawaii, the Pan-STARRS Project Office, the Max-Planck Society and its participating institutes, the Max Planck Institute for Astronomy, Heidelberg, and the Max Planck Institute for Extraterrestrial Physics, Garching, Johns Hopkins University, Durham University, the University of Edinburgh, Queen's University Belfast, the Harvard-Smithsonian Center for Astrophysics, the Las Cumbres Observatory Global Telescope Network Incorporated, the National Central University of Taiwan, the Space Telescope Science Institute, the National Aeronautics and Space Administration under grant No. NNX08AR22G issued through the Planetary Science Division of the NASA Science Mission Directorate, the National Science Foundation under grant No. AST-1238877, the University of Maryland, and Eotvos Lorand University (ELTE).

Funding for SDSS-III has been provided by the Alfred P. Sloan Foundation, the Participating Institutions, the National Science Foundation, and the U.S. Department of Energy Office of Science. The SDSS-III website is http://www.sdss3.org/.

SDSS-III is managed by the Astrophysical Research Consortium for the Participating Institutions of the SDSS-III Collaboration, including the University of Arizona, the Brazilian Participation Group, Brookhaven National Laboratory, Carnegie Mellon University, University of Florida, the French Participation Group, the German Participation Group, Harvard University, the Instituto de Astrofisica de Canarias, the Michigan State/Notre Dame/JINA Participation Group, Johns Hopkins University, Lawrence Berkeley National Laboratory, Max Planck Institute for Astrophysics, Max Planck Institute for Extraterrestrial Physics, New Mexico State University, New York University, Ohio State University, Pennsylvania State University, University of Portsmouth, Princeton University, the Spanish Participation Group, University of Tokyo, University of Utah, Vanderbilt University, University of Virginia, University of Washington, and Yale University.

This work has made use of data from the European Space Agency (ESA) mission Gaia (https://www.cosmos.esa.int/ gaia), processed by the Gaia Data Processing and Analysis Consortium (DPAC, https://www.cosmos.esa.int/web/gaia/ dpac/consortium). Funding for the DPAC has been provided by national institutions, in particular the institutions participating in the Gaia Multilateral Agreement.

The analyses in this paper were run on the Odyssey cluster supported by the FAS Division of Science, Research Computing Group at Harvard University.

We are grateful to Adam Riess for insightful discussions about binarity in WD systems and the CRNL, as well as serving as contact scientist for our Cycle 22 program. Our analysis benefited from observations obtained by the Foundation Survey (P.I.s: Armin Rest, Daniel Scolnic, Ryan Foley) with the Pan-STARRS PS1 telescope. We thank the Foundation team for obtaining these images, and David Jones in particular for reducing the data and providing us with catalogs. We thank Daniel Foreman-Mackay for his thoughts on Gaussian process kernel design and producing valuable opensource tools, Eddie Schlafly for providing background on the dust maps, Daniel Scolnic for discussions on Supercal and for providing PS1 passband response functions, and members of the Dark Energy Survey calibration team, in particular Deborah Guellidge and Douglas Tucker, for testing our WDmodel code and providing feedback. This work would not be possible without the diligent efforts of IT staff at STScI, NOAO, and Harvard University, and we express our thanks to Michael Peralta (NOAO) and Eric Winter (STScI) for their tireless assistance. G.N. is grateful to Helmut Abt for several useful and entertaining discussions on the history of photometric calibration and providing him with M\&Ms during long remote observing runs.

This work made extensive use of several open-source packages, including the following:

Software: astropy (Astropy Collaboration et al. 2013; PriceWhelan et al. 2018), celerite (Foreman-Mackey et al. 2017), emcee (Foreman-Mackey et al. 2013), extinction, iminuit (iminuit team 2017), matplotlib (Hunter 2007), numpy (Oliphant 2015), pymc3 (Salvatier et al. 2016), pysynphot (STScI development Team 2013), scipy (Jones et al. 2001), and the conda package manager.

Facilities: HST (WFC3), Gemini:North (GMOS), Gemini: South (GMOS), MMT (Blue Channel), SOAR, Magellan: Baade (IMACS), LCOGT.

\section{ORCID iDs}

Gautham Narayan (ํ) https://orcid.org/0000-0001-6022-0484 Thomas Matheson (ib https://orcid.org/0000-0001-6685-0479 Abhijit Saha (1) https://orcid.org/0000-0002-6839-4881 Tim Axelrod (i) https://orcid.org/0000-0002-5722-7199 Annalisa Calamida (1) https://orcid.org/0000-0002-0882-7702 Kaisey S. Mandel iㅣ https://orcid.org/0000-0001-9846-4417 Ralph C. Bohlin (10 https://orcid.org/0000-0001-9806-0551 Susana Deustua (iD https://orcid.org/0000-0003-2823-360X Christopher W. Stubbs (1) https://orcid.org/0000-00030347-1724

Elena Sabbi iㅏ https://orcid.org/0000-0003-2954-7643

\section{References}

Alam, S., Albareti, F. D., Allende Prieto, C., et al. 2015, ApJS, 219, 12 Allende Prieto, C., Hubeny, I., \& Smith, J. A. 2009, MNRAS, 396, 759 Astropy Collaboration, Robitaille, T. P., Tollerud, E. J., et al. 2013, A\&A, 558, A33

Bajaj, V., \& Khandrika, H. 2017, Comparing Aperture Photometry Software Packages Tech. Rep. WFC3 2017-10 (Baltimore, MD: STSci)

Barbary, K. 2016, extinction v0.3.0, Zenodo, doi:10.5281/zenodo.804967

Bergeron, P., Saffer, R. A., \& Liebert, J. 1992a, ApJ, 394, 228

Bergeron, P., Wesemael, F., \& Fontaine, G. 1992b, ApJ, 387, 288

Bertin, E., \& Arnouts, S. 1996, A\&AS, 117, 393

Betoule, M., Marriner, J., Regnault, N., et al. 2013, A\&A, 552, A124

Bohlin, R. C. 2014, AJ, 147, 127

Bohlin, R. C., Gordon, K. D., \& Tremblay, P. E. 2014, PASP, 126, 711

Bohlin, R. C., Riess, A., \& de Jong, R. 2006, NICMOS Count Rate Dependent Non-linearity in G096 and G141 Tech. Rep. NICMOS 2006-002 (Baltimore, MD: STSci)

Bond, H. E., Bergeron, P., \& Bédard, A. 2017, ApJ, 848, 16

Bradley, L., Sipocz, B., Robitaille, T., et al. 2017, astropy/photutils v0.4, Zenodo, doi:10.5281/zenodo.1039309

Brinkworth, C. S., Burleigh, M. R., Lawrie, K., Marsh, T. R., \& Knigge, C. 2013, ApJ, 773, 47

Calamida, A., Matheson, T., Saha, A., et al. 2019, ApJ, 872, 199

Casagrande, L., \& VandenBerg, D. A. 2018, MNRAS, 479, L102

Czekala, I., Andrews, S. M., Mandel, K. S., Hogg, D. W., \& Green, G. M. 2015, ApJ, 812, 128

de Jong, R. S. 2006, Correcting the NICMOS Count-rate Dependent Nonlinearity, Tech. Rep. NICMOS 2006-003 (Baltimore, MD: STSci)

de Jong, R. S., Bergeron, E., Riess, A., \& Bohlin, R. 2006, NICMOS Countrate Dependent Nonlinearity Tests Using Flatfield Lamps Tech. Rep. NICMOS 2006-001 (Baltimore, MD: STSci) 
Deustua, S. E. 2016, Updated WFC3/UVIS Chip Dependent SYNPHOT/ PYSYNPHOT Files, Tech. Rep. WFC3 2016-07 (Baltimore, MD: STSci)

Deustua, S. E., Mack, J., Bajaj, V., \& Khandrika, H. 2017, WFC3/UVIS Updated 2017 Chip-Dependent Inverse Sensitivity Values, Tech. Rep. WFC3 2017-14 (Baltimore, MD: STSci), http://www.stsci.edu/hst/wfc3/ documents/ISRs/WFC3-2017-14.pdf

Deustua, S. E., Mack, J., \& Bowers, A. S. 2016, UVIS 2.0 Chip-dependent Inverse Sensitivity Values, Tech. Rep. WFC3 2016-03 (Baltimore, MD: STSci), http://www.stsci.edu/hst/wfc3/documents/ISRs/WFC3-2016-03.pdf

Eisenstein, D. J., Liebert, J., Harris, H. C., et al. 2006, ApJS, 167, 40

Evans, D. W., Riello, M., De Angeli, F., et al. 2018, A\&A, 616, A4

Farihi, J., Gänsicke, B. T., Steele, P. R., et al. 2012, MNRAS, 421, 1635

Fitzpatrick, E. L. 1999, PASP, 111, 63

Fitzpatrick, E. L., \& Massa, D. 1990, ApJS, 72, 163

Flewelling, H. A., Magnier, E. A., Chambers, K. C., et al. 2016, arXiv:1612. 05243

Foreman-Mackey, D., Agol, E., Angus, R., \& Ambikasaran, S. 2017, AJ, 154,220

Foreman-Mackey, D., Hogg, D. W., Lang, D., \& Goodman, J. 2013, PASP, 125,306

Fukugita, M., Ichikawa, T., Gunn, J. E., et al. 1996, AJ, 111, 1748

Gelman, A. 2006, BayAn, 1, 515

Gelman, A., Carlin, J. B., Stern, H. S., \& Rubin, D. B. 2004, Bayesian Data Analysis (2nd ed.; Boca Raton, FL: Chapman and Hall/CRC Press)

Gelman, A., \& Rubin, D. B. 1992, StaSc, 7, 457

Gentile Fusillo, N. P., Tremblay, P.-E., Gänsicke, B. T., et al. 2019, MNRAS, 482, 4570

Gianninas, A., Dufour, P., Kilic, M., et al. 2014, ApJ, 794, 35

Hartig, G. F. 2008, WFC3 UVIS Shutter Vibration-Induced Image Blur, Tech. Rep. WFC3 2008-44 (Baltimore, MD: STSci)

Hermes, J. J., Gänsicke, B. T., Gentile Fusillo, N. P., et al. 2017, MNRAS, 468, 1946

High, F. W., Stubbs, C. W., Rest, A., Stalder, B., \& Challis, P. 2009, AJ, 138, 110

Hobbs, L. M. 1984, ApJ, 280, 132

Hogg, D. W., Bovy, J., \& Lang, D. 2010, arXiv:1008.4686

Homan, M. D., \& Gelman, A. 2014, J. Mach. Learn. Res., 15, 1593

Hook, I. M., Jørgensen, I., Allington-Smith, J. R., et al. 2004, PASP, 116, 425

Horne, K. 1986, PASP, 98, 609

Hubeny, I., Hummer, D. G., \& Lanz, T. 1994, A\&A, 282, 151

Hubeny, I., \& Lanz, T. 1995, ApJ, 439, 875

Hubeny, I., \& Lanz, T. 2017, arXiv:1706.01859

Hunter, J. D. 2007, CSE, 9, 90

iminuit team 2017, iminuit-A Python interface to Minuit, https://github. com/iminuit/iminuit

Jones, E., Oliphant, T., Peterson, P., et al. 2001, SciPy: Open source scientific tools for Python

Joyce, S. R. G., Barstow, M. A., Casewell, S. L., et al. 2018a, MNRAS, 479, 1612

Joyce, S. R. G., Barstow, M. A., Holberg, J. B., et al. 2018b, MNRAS, 481, 2361

Kelly, P. L., von der Linden, A., Applegate, D. E., et al. 2014, MNRAS, 439, 28

Kleinman, S. J., Kepler, S. O., Koester, D., et al. 2013, ApJS, 204, 5

Koester, D., Gänsicke, B. T., \& Farihi, J. 2014, A\&A, 566, A34

Koornneef, J., Bohlin, R., Buser, R., Horne, K., \& Turnshek, D. 1986, HiA, 7, 833

Landolt, A. U. 1992, AJ, 104, 340

Leonard, D. C., \& Filippenko, A. V. 2001, PASP, 113, 920

Levenhagen, R. S., Diaz, M. P., Coelho, P. R. T., \& Hubeny, I. 2017, ApJS, 231, 1

Liebert, J., Bergeron, P., \& Holberg, J. B. 2003, AJ, 125, 348
Magnier, E. A., Schlafly, E., Finkbeiner, D., et al. 2013, ApJS, 205, 20

Magnier, E. A., Schlafly, E. F., Finkbeiner, D. P., et al. 2016, arXiv:1612. 05242

Megessier, C. 1995, A\&A, 296, 771

Narayan, G., Axelrod, T., Holberg, J. B., et al. 2016, ApJ, 822, 67

O’Donnell, J. E. 1994, ApJ, 422, 158

Oke, J. B. 1990, AJ, 99, 1621

Oke, J. B., \& Gunn, J. E. 1983, ApJ, 266, 713

Oliphant, T. E. 2015, Guide to NumPy (2nd ed.; Seattle, WA: CreateSpace Independent Publishing Platform)

Padmanabhan, N., Schlegel, D. J., Finkbeiner, D. P., et al. 2008, ApJ, 674, 1217

Parsons, S. G., Gänsicke, B. T., Marsh, T. R., et al. 2017, MNRAS, 470, 4473

Poznanski, D., Prochaska, J. X., \& Bloom, J. S. 2012, MNRAS, 426, 1465

Price-Whelan, A. M., Sipőcz, B. M., Günther, H. M., et al. 2018, AJ, 156, 123

Rasmussen, C. E., \& Williams, C. K. I. 2005, Gaussian Processes for Machine Learning (Cambridge, MA: MIT Press)

Rauch, T. 2016, in ASP Conf. Ser. 503, The Science of Calibration, ed. S. Deustua et al. (San Francisco, CA: ASP), 193

Rauch, T., Werner, K., Bohlin, R., \& Kruk, J. W. 2013, A\&A, 560, A106

Rest, A., Stubbs, C., Becker, A. C., et al. 2005, ApJ, 634, 1103

Riello, M., De Angeli, F., Evans, D. W., et al. 2018, A\&A, 616, A3

Riess, A. G. 2010, First On-orbit Measurements of the WFC3-IR Count-rate Non-linearity, Tech. Rep. WFC3 2010-07 (Baltimore, MD: STSci)

Riess, A. G. 2011, An Independent Determination of WFC3-IR Zeropoints and Count Rate Non-linearity from 2MASS Asterisms, Tech. Rep. WFC3 201115 (Baltimore, MD: STSci)

Riess, A. G., \& Petro, L. 2010, Boosting Count-rates with Earth Limb Light and the WFC3/IR Count-rate Non-linearity, Tech. Rep. WFC3 2010-15 (Baltimore, MD: STSci)

Sahu, K., Baggett, S., \& MacKenty, J. 2014, Use of the Shutter Blade Side for UVIS Short Exposures, Tech. Rep. WFC3 2014-009 (Baltimore, MD: STSci)

Sahu, K. C., Anderson, J., Casertano, S., et al. 2017, Sci, 356, 1046

Salvatier, J., Wiecki, T. V., \& Fonnesbeck, C. 2016, PeerJ Comp. Sci., 2, e55

Schlafly, E. F., \& Finkbeiner, D. P. 2011, ApJ, 737, 103

Schlafly, E. F., Finkbeiner, D. P., Jurić, M., et al. 2012, ApJ, 756, 158

Schlafly, E. F., Meisner, A. M., Stutz, A. M., et al. 2016, ApJ, 821, 78

Scolnic, D., Casertano, S., Riess, A., et al. 2015, ApJ, 815, 117

Scolnic, D., Rest, A., Riess, A., et al. 2014, ApJ, 795, 45

Shanahan, C. E., Gosmeyer, C. M., \& Baggett, S. 2017, 2017 Update on the WFC3/UVIS Stability and Contamination Monitor, Tech. Rep. WFC3 2017 15 (Baltimore, MD: STSci), http://www.stsci.edu/hst/wfc3/documents/ ISRs/WFC3-2017-15.pdf

Silvestri, N. M., Hawley, S. L., West, A. A., et al. 2006, AJ, 131, 1674

Stetson, P. B. 1987, PASP, 99, 191

STScI Development Team 2013, pysynphot: Synthetic photometry software package, Astrophysics Source Code Library, ascl:1303.023

Stubbs, C. W., \& Brown, Y. J. 2015, MPLA, 30, 1530030

Stubbs, C. W., \& Tonry, J. L. 2006, ApJ, 646, 1436

Toonen, S., Hollands, M., Gänsicke, B. T., \& Boekholt, T. 2017, A\&A, 602, A16

Tremblay, P.-E., \& Bergeron, P. 2009, ApJ, 696, 1755

Tremblay, P.-E., Cukanovaite, E., Gentile Fusillo, N. P., Cunningham, T., \& Hollands, M. A. 2019, MNRAS, 482, 5222

Welch, D. L., \& Stetson, P. B. 1993, AJ, 105, 1813

Winget, D., \& Kepler, S. 2008, ARA\&A, 46, 157

Wood-Vasey, W. M., Miknaitis, G., Stubbs, C. W., et al. 2007, ApJ, 666, 694 